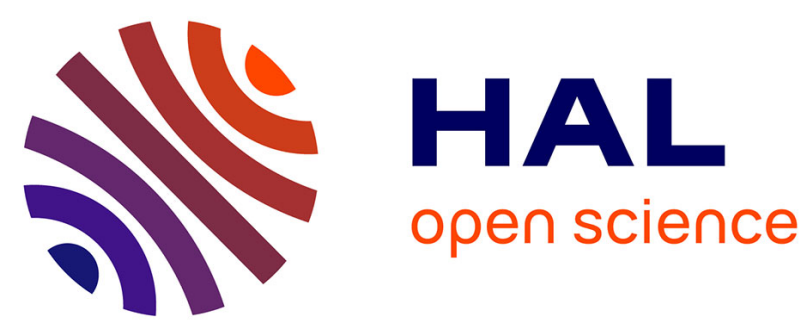

\title{
Generalized Robin-Neumann explicit coupling schemes for incompressible fluid-structure interaction: stability analysis and numerics
}

Miguel Angel Fernández, Jimmy Mullaert, Marina Vidrascu

\section{- To cite this version:}

Miguel Angel Fernández, Jimmy Mullaert, Marina Vidrascu. Generalized Robin-Neumann explicit coupling schemes for incompressible fluid-structure interaction: stability analysis and numerics. International Journal for Numerical Methods in Engineering, 2015, 101 (3), pp.199-229. 10.1002/nme.4785 . hal-00875819

HAL Id: hal-00875819

https://hal.inria.fr/hal-00875819

Submitted on 24 Oct 2013

HAL is a multi-disciplinary open access archive for the deposit and dissemination of scientific research documents, whether they are published or not. The documents may come from teaching and research institutions in France or abroad, or from public or private research centers.
L'archive ouverte pluridisciplinaire HAL, est destinée au dépôt et à la diffusion de documents scientifiques de niveau recherche, publiés ou non, émanant des établissements d'enseignement et de recherche français ou étrangers, des laboratoires publics ou privés. 
Generalized

Robin-Neumann explicit coupling schemes for incompressible fluid-structure interaction: stability analysis and numerics

Miguel A. Fernández, Jimmy Mullaert, Marina Vidrascu

RESEARCH REPORT

$\mathbf{N}^{\circ} 8384$

October 2013

Project-Team Reo 



\title{
Givat
}

\section{Generalized Robin-Neumann explicit coupling schemes for incompressible fluid-structure interaction: stability analysis and numerics}

\author{
Miguel A. Fernández ${ }^{* \dagger}$, Jimmy Mullaert*†, Marina Vidrascu*† \\ Project-Team Reo \\ Research Report $n^{\circ} 8384$ - October 2013 - 35 pages
}

\begin{abstract}
We introduce a new class of explicit coupling schemes for the numerical solution of fluid-structure interaction problems involving a viscous incompressible fluid and an elastic structure. These methods generalize the arguments reported in $[13,10]$ to the case of the coupling with thick-walled structures. The basic idea lies in the derivation of an intrinsic interface Robin consistency at the space semi-discrete level, using a lumped-mass approximation in the structure. The fluid-solid splitting is then performed through appropriate extrapolations of the solid velocity and stress on the interface. Based on these methods, a new, parameter-free, Robin-Neumann iterative procedure is also proposed for the partitioned solution of implicit coupling. A priori energy estimates, guaranteeing the stability of the schemes and the convergence of the iterative procedure, are established. The accuracy and robustness of the methods are illustrated in several numerical examples.
\end{abstract}

Key-words: fluid-structure interaction, incompressible fluid, thick-walled structure, explicit coupling scheme, Robin-Neumann methods.

This work was supported by the French National Research Agency (ANR) through the EXIFSI project (ANR-12-JS01-0004).

* Inria, REO project-team, Rocquencourt - B.P. 105, F-78153 Le Chesnay cedex, France

$\dagger$ UPMC Univ Paris VI, REO project-team, UMR 7958 LJLL, F-75005 Paris, France

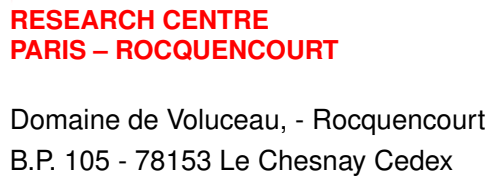




\section{Schémas Robin-Neumann explicites pour le couplage d'un fluide incompressible avec une structure mince}

Résumé : Cet article présente une nouvelle famille de schémas explicites pour l'approximation numérique de problèmes d'interaction fluide-structure faisant intervenir un fluide visqueux incompressible et une structure élastique. Ces méthodes étendent les arguments introduits dans $[13,10]$ au cas du couplage avec une structure épaisse. L'idée principale est d'introduire, à l'aide d'une condensation de la matrice de masse solide, une condition de couplage consistante de type Robin dans la formulation semi-discrète en espace. L'extrapolation de la vitesse solide ainsi que des efforts à l'interface permet alors d'établir un schéma explicite. Cette même méthode fournit également une procédure itérative pour la résolution du schéma de couplage implicite. Des estimations d'énergie a priori sont démontrées et garantissent la stabilité des schémas explicites, mais aussi la convergence de la méthode itérative. La précision et la robustesse de ces méthodes sont évaluées numériquement dans plusieurs exemples.

Mots-clés : interaction fluide-structure, fluide incompressible, structure épaisse, schéma de couplage explicite, schéma Robin-Neumann. 


\section{Introduction}

Mathematical problems describing the coupling of an elastic structure with an incompressible fluid, appear in a variety of engineering fields, from the aeroelasticity of bridge decks and parachutes, to naval hydrodynamics and the biomechanics of air and blood flow. Over the last decade, the development of efficient numerical methods for these type of problems has been an extremely active field of research (see, e.g., [9, 20] for recent reviews).

This is due, in particular, to the fact that the coupling is very stiff. So called explicit coupling (or loosely coupled, see [27, 28, 8]) schemes, that only involve the solution of the fluid and of the structure once per time-step, are known to be unconditionally unstable for standard DirichletNeumann strategies whenever the amount of added-mass in the system is strong (see, e.g., [6, 16]). In view of this, much research effort has gone into the design of robust solvers for the solution of the more computationally onerous implicit and semi-implicit coupling paradigms (see, e.g., $[11,29,2,22,1,18,17,7,26,24,25])$.

Stability in explicit coupling requires a different treatment of the interface coupling conditions. In [4], added-mass free stability is achieved through a specific Robin-Robin treatment of the coupling, derived from Nitsche's method, together with an interface pressure stabilization in time. The price to pay is the deterioration of the accuracy, which demands restrictive CFL constraints, unless enough correction iterations are performed (see [4, 5]). In the case of the coupling with a thin-walled structure, both added-mass free stability and optimal (first-order) time accuracy are obtained with the explicit Robin-Neumann schemes proposed in [13, 10]. The fundamental ingredient in the derivation of these schemes is the interface Robin consistency of the continuous problem, which is intimately related to thin-walled character of the solid model.

In this paper, we propose an extension (the first, to the best of our knowledge) of the explicit coupling schemes reported in $[13,10]$ to the case of the coupling with thick-walled structures: linear and non-linear (possibly damped) elasticity. We show that an intrinsic (parameter free) interface Robin consistency can be recovered at the space semi-discrete level, using a lumpedmass approximation in the structure. Instead of the usual identity operator (as in [13, 10]), the generalized Robin condition involves a new interface operator which consistently accounts for the solid inertial effects within the fluid. The fluid-solid splitting is hence performed through appropriate extrapolations of the solid velocity and stress on the interface. A priori energy estimates, guaranteeing (added-mass) free stability, are derived for all the extrapolations considered.

The second contribution of this work deals with the partitioned solution of implicit coupling. In fact, the proposed explicit coupling schemes can be interpreted as a single iteration (with appropriate initializations) of a new Robin-Neumann iterative method. Unlike traditional Robin based procedures (see, e.g., [1]), these iterations are parameter free. Using energy arguments, we demonstrate the (added-mass free) convergence of this iterative procedure towards the implicit coupling solution. To the best of our knowledge, the error estimate proposed is the first which yields convergence of a Robin-Neumann procedure in the framework of the coupling with a thick-walled structure (linear viscoelasticity).

Several numerical experiments, based on different linear and non-linear fluid-structure interaction examples from the literature, illustrate the accuracy and robustness of the proposed schemes.

The paper is organized as follows. In Section 2, we present the linear continuous setting which serves as model coupled problem. In Section 3, we introduce the generalized Robin-Neumann explicit coupling schemes. The iterative procedure for the partitioned solution of implicit coupling is also presented. Section 4 is devoted to the numerical analysis of the methods. In Section 5 , the coupling schemes are formulated in a fully non-linear framework. The numerical experiments are reported in Section 6. Finally, Section 7 summarizes the conclusions. 
Some preliminary results of this work have been announced, without proof, in [12].

\section{A linear model problem}

In order to ease the presentation, we first consider a low Reynolds regime and assume that the interface undergoes infinitesimal displacements. The fluid is described by the Stokes equations, in a fixed domain $\Omega^{\mathrm{f}} \subset \mathbb{R}^{d}(d=2,3)$, and the structure by the linear (possibly damped) elasticity equations, in the solid domain $\Omega^{\mathrm{s}} \subset \mathbb{R}^{d}$. We denote by $\Sigma \stackrel{\text { def }}{=} \partial \Omega^{\mathrm{s}} \cap \partial \Omega^{\mathrm{f}}$ the fluid-structure interface and $\partial \Omega^{\mathrm{f}}=\Gamma \cup \Sigma$ and $\partial \Omega^{\mathrm{s}}=\Gamma^{\mathrm{d}} \cup \Gamma^{\mathrm{n}} \cup \Sigma$ are given partitions of the fluid and solid boundaries, respectively (see Figure 1). The linear coupled problem reads as follows: Find the

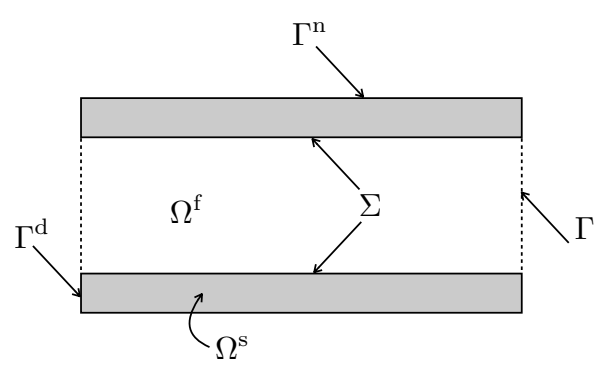

Figure 1: Geometrical description.

fluid velocity $\boldsymbol{u}: \Omega^{\mathrm{f}} \times \mathbb{R}^{+} \rightarrow \mathbb{R}^{d}$, the fluid pressure $p: \Omega^{\mathrm{f}} \times \mathbb{R}^{+} \rightarrow \mathbb{R}$, the structure displacement $\boldsymbol{d}: \Omega^{\mathrm{s}} \times \mathbb{R}^{+} \rightarrow \mathbb{R}^{d}$ and the structure velocity $\dot{\boldsymbol{d}}: \Omega^{\mathrm{s}} \times \mathbb{R}^{+} \rightarrow \mathbb{R}^{d}$ such that

$$
\begin{aligned}
& \left\{\begin{array}{rcc}
\rho^{\mathrm{f}} \partial_{t} \boldsymbol{u}-\operatorname{div} \boldsymbol{\sigma}^{\mathrm{f}}(\boldsymbol{u}, p)=\mathbf{0} & \text { in } \quad \Omega^{\mathrm{f}}, \\
\operatorname{div} \boldsymbol{u}=0 & \text { in } \quad \Omega^{\mathrm{f}}, \\
\boldsymbol{\sigma}^{\mathrm{f}}(\boldsymbol{u}, p) \boldsymbol{n}^{\mathrm{f}}=\boldsymbol{f}^{\Gamma} & \text { on } \quad \Gamma,
\end{array}\right. \\
& \left\{\begin{array}{rcc}
\rho^{\mathrm{s}} \partial_{t} \dot{\boldsymbol{d}}+\alpha \rho^{\mathrm{s}} \dot{\boldsymbol{d}}-\boldsymbol{d i v} \boldsymbol{\sigma}^{\mathrm{s}}(\boldsymbol{d}, \dot{\boldsymbol{d}})+c_{0} \boldsymbol{d}=\mathbf{0} & \text { in } \quad \Omega^{\mathrm{s}}, \\
\dot{\boldsymbol{d}}=\partial_{t} \boldsymbol{d} & \text { in } \quad \Omega^{\mathrm{s}}, \\
\boldsymbol{d}=\mathbf{0}, \beta \dot{\boldsymbol{d}}=\mathbf{0} & \text { on } \quad \Gamma^{\mathrm{d}}, \\
\boldsymbol{\sigma}^{\mathrm{s}}(\boldsymbol{d}, \dot{\boldsymbol{d}}) \boldsymbol{n}^{\mathrm{s}}=\mathbf{0} & \text { on } \quad \Gamma^{\mathrm{n}},
\end{array}\right.
\end{aligned}
$$

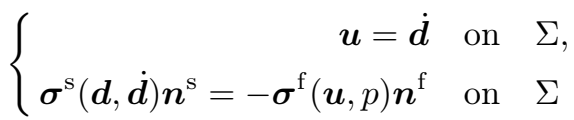

and satisfying the initial conditions $\boldsymbol{u}(0)=\boldsymbol{u}_{0}, \boldsymbol{d}(0)=\boldsymbol{d}_{0}$ and $\dot{\boldsymbol{d}}(0)=\boldsymbol{v}_{0}$. Here, $\rho^{\mathrm{f}}, \rho^{\mathrm{s}}>0$ stand for the fluid and solid densities, $\boldsymbol{f}^{\Gamma}$ for a given surface traction on $\Gamma$ and $\boldsymbol{n}^{\mathrm{f}}, \boldsymbol{n}^{\mathrm{s}}$ for the exterior unit normal vectors to the boundaries of $\Omega^{\mathrm{f}}$ and $\Omega^{\mathrm{s}}$, respectively. The fluid stress tensor $\boldsymbol{\sigma}^{\mathrm{f}}(\boldsymbol{u}, p)$ is given by

$$
\boldsymbol{\sigma}^{\mathrm{f}}(\boldsymbol{u}, p) \stackrel{\text { def }}{=}-p \boldsymbol{I}+2 \mu \boldsymbol{\epsilon}(\boldsymbol{u}), \quad \boldsymbol{\epsilon}(\boldsymbol{u}) \stackrel{\text { def }}{=} \frac{1}{2}\left(\boldsymbol{\nabla} \boldsymbol{u}+\boldsymbol{\nabla} \boldsymbol{u}^{\mathrm{T}}\right)
$$

where $\mu>0$ denotes the fluid dynamic viscosity. The solid stress tensor is given by

$$
\boldsymbol{\sigma}^{\mathrm{s}}(\boldsymbol{d}, \dot{\boldsymbol{d}}) \stackrel{\text { def }}{=} \boldsymbol{\sigma}(\boldsymbol{d})+\beta \boldsymbol{\sigma}(\dot{\boldsymbol{d}}), \quad \boldsymbol{\sigma}(\boldsymbol{d}) \stackrel{\text { def }}{=} 2 L_{1} \boldsymbol{\epsilon}(\boldsymbol{d})+L_{2}(\operatorname{div} \boldsymbol{d}) \boldsymbol{I}
$$


where $L_{1}, L_{2}>0$ stand for the Lamé constants of the structure. Therefore, the viscous effects in the structure are described in (2) by the term

$$
\alpha \rho^{\mathrm{s}} \dot{\boldsymbol{d}}-\beta \operatorname{div} \boldsymbol{\sigma}(\dot{\boldsymbol{d}}), \quad \alpha, \beta \geq 0,
$$

which corresponds to a Rayleigh modeling of the solid damping (see e.g., [21]). The zerothorder term $c_{0} \boldsymbol{d}$ in (2), with $c_{0} \geq 0$, represents the transversal membrane effects that appear in axisymmetric formulations.

In what follows, we will make use of the following functional spaces $\boldsymbol{V}^{\mathrm{f}} \stackrel{\text { def }}{=}\left[H^{1}\left(\Omega^{\mathrm{f}}\right)\right]^{d}, Q \stackrel{\text { def }}{=}$ $L^{2}\left(\Omega^{\mathrm{f}}\right), \boldsymbol{V}^{\mathrm{s}} \stackrel{\text { def }}{=}\left\{\boldsymbol{v}^{\mathrm{s}} \in\left[H^{1}\left(\Omega^{\mathrm{s}}\right)\right]^{d} /\left.\boldsymbol{v}^{\mathrm{s}}\right|_{\Gamma^{\mathrm{d}}}=\mathbf{0}\right\}, \boldsymbol{W} \stackrel{\text { def }}{=}\left\{\left(\boldsymbol{v}^{\mathrm{f}}, \boldsymbol{v}^{\mathrm{s}}\right) \in \boldsymbol{V}^{\mathrm{f}} \times \boldsymbol{V}^{\mathrm{s}} /\left.\boldsymbol{v}^{\mathrm{f}}\right|_{\Sigma}=\left.\boldsymbol{v}^{\mathrm{s}}\right|_{\Sigma}\right\}$ and the following bi-linear and linear forms

$$
\begin{aligned}
& a\left(\boldsymbol{u}, \boldsymbol{v}^{\mathrm{f}}\right) \stackrel{\text { def }}{=} 2 \mu\left(\boldsymbol{\epsilon}(\boldsymbol{u}), \boldsymbol{\epsilon}\left(\boldsymbol{v}^{\mathrm{f}}\right)\right)_{\Omega^{\mathrm{f}}}, \quad b\left(p, \boldsymbol{v}^{\mathrm{f}}\right) \stackrel{\text { def }}{=}-\left(p, \operatorname{div} \boldsymbol{v}^{\mathrm{f}}\right)_{\Omega^{\mathrm{f}}}, \quad l\left(\boldsymbol{v}^{\mathrm{f}}\right) \stackrel{\text { def }}{=}\left(\boldsymbol{f}^{\Gamma}, \boldsymbol{v}^{\mathrm{f}}\right)_{\Gamma}, \\
& a^{\mathrm{e}}\left(\boldsymbol{d}, \boldsymbol{v}^{\mathrm{s}}\right) \stackrel{\text { def }}{=}\left(\boldsymbol{\sigma}(\boldsymbol{d}), \boldsymbol{\epsilon}\left(\boldsymbol{v}^{\mathrm{s}}\right)\right)_{\Omega^{\mathrm{s}}}+c_{0}\left(\boldsymbol{d}, \boldsymbol{v}^{\mathrm{s}}\right)_{\Omega^{\mathrm{s}}}, \\
& a^{\mathrm{v}}\left(\dot{\boldsymbol{d}}, \boldsymbol{v}^{\mathrm{s}}\right) \stackrel{\text { def }}{=} \beta\left(\boldsymbol{\sigma}(\dot{\boldsymbol{d}}), \boldsymbol{\epsilon}\left(\boldsymbol{v}^{\mathrm{s}}\right)\right)_{\Omega^{\mathrm{s}}}+\alpha \rho^{\mathrm{s}}\left(\dot{\boldsymbol{d}}, \boldsymbol{v}^{\mathrm{s}}\right)_{\Omega^{\mathrm{s}}} .
\end{aligned}
$$

Here, the symbol $(\cdot, \cdot)_{\omega}$ stands for the standard inner-product of $L^{2}(\omega)$, for a given domain $\omega$ of $\mathbb{R}^{d}$ or $\mathbb{R}^{d-1}$.

The coupled problem (1)-(3) admits the following variational formulation: for $t>0$, find $(\boldsymbol{u}(t), \dot{\boldsymbol{d}}(t)) \in \boldsymbol{W}, p(t) \in Q$ and $\boldsymbol{d}(t) \in \boldsymbol{V}^{\mathrm{s}}$ such that $\dot{\boldsymbol{d}}=\partial_{t} \boldsymbol{d}$ and

$$
\begin{aligned}
& \rho^{\mathrm{f}}\left(\partial_{t} \boldsymbol{u}, \boldsymbol{v}^{\mathrm{f}}\right)_{\Omega^{\mathrm{f}}}+a\left(\boldsymbol{u}, \boldsymbol{v}^{\mathrm{f}}\right)+b\left(p, \boldsymbol{v}^{\mathrm{f}}\right)-b(q, \boldsymbol{u}) \\
& \quad+\rho^{\mathrm{s}}\left(\partial_{t} \dot{\boldsymbol{d}}, \boldsymbol{v}^{\mathrm{s}}\right)_{\Omega^{\mathrm{s}}}+a^{\mathrm{v}}\left(\dot{\boldsymbol{d}}, \boldsymbol{v}^{\mathrm{s}}\right)+a^{\mathrm{e}}\left(\boldsymbol{d}, \boldsymbol{v}^{\mathrm{s}}\right)=l\left(\boldsymbol{v}^{\mathrm{f}}\right)
\end{aligned}
$$

for all $\left(\boldsymbol{v}^{\mathrm{f}}, \boldsymbol{v}^{\mathrm{s}}\right) \in \boldsymbol{W}$ and $q \in Q$.

\section{Generalized Robin-Neumann methods}

This section is devoted to the numerical approximation of the coupled problem (4). The timemarching procedures proposed (Section 3.3 below) allow an uncoupled sequential computation of the fluid and solid discrete approximations (explicit coupling scheme). These methods can be viewed as a generalization to the coupling with thick-walled structures of the Robin-Neumann explicit schemes introduced in [13, 10].

A fundamental ingredient in the derivation of the schemes reported in $[13,10]$ is the interface Robin consistency of the continuous problem. Clearly, this property is not shared by the coupled problem (1)-(3), since it is intimately related to the thin-walled character of the structure. In Section 3.2, we show that an underlying interface Robin consistency can be recovered after discretization in space, using a lumped-mass approximation in the structure. This generalized notion of interface Robin consistency is the basis of the new explicit coupling schemes introduced in Section 3.3 and of the new iterative procedure proposed in Section 3.4.

\subsection{Space semi-discretization}

We consider a finite element approximations in space based on continuous piecewise affine functions. The corresponding finite element spaces are denoted by $\boldsymbol{V}_{h}^{\mathrm{f}} \subset \boldsymbol{V}^{\mathrm{f}}, Q_{h} \subset Q, \boldsymbol{V}_{h}^{\mathrm{s}} \subset \boldsymbol{V}^{\mathrm{s}}$, where the subscript $h>0$ indicates the level of spatial refinement. Since the fluid velocity/pressure pair $\boldsymbol{V}_{h}^{\mathrm{f}} / Q_{h}$ fails to satisfy the inf-sup condition, we consider, without loss of 
generality, a symmetric pressure stabilization method defined by a non-negative bilinear form, $s_{h}: Q_{h} \times Q_{h} \rightarrow \mathbb{R}$, entering the abstract framework introduced in [3]. Furthermore, we assume that the fluid and solid discretizations match at the interface, that is, $\boldsymbol{\Lambda}_{\Sigma, h} \stackrel{\text { def }}{=}\left\{\left.\boldsymbol{v}_{h}^{\mathrm{f}}\right|_{\Sigma} / \boldsymbol{v}_{h}^{\mathrm{f}} \in\right.$ $\left.\boldsymbol{V}_{h}^{\mathrm{f}}\right\}=\left\{\left.\boldsymbol{v}_{h}^{\mathrm{s}}\right|_{\Sigma} / \boldsymbol{v}_{h}^{\mathrm{s}} \in \boldsymbol{V}_{h}^{\mathrm{s}}\right\}$, and we set $\boldsymbol{W}_{h} \stackrel{\text { def }}{=}\left\{\left(\boldsymbol{v}_{h}^{\mathrm{f}}, \boldsymbol{v}_{h}^{\mathrm{s}}\right) \in \boldsymbol{V}_{h}^{\mathrm{f}} \times \boldsymbol{V}_{h}^{\mathrm{s}} /\left.\boldsymbol{v}_{h}^{\mathrm{f}}\right|_{\Sigma}=\left.\boldsymbol{v}_{h}^{\mathrm{s}}\right|_{\Sigma}\right\} \subset \boldsymbol{W}$, $\boldsymbol{V}_{\Sigma, h}^{\mathrm{f}} \stackrel{\text { def }}{=}\left\{\boldsymbol{v}_{h}^{\mathrm{f}} \in \boldsymbol{V}_{h}^{\mathrm{f}} /\left.\boldsymbol{v}_{h}^{\mathrm{f}}\right|_{\Sigma}=\mathbf{0}\right\}$ and $\boldsymbol{V}_{\Sigma, h}^{\mathrm{s}} \stackrel{\text { def }}{=}\left\{\boldsymbol{v}_{h}^{\mathrm{s}} \in \boldsymbol{V}_{h}^{\mathrm{s}} /\left.\boldsymbol{v}_{h}^{\mathrm{s}}\right|_{\Sigma}=\mathbf{0}\right\}$.

We denote by $(\cdot, \cdot)_{\Omega^{\mathrm{s}}, h}$ the lumped-mass approximation of the inner-product $(\cdot, \cdot)_{\Omega^{\mathrm{s}}}$ (see, e.g., [30, Chapter 15]). We then set

$$
\begin{aligned}
& a_{h}^{\mathrm{e}}\left(\boldsymbol{d}_{h}, \boldsymbol{v}_{h}^{\mathrm{s}}\right) \stackrel{\text { def }}{=}\left(\boldsymbol{\sigma}\left(\boldsymbol{d}_{h}\right), \boldsymbol{\epsilon}\left(\boldsymbol{v}_{h}^{\mathrm{s}}\right)\right)_{\Omega^{\mathrm{s}}}+c_{0}\left(\boldsymbol{d}_{h}, \boldsymbol{v}_{h}^{\mathrm{s}}\right)_{\Omega^{\mathrm{s}}, h}, \\
& a_{h}^{\mathrm{v}}\left(\dot{\boldsymbol{d}}_{h}, \boldsymbol{v}_{h}^{\mathrm{s}}\right) \stackrel{\text { def }}{=} \beta\left(\boldsymbol{\sigma}\left(\dot{\boldsymbol{d}}_{h}\right), \boldsymbol{\epsilon}\left(\boldsymbol{v}_{h}^{\mathrm{s}}\right)\right)_{\Omega^{\mathrm{s}}}+\alpha \rho^{\mathrm{s}}\left(\dot{\boldsymbol{d}}_{h}, \boldsymbol{v}_{h}^{\mathrm{s}}\right)_{\Omega^{\mathrm{s}}, h}
\end{aligned}
$$

for all $\boldsymbol{d}_{h}, \dot{\boldsymbol{d}}_{h}, \boldsymbol{v}_{h}^{\mathrm{s}} \in \boldsymbol{V}_{h}^{\mathrm{s}}$.

The space semi-discrete formulation of problem (4), including a mass-lumping approximation in the structure, reads therefore as follows: for all $t>0$, find $\left(\boldsymbol{u}_{h}(t), \dot{\boldsymbol{d}}_{h}(t)\right) \in \boldsymbol{W}_{h}, p_{h}(t) \in Q_{h}$ and $\boldsymbol{d}_{h}(t) \in \boldsymbol{V}_{h}^{\text {s }}$ such that $\dot{\boldsymbol{d}}_{h}=\partial_{t} \boldsymbol{d}_{h}$ and

$$
\begin{aligned}
\rho^{\mathrm{f}}\left(\partial_{t} \boldsymbol{u}_{h}, \boldsymbol{v}_{h}^{\mathrm{f}}\right)_{\Omega^{\mathrm{f}}}+a\left(\boldsymbol{u}_{h}, \boldsymbol{v}_{h}^{\mathrm{f}}\right)+b\left(p_{h}, \boldsymbol{v}_{h}^{\mathrm{f}}\right)-b\left(q_{h}, \boldsymbol{u}_{h}\right)+s_{h}\left(p_{h}, q_{h}\right) \\
\quad+\rho^{\mathrm{s}}\left(\partial_{t} \dot{\boldsymbol{d}}_{h}, \boldsymbol{v}_{h}^{\mathrm{s}}\right)_{\Omega^{\mathrm{s}}, h}+a_{h}^{\mathrm{e}}\left(\boldsymbol{d}_{h}, \boldsymbol{v}_{h}^{\mathrm{s}}\right)+a_{h}^{\mathrm{v}}\left(\dot{\boldsymbol{d}}_{h}, \boldsymbol{v}_{h}^{\mathrm{s}}\right)=l\left(\boldsymbol{v}_{h}^{\mathrm{f}}\right)
\end{aligned}
$$

for all $\left(\boldsymbol{v}_{h}^{\mathrm{f}}, \boldsymbol{v}_{h}^{\mathrm{s}}\right) \in \boldsymbol{W}_{h}$ and $q_{h} \in Q_{h}$.

In what follows, we will consider the standard solid-sided and fluid-sided discrete lifting operators, $\mathcal{L}_{h}^{\mathrm{s}}: \boldsymbol{\Lambda}_{\Sigma, h} \rightarrow \boldsymbol{V}_{h}^{\mathrm{s}}$ and $\mathcal{L}_{h}^{\mathrm{f}}: \boldsymbol{\Lambda}_{\Sigma, h} \rightarrow \boldsymbol{V}_{h}^{\mathrm{f}}$, defined for all $\boldsymbol{\xi}_{h} \in \boldsymbol{\Lambda}_{\Sigma, h}$, such that the nodal values of $\mathcal{L}_{h}^{\mathrm{s}} \boldsymbol{\xi}_{h}, \mathcal{L}_{h}^{\mathrm{f}} \boldsymbol{\xi}_{h}$ vanish out of $\Sigma$ and that $\left.\left(\mathcal{L}_{h}^{\mathrm{s}} \boldsymbol{\xi}_{h}\right)\right|_{\Sigma}=\left.\left(\mathcal{L}_{h}^{\mathrm{f}} \boldsymbol{\xi}_{h}\right)\right|_{\Sigma}=\boldsymbol{\xi}_{h}$. We introduce also the interface operator $\boldsymbol{B}_{h}: \boldsymbol{\Lambda}_{\Sigma, h} \rightarrow \boldsymbol{\Lambda}_{\Sigma, h}$, defined by $\boldsymbol{B}_{h} \stackrel{\text { def }}{=}\left(\mathcal{L}_{h}^{\mathrm{s}}\right)^{\star} \mathcal{L}_{h}^{\mathrm{s}}$, where $\left(\mathcal{L}_{h}^{\mathrm{s}}\right)^{\star}$ stands for the adjoint operator of $\mathcal{L}_{h}^{\mathrm{s}}$ with respect to the lumped-mass inner product in $\boldsymbol{V}_{h}^{\mathrm{s}}$. Hence, we have

$$
\left(\boldsymbol{B}_{h} \boldsymbol{\xi}_{h}, \boldsymbol{\lambda}_{h}\right)_{\Sigma}=\left(\mathcal{L}_{h}^{\mathrm{s}} \boldsymbol{\xi}_{h}, \mathcal{L}_{h}^{\mathrm{s}} \boldsymbol{\lambda}_{h}\right)_{\Omega^{\mathrm{s}}, h}
$$

for all $\left(\boldsymbol{\xi}_{h}, \boldsymbol{\lambda}_{h}\right) \in \boldsymbol{\Lambda}_{\Sigma, h} \times \boldsymbol{\Lambda}_{\Sigma, h}$. An straightforward argument shows that the interface operator $\boldsymbol{B}_{h}$ is self-adjoint, positive definite and diagonal with respect to the finite element basis of $\boldsymbol{\Lambda}_{\Sigma, h}$.

Remark 1 In order to simplify the presentation, for $\boldsymbol{v}_{h}^{\mathrm{s}} \in \boldsymbol{V}_{h}^{\mathrm{s}}$, we will use the notation $\mathcal{L}_{h}^{\mathrm{s}} \boldsymbol{v}_{h}^{\mathrm{s}}$ instead of $\mathcal{L}_{h}^{\mathrm{s}}\left(\left.\boldsymbol{v}_{h}^{\mathrm{s}}\right|_{\Sigma}\right)$. The same applies to $\mathcal{L}_{h}^{\mathrm{f}}$ and $\boldsymbol{B}_{h}$. $\diamond$

In the next section, we will make extensive use of the following result.

Lemma 1 For all $\left(\boldsymbol{v}_{h}^{\mathrm{s}}, \boldsymbol{\xi}_{h}\right) \in \boldsymbol{V}_{h}^{\mathrm{s}} \times \boldsymbol{\Lambda}_{\Sigma, h}$, we have

$$
\left(\boldsymbol{v}_{h}^{\mathrm{s}}, \mathcal{L}_{h}^{\mathrm{s}} \boldsymbol{\xi}_{h}\right)_{\Omega^{\mathrm{s}}, h}=\left(\boldsymbol{B}_{h} \boldsymbol{v}_{h}^{\mathrm{s}}, \boldsymbol{\xi}_{h}\right)_{\Sigma} .
$$

Proof 1 For all $\boldsymbol{v}_{h}^{\mathrm{s}} \in \boldsymbol{V}_{h}^{\mathrm{s}}$, we consider the decomposition $\boldsymbol{v}_{h}^{\mathrm{s}}=\widetilde{\boldsymbol{v}}_{h}^{\mathrm{s}}+\mathcal{L}_{h}^{\mathrm{s}} \boldsymbol{v}_{h}^{\mathrm{s}}$, with $\widetilde{\boldsymbol{v}}_{h}^{\mathrm{s}} \in \boldsymbol{V}_{\Sigma, h}^{\mathrm{s}}$. We then observe that $\left(\widetilde{\boldsymbol{v}}_{h}^{\mathrm{s}}, \mathcal{L}_{h}^{\mathrm{s}} \boldsymbol{\xi}_{h}\right)_{\Omega^{\mathrm{s}}, h}=0$ for all $\boldsymbol{\xi}_{h} \in \boldsymbol{\Lambda}_{\Sigma, h}$, by the construction of the lumped-mass approximation. Hence, owing to (7), we have

$$
\left(\boldsymbol{v}_{h}^{\mathrm{s}}, \mathcal{L}_{h}^{\mathrm{s}} \boldsymbol{\xi}_{h}\right)_{\Omega^{\mathrm{s}}, h}=\left(\mathcal{L}_{h}^{\mathrm{s}} \boldsymbol{v}_{h}^{\mathrm{s}}, \mathcal{L}_{h}^{\mathrm{s}} \boldsymbol{\xi}_{h}\right)_{\Omega^{\mathrm{s}}, h}+\left(\widetilde{\boldsymbol{v}}_{h}^{\mathrm{s}}, \mathcal{L}_{h}^{\mathrm{s}} \boldsymbol{\xi}_{h}\right)_{\Omega^{\mathrm{s}}, h}=\left(\boldsymbol{B}_{h} \boldsymbol{v}_{h}^{\mathrm{s}}, \boldsymbol{\xi}_{h}\right)_{\Sigma}
$$

which completes the proof. $\diamond$ 


\subsection{Generalized interface Robin consistency}

The most basic partitioned procedures for the numerical solution of (1)-(3) are generally based on the following Dirichlet-Neumann formulation of problem (6): for $t>0$,

- Fluid (Dirichlet): find $\left(\boldsymbol{u}_{h}(t), p_{h}(t)\right) \in \boldsymbol{V}_{h}^{\mathrm{f}} \times Q_{h}$ such that

$$
\left\{\begin{array}{l}
\left.\boldsymbol{u}_{h}\right|_{\Sigma}=\left.\dot{\boldsymbol{d}}_{h}\right|_{\Sigma}, \\
\rho^{\mathrm{f}}\left(\partial_{t} \boldsymbol{u}_{h}, \widetilde{\boldsymbol{v}}_{h}^{\mathrm{f}}\right)_{\Omega^{\mathrm{f}}}+a\left(\boldsymbol{u}_{h}, \widetilde{\boldsymbol{v}}_{h}^{\mathrm{f}}\right)+b\left(p_{h}, \widetilde{\boldsymbol{v}}_{h}^{\mathrm{f}}\right)-b\left(q_{h}, \boldsymbol{u}_{h}\right)+s_{h}\left(p_{h}, q_{h}\right) \\
=l\left(\widetilde{\boldsymbol{v}}_{h}^{\mathrm{f}}\right)
\end{array}\right.
$$

for all $\left(\widetilde{\boldsymbol{v}}_{h}^{\mathrm{f}}, q_{h}\right) \in \boldsymbol{V}_{\Sigma, h}^{\mathrm{f}} \times Q_{h}$.

- Solid (Neumann): find $\left(\dot{\boldsymbol{d}}_{h}(t), \boldsymbol{d}_{h}(t)\right) \in \boldsymbol{V}_{h}^{\mathrm{s}} \times \boldsymbol{V}_{h}^{\mathrm{s}}$ such that

$$
\left\{\begin{array}{l}
\dot{\boldsymbol{d}}_{h}=\partial_{t} \boldsymbol{d}_{h}, \\
\rho^{\mathrm{s}}\left(\partial_{t} \dot{\boldsymbol{d}}_{h}, \boldsymbol{v}_{h}^{\mathrm{s}}\right)_{\Omega^{\mathrm{s}}, h}+a_{h}^{\mathrm{e}}\left(\boldsymbol{d}_{h}, \boldsymbol{v}_{h}^{\mathrm{s}}\right)+a_{h}^{\mathrm{v}}\left(\dot{\boldsymbol{d}}_{h}, \boldsymbol{v}_{h}^{\mathrm{s}}\right) \\
=-\rho^{\mathrm{f}}\left(\partial_{t} \boldsymbol{u}_{h}, \mathcal{L}_{h}^{\mathrm{f}} \boldsymbol{v}_{h}^{\mathrm{s}}\right)_{\Omega^{\mathrm{f}}}-a\left(\boldsymbol{u}_{h}, \mathcal{L}_{h}^{\mathrm{f}} \boldsymbol{v}_{h}^{\mathrm{s}}\right)-b\left(p_{h}, \mathcal{L}_{h}^{\mathrm{f}} \boldsymbol{v}_{h}^{\mathrm{s}}\right)
\end{array}\right.
$$

for all $\boldsymbol{v}_{h}^{\mathrm{s}} \in \boldsymbol{V}_{h}^{\mathrm{s}}$.

Unfortunately, explicit coupling schemes based on this fluid-solid splitting are known to yield severe added-mass stability issues (see, e.g., $[6,16]$ ). In the next paragraphs, we shall show that the monolithic problem (6) admits an alternative partitioned formulation based on (consistent) interface Robin conditions.

For this purpose, we test (10) with $\boldsymbol{v}_{h}^{\mathrm{s}}=\mathcal{L}_{h}^{\mathrm{s}} \boldsymbol{\xi}_{h}$ for all $\boldsymbol{\xi}_{h} \in \boldsymbol{\Lambda}_{\Sigma, h}$, to get

$$
\begin{aligned}
\rho^{\mathrm{f}}\left(\partial_{t} \boldsymbol{u}_{h}, \mathcal{L}_{h}^{\mathrm{f}} \boldsymbol{\xi}_{h}\right)_{\Omega^{\mathrm{f}}}+a\left(\boldsymbol{u}_{h}, \mathcal{L}_{h}^{\mathrm{f}} \boldsymbol{\xi}_{h}\right)+b\left(p_{h}, \mathcal{L}_{h}^{\mathrm{f}} \boldsymbol{\xi}_{h}\right) \\
\quad+\rho^{\mathrm{s}}\left(\partial_{t} \dot{\boldsymbol{d}}_{h}, \mathcal{L}_{h}^{\mathrm{s}} \boldsymbol{\xi}_{h}\right)_{\Omega^{\mathrm{s}}, h}+a_{h}^{\mathrm{e}}\left(\boldsymbol{d}_{h}, \mathcal{L}_{h}^{\mathrm{s}} \boldsymbol{\xi}_{h}\right)+a_{h}^{\mathrm{v}}\left(\dot{\boldsymbol{d}}_{h}, \mathcal{L}_{h}^{\mathrm{s}} \boldsymbol{\xi}_{h}\right)=0 .
\end{aligned}
$$

It should be noted that this relation is nothing but the spatial discrete counterpart of the interface kinetic condition $(3)_{2}$. Furthermore, since $\left.\boldsymbol{u}_{h}\right|_{\Sigma}=\left.\dot{\boldsymbol{d}}_{h}\right|_{\Sigma}$, from (8) we have

$$
\left(\partial_{t} \dot{\boldsymbol{d}}_{h}, \mathcal{L}_{h}^{\mathrm{s}} \boldsymbol{\xi}_{h}\right)_{\Omega^{\mathrm{s}}, h}=\left(\boldsymbol{B}_{h} \partial_{t} \dot{\boldsymbol{d}}_{h}, \boldsymbol{\xi}_{h}\right)_{\Sigma}=\left(\boldsymbol{B}_{h} \partial_{t} \boldsymbol{u}_{h}, \boldsymbol{\xi}_{h}\right)_{\Sigma}
$$

The relation (11) can thus be rewritten as

$$
\begin{aligned}
\rho^{\mathrm{f}}\left(\partial_{t} \boldsymbol{u}_{h}, \mathcal{L}_{h}^{\mathrm{f}} \boldsymbol{\xi}_{h}\right)_{\Omega^{\mathrm{f}}}+a\left(\boldsymbol{u}_{h}, \mathcal{L}_{h}^{\mathrm{f}} \boldsymbol{\xi}_{h}\right) & +b\left(p_{h}, \mathcal{L}_{h}^{\mathrm{f}} \boldsymbol{\xi}_{h}\right) \\
& +\rho^{\mathrm{s}}\left(\boldsymbol{B}_{h} \partial_{t} \boldsymbol{u}_{h}, \boldsymbol{\xi}_{h}\right)_{\Sigma}=-a_{h}^{\mathrm{e}}\left(\boldsymbol{d}_{h}, \mathcal{L}_{h}^{\mathrm{s}} \boldsymbol{\xi}_{h}\right)-a_{h}^{\mathrm{v}}\left(\dot{\boldsymbol{d}}_{h}, \mathcal{L}_{h}^{\mathrm{s}} \boldsymbol{\xi}_{h}\right)
\end{aligned}
$$

for all $\boldsymbol{\xi}_{h} \in \boldsymbol{\Lambda}_{\Sigma, h}$. Equivalently, the addition and subtraction of $\rho^{\mathrm{s}}\left(\partial_{t} \dot{\boldsymbol{d}}_{h}, \mathcal{L}_{h}^{\mathrm{s}} \boldsymbol{\xi}_{h}\right)_{\Omega^{\mathrm{s}}, h}$, in combination with (12), yields

$$
\begin{aligned}
\rho^{\mathrm{f}}\left(\partial_{t} \boldsymbol{u}_{h}, \mathcal{L}_{h}^{\mathrm{f}} \boldsymbol{\xi}_{h}\right)_{\Omega^{\mathrm{f}}}+a\left(\boldsymbol{u}_{h}, \mathcal{L}_{h}^{\mathrm{f}} \boldsymbol{\xi}_{h}\right) & +b\left(p_{h}, \mathcal{L}_{h}^{\mathrm{f}} \boldsymbol{\xi}_{h}\right) \\
+ & +\rho^{\mathrm{s}}\left(\boldsymbol{B}_{h} \partial_{t} \boldsymbol{u}_{h}, \boldsymbol{\xi}_{h}\right)_{\Sigma}=\rho^{\mathrm{s}}\left(\boldsymbol{B}_{h} \partial_{t} \dot{\boldsymbol{d}}_{h}, \boldsymbol{\xi}_{h}\right)_{\Sigma} \\
& -\left[\rho^{\mathrm{s}}\left(\partial_{t} \dot{\boldsymbol{d}}_{h}, \mathcal{L}_{h}^{\mathrm{s}} \boldsymbol{\xi}_{h}\right)_{\Omega^{\mathrm{s}}, h}+a_{h}^{\mathrm{e}}\left(\boldsymbol{d}_{h}, \mathcal{L}_{h}^{\mathrm{s}} \boldsymbol{\xi}_{h}\right)+a_{h}^{\mathrm{v}}\left(\dot{\boldsymbol{d}}_{h}, \mathcal{L}_{h}^{\mathrm{s}} \boldsymbol{\xi}_{h}\right)\right]
\end{aligned}
$$

for all $\boldsymbol{\xi}_{h} \in \boldsymbol{\Lambda}_{\Sigma, h}$.

$\mathrm{RR} \mathrm{n}^{\circ} 8384$ 
The preceding relation points out a major feature of the space semi-discrete solution given by (6): its intrinsic Robin consistency on the interface. Indeed, the identity (14) can formally be interpreted as the discrete counterpart of the generalized Robin condition

$$
\boldsymbol{\sigma}^{\mathrm{f}}(\boldsymbol{u}, p) \boldsymbol{n}^{\mathrm{f}}+\rho^{\mathrm{s}} \boldsymbol{B}_{h} \partial_{t} \boldsymbol{u}=\rho^{\mathrm{s}} \boldsymbol{B}_{h} \partial_{t} \dot{\boldsymbol{d}}-\boldsymbol{\sigma}^{\mathrm{s}}(\boldsymbol{d}, \dot{\boldsymbol{d}}) \boldsymbol{n}^{\mathrm{s}} \quad \text { on } \quad \Sigma .
$$

Note that, instead of the usual identity operator, the interface condition (15) involves the interface operator $\boldsymbol{B}_{h}$ defined by (7), hence the terminology generalized Robin.

By adding (14) to (9) we get the following Robin subproblem for the fluid: for $t>0$, find $\left(\boldsymbol{u}_{h}(t), p_{h}(t)\right) \in \boldsymbol{V}_{h}^{\mathrm{f}} \times Q_{h}$ such that

$$
\left\{\begin{array}{l}
\rho^{\mathrm{f}}\left(\partial_{t} \boldsymbol{u}_{h}, \boldsymbol{v}_{h}^{\mathrm{f}}\right)_{\Omega^{\mathrm{f}}}+a\left(\boldsymbol{u}_{h}, \boldsymbol{v}_{h}^{\mathrm{f}}\right)+b\left(p_{h}, \boldsymbol{v}_{h}^{\mathrm{f}}\right)-b\left(q_{h}, \boldsymbol{u}_{h}\right)+s_{h}\left(p_{h}, q_{h}\right) \\
\quad+\rho^{\mathrm{s}}\left(\boldsymbol{B}_{h} \partial_{t} \boldsymbol{u}_{h}, \boldsymbol{v}_{h}^{\mathrm{f}}\right)_{\Sigma}=\rho^{\mathrm{s}}\left(\boldsymbol{B}_{h} \partial_{t} \dot{\boldsymbol{d}}_{h}, \boldsymbol{v}_{h}^{\mathrm{f}}\right)_{\Sigma} \\
\quad-\left[\rho^{\mathrm{s}}\left(\partial_{t} \dot{\boldsymbol{d}}_{h}, \mathcal{L}_{h}^{\mathrm{s}} \boldsymbol{v}_{h}^{\mathrm{f}}\right)_{\Omega^{\mathrm{s}}, h}+a_{h}^{\mathrm{e}}\left(\boldsymbol{d}_{h}, \mathcal{L}_{h}^{\mathrm{s}} \boldsymbol{v}_{h}^{\mathrm{f}}\right)+a_{h}^{\mathrm{v}}\left(\dot{\boldsymbol{d}}_{h}, \mathcal{L}_{h}^{\mathrm{s}} \boldsymbol{v}_{h}^{\mathrm{f}}\right)\right]+l\left(\boldsymbol{v}_{h}^{\mathrm{f}}\right)
\end{array}\right.
$$

for all $\left(\boldsymbol{v}_{h}^{\mathrm{f}}, q_{h}\right) \in \boldsymbol{V}_{h}^{\mathrm{f}} \times Q_{h}$.

Therefore, instead of formulating the fluid-solid time-splitting from the traditional DirichletNeumann coupling (9)-(10), in this work we consider the Robin-Neumann formulation given by (16) and (10). As in [13], we will see that the benefits of this approach are threefold:

- the implicit treatment of the interface solid inertial term in the left-hand side of (15) is enough to guarantee (added-mass free) stability;

- the explicit treatment of the right-hand side of (15) enables the full fluid-solid splitting without compromising stability;

- the resulting schemes are genuine partitioned methods with an intrinsic (i.e., parameter free) explicit Robin-Neumann pattern.

\subsection{Time discretization: explicit coupling schemes}

In what follows, the parameter $\tau>0$ stands for the time-step length, $t_{n} \stackrel{\text { def }}{=} n \tau$, for $n \in \mathbb{N}$, and $\partial_{\tau} x^{n} \stackrel{\text { def }}{=}\left(x^{n}-x^{n-1}\right) / \tau$ for the first-order backward difference. The symbol $x^{\star}$ denotes the $r$-order extrapolation of $x^{n}$, namely,

$$
x^{\star} \stackrel{\text { def }}{=}\left\{\begin{array}{lll}
0 & \text { if } & r=0, \\
x^{n-1} & \text { if } & r=1, \\
2 x^{n-1}-x^{n-2} & \text { if } & r=2 .
\end{array}\right.
$$

The fully discrete approximation of (1)-(3) is split into the following sequential sub-steps: for $n \geq r+1$,

1. Fluid step (generalized Robin): find $\left(\boldsymbol{u}_{h}^{n}, p_{h}^{n}\right) \in \boldsymbol{V}_{h}^{\mathrm{f}} \times Q_{h}$ such that

$$
\left\{\begin{array}{l}
\rho^{\mathrm{f}}\left(\partial_{\tau} \boldsymbol{u}_{h}^{n}, \boldsymbol{v}_{h}^{\mathrm{f}}\right)_{\Omega^{\mathrm{f}}}+a\left(\boldsymbol{u}_{h}^{n}, \boldsymbol{v}_{h}^{\mathrm{f}}\right)+b\left(p_{h}^{n}, \boldsymbol{v}_{h}^{\mathrm{f}}\right)-b\left(q_{h}, \boldsymbol{u}_{h}^{n}\right)+s_{h}\left(p_{h}^{n}, q_{h}\right) \\
+\frac{\rho^{\mathrm{s}}}{\tau}\left(\boldsymbol{B}_{h} \boldsymbol{u}_{h}^{n}, \boldsymbol{v}_{h}^{\mathrm{f}}\right)_{\Sigma}=\frac{\rho^{\mathrm{s}}}{\tau}\left(\boldsymbol{B}_{h}\left(\dot{\boldsymbol{d}}_{h}^{n-1}+\tau \partial_{\tau} \dot{\boldsymbol{d}}_{h}^{\star}\right), \boldsymbol{v}_{h}^{\mathrm{f}}\right)_{\Sigma} \\
\quad-\left[\rho^{\mathrm{s}}\left(\partial_{\tau} \dot{\boldsymbol{d}}_{h}^{\star}, \mathcal{L}_{h}^{\mathrm{s}} \boldsymbol{v}_{h}^{\mathrm{f}}\right)_{\Omega^{\mathrm{s}}, h}+a_{h}^{\mathrm{e}}\left(\boldsymbol{d}_{h}^{\star}, \mathcal{L}_{h}^{\mathrm{s}} \boldsymbol{v}_{h}^{\mathrm{f}}\right)+a_{h}^{\mathrm{v}}\left(\dot{\boldsymbol{d}}_{h}^{\star}, \mathcal{L}_{h}^{\mathrm{s}} \boldsymbol{v}_{h}^{\mathrm{f}}\right)\right]+l\left(\boldsymbol{v}_{h}^{\mathrm{f}}\right)
\end{array}\right.
$$

for all $\left(\boldsymbol{v}_{h}^{\mathrm{f}}, q_{h}\right) \in \boldsymbol{V}_{h}^{\mathrm{f}} \times Q_{h}$. 
2. Solid step (Neumann): find $\left(\dot{\boldsymbol{d}}_{h}^{n}, \boldsymbol{d}_{h}^{n}\right) \in \boldsymbol{V}_{h}^{\mathrm{s}} \times \boldsymbol{V}_{h}^{\mathrm{s}}$ such that

$$
\left\{\begin{array}{l}
\dot{\boldsymbol{d}}_{h}^{n}=\partial_{\tau} \boldsymbol{d}_{h}^{n}, \\
\rho^{\mathrm{s}}\left(\partial_{\tau} \dot{\boldsymbol{d}}_{h}^{n}, \boldsymbol{v}_{h}^{\mathrm{s}}\right)_{\Omega^{\mathrm{s}}, h}+a_{h}^{\mathrm{e}}\left(\boldsymbol{d}_{h}^{n}, \boldsymbol{v}_{h}^{\mathrm{s}}\right)+a_{h}^{\mathrm{v}}\left(\dot{\boldsymbol{d}}_{h}^{n}, \boldsymbol{v}_{h}^{\mathrm{s}}\right) \\
=-\rho^{\mathrm{f}}\left(\partial_{\tau} \boldsymbol{u}_{h}^{n}, \mathcal{L}_{h}^{\mathrm{f}} \boldsymbol{v}_{h}^{\mathrm{s}}\right)_{\Omega^{\mathrm{f}}}-a\left(\boldsymbol{u}_{h}^{n}, \mathcal{L}_{h}^{\mathrm{f}} \boldsymbol{v}_{h}^{\mathrm{s}}\right)-b\left(p_{h}^{n}, \mathcal{L}_{h}^{\mathrm{f}} \boldsymbol{v}_{h}^{\mathrm{s}}\right)
\end{array}\right.
$$

for all $\boldsymbol{v}_{h}^{\mathrm{s}} \in \boldsymbol{V}_{h}^{\mathrm{s}}$.

In strong form, these two steps perform respectively the following time-marching on the interface

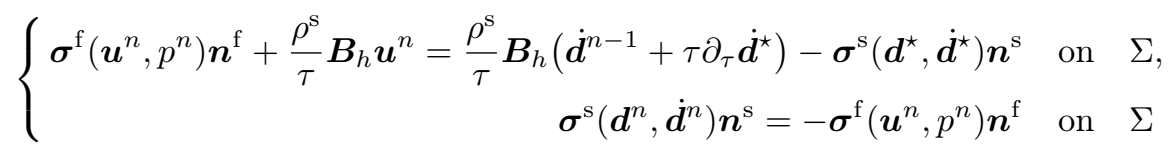

for $n \geq r+1$. The fundamental ingredient of the splitting (17)-(18) is the generalized explicit Robin condition $(19)_{1}$, which has been derived as a specific semi-implicit time discretization of (15):

- the solid contributions are treated explicitly via extrapolation in the right-hand side of (15). This provides the uncoupling of the fluid and solid time-marchings (17)-(18);

- the interface solid inertia is treated implicitly in the left-hand side of (15). This guarantees (added-mass free) stability.

Remark 2 The time-splitting induced by (19) is consistent with the original interface coupling (3), in the sense that it can be interpreted as a time discretization of the equivalent interface relations

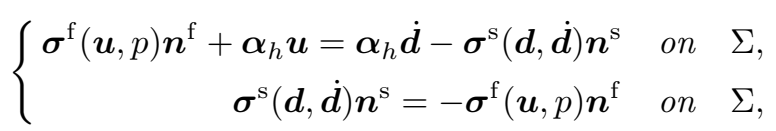

with the invertible interface operator $\boldsymbol{\alpha}_{h} \stackrel{\text { def }}{=} \rho^{\mathrm{s}} \tau^{-1} \boldsymbol{B}_{h}$. The right-hand side of $(19)_{1}$ is simply an explicit approximation of the right-hand side of $(20)_{1}$. Moreover, owing to $(19)_{2}$, the role of the generalized Robin condition $(19)_{1}$ is the enforcement of the kinematic continuity $(3)_{1}$.

\begin{tabular}{|c|c|c|}
\hline$r$ & $\dot{\boldsymbol{d}}^{n-1}+\tau \partial_{\tau} \dot{\boldsymbol{d}}^{\star}$ & $\boldsymbol{\sigma}^{\mathrm{s}}\left(\boldsymbol{d}^{\star}, \dot{\boldsymbol{d}}^{\star}\right) \boldsymbol{n}^{\mathrm{s}}$ \\
\hline 0 & $\dot{\boldsymbol{d}}^{n-1}$ & $\mathbf{0}$ \\
1 & $2 \dot{\boldsymbol{d}}^{n-1}-\dot{\boldsymbol{d}}^{n-2}$ & $\boldsymbol{\sigma}^{\mathrm{s}}\left(\boldsymbol{d}^{n-1}, \dot{\boldsymbol{d}}^{n-1}\right) \boldsymbol{n}^{\mathrm{s}}$ \\
2 & $3 \dot{\boldsymbol{d}}^{n-1}-3 \dot{\boldsymbol{d}}^{n-2}+\dot{\boldsymbol{d}}^{n-3}$ & $2 \boldsymbol{\sigma}^{\mathrm{s}}\left(\boldsymbol{d}^{n-1}, \dot{\boldsymbol{d}}^{n-1}\right) \boldsymbol{n}^{\mathrm{s}}-\boldsymbol{\sigma}^{\mathrm{s}}\left(\boldsymbol{d}^{n-2}, \dot{\boldsymbol{d}}^{n-2}\right) \boldsymbol{n}^{\mathrm{s}}$ \\
\hline
\end{tabular}

Table 1: Extrapolations of the interface solid velocity and stress considered in (21).

For the sake of clarity, the strong form of the explicit coupling schemes (17)-(18) is presented in Algorithm 1. The corresponding extrapolations within the fluid step are listed in Table 1. Note that, for $r=1$ and 2, the schemes are multi-steps methods. The additional data needed to start the time-marching can be generated by performing one step of the scheme with $r=0$, which yields $\left(\boldsymbol{d}^{1}, \dot{\boldsymbol{d}}^{1}\right)$, and then one step of the scheme with $r=1$, which gives $\left(\boldsymbol{d}^{2}, \dot{\boldsymbol{d}}^{2}\right)$.

Remark 3 Owing to the above initialization procedure and to $(22)_{4}$, we have

$$
\boldsymbol{\sigma}^{\mathrm{f}}\left(\boldsymbol{u}^{\star}, p^{\star}\right) \boldsymbol{n}^{\mathrm{f}}=-\boldsymbol{\sigma}^{\mathrm{s}}\left(\boldsymbol{d}^{\star}, \dot{\boldsymbol{d}}^{\star}\right) \boldsymbol{n}^{\mathrm{s}} \quad \text { on } \quad \Sigma
$$


Algorithm 1 Generalized Robin-Neumann explicit coupling schemes.

For $n \geq r+1$,

1. Fluid step (generalized Robin): find $\boldsymbol{u}^{n}: \Omega^{\mathrm{f}} \times \mathbb{R}^{+} \rightarrow \mathbb{R}^{d}$ and $p^{n}: \Omega^{\mathrm{f}} \times \mathbb{R}^{+} \rightarrow \mathbb{R}$ such that

$$
\left\{\begin{array}{rcc}
\rho^{\mathrm{f}} \partial_{\tau} \boldsymbol{u}^{n}-\operatorname{div} \boldsymbol{\sigma}^{\mathrm{f}}\left(\boldsymbol{u}^{n}, p^{n}\right)=\mathbf{0} & \text { in } \quad \Omega^{\mathrm{f}}, \\
\operatorname{div} \boldsymbol{u}^{n}=0 & \text { in } \quad \Omega^{\mathrm{f}}, \\
\boldsymbol{\sigma}^{\mathrm{f}}\left(\boldsymbol{u}^{n}, p^{n}\right) \boldsymbol{n}^{\mathrm{f}}=\boldsymbol{f}^{\mathrm{\Gamma}}\left(t_{n}\right) & \text { on } \quad \Gamma, \\
\boldsymbol{\sigma}^{\mathrm{f}}\left(\boldsymbol{u}^{n}, p^{n}\right) \boldsymbol{n}^{\mathrm{f}}+\frac{\rho^{\mathrm{s}}}{\tau} \boldsymbol{B}_{h} \boldsymbol{u}^{n}=\frac{\rho^{\mathrm{s}}}{\tau} \boldsymbol{B}_{h}\left(\dot{\boldsymbol{d}}^{n-1}+\tau \partial_{\tau} \dot{\boldsymbol{d}}^{\star}\right) & & \\
-\boldsymbol{\sigma}^{\mathrm{s}}\left(\boldsymbol{d}^{\star}, \dot{\boldsymbol{d}}^{\star}\right) \boldsymbol{n}^{\mathrm{s}} & \text { on } \quad \Sigma .
\end{array}\right.
$$

2. Solid step (Neumann): find $\boldsymbol{d}^{n}: \Omega^{\mathrm{s}} \times \mathbb{R}^{+} \rightarrow \mathbb{R}^{d}$ and $\dot{\boldsymbol{d}}^{n}: \Omega^{\mathrm{s}} \times \mathbb{R}^{+} \rightarrow \mathbb{R}^{d}$ such that

$$
\left\{\begin{array}{rccc}
\rho^{\mathrm{s}} \partial_{\tau} \dot{\boldsymbol{d}}^{n}+\alpha \rho^{\mathrm{s}} \dot{\boldsymbol{d}}^{n}-\operatorname{div} \boldsymbol{\sigma}^{\mathrm{s}}\left(\boldsymbol{d}^{n}, \dot{\boldsymbol{d}}^{n}\right)+c_{0} \boldsymbol{d}^{n}=\mathbf{0} & \text { in } & \Omega^{\mathrm{s}}, \\
\boldsymbol{d}^{n}=\mathbf{0}, \beta \dot{\boldsymbol{d}}^{n}=\mathbf{0} & \text { on } & \Gamma^{\mathrm{d}}, \\
\boldsymbol{\sigma}^{\mathrm{s}}\left(\boldsymbol{d}^{n}, \dot{\boldsymbol{d}}^{n}\right) \boldsymbol{n}^{\mathrm{s}}=\mathbf{0} & \text { on } & \Gamma^{\mathrm{n}}, \\
\boldsymbol{\sigma}^{\mathrm{s}}\left(\boldsymbol{d}^{n}, \dot{\boldsymbol{d}}^{n}\right) \boldsymbol{n}^{\mathrm{s}}=-\boldsymbol{\sigma}^{\mathrm{f}}\left(\boldsymbol{u}^{n}, p^{n}\right) \boldsymbol{n}^{\mathrm{f}} & \text { on } & \Sigma .
\end{array}\right.
$$

for $n \geq r+1$. Therefore, the generalized interface Robin condition $(21)_{4}$ can be rewritten as

$$
\boldsymbol{\sigma}^{\mathrm{f}}\left(\boldsymbol{u}^{n}, p^{n}\right) \boldsymbol{n}^{\mathrm{f}}+\frac{\rho^{\mathrm{s}}}{\tau} \boldsymbol{B}_{h} \boldsymbol{u}^{n}=\frac{\rho^{\mathrm{s}}}{\tau} \boldsymbol{B}_{h}\left(\dot{\boldsymbol{d}}^{n-1}+\tau \partial_{\tau} \dot{\boldsymbol{d}}^{\star}\right)+\boldsymbol{\sigma}^{\mathrm{f}}\left(\boldsymbol{u}^{\star}, p^{\star}\right) \boldsymbol{n}^{\mathrm{f}} \quad \text { on } \quad \Sigma
$$

for $n \geq r+1$. The advantage with this equivalent formulation is that only interface solid velocities have to be transferred to the fluid in Algorithm 1, as in standard partitioned Dirichlet-Neumann procedures. $\diamond$

\subsection{Partitioned solution of implicit coupling}

The explicit coupling schemes (17)-(18) can be viewed as a single iteration (with appropriate initializations) of a new Robin-Neumann iterative method for the partitioned solution of the following implicit coupling scheme: for $n \geq 1$, find $\left(\boldsymbol{u}_{h}^{n}, \dot{\boldsymbol{d}}_{h}^{n}\right) \in \boldsymbol{W}_{h}, p_{h}^{n} \in Q_{h}$ and $\boldsymbol{d}_{h}^{n} \in \boldsymbol{V}_{h}^{\mathrm{s}}$ such that $\dot{\boldsymbol{d}}_{h}^{n}=\partial_{\tau} \boldsymbol{d}_{h}^{n}$ and

$$
\begin{array}{r}
\rho^{\mathrm{f}}\left(\partial_{\tau} \boldsymbol{u}_{h}^{n}, \boldsymbol{v}_{h}^{\mathrm{f}}\right)_{\Omega^{\mathrm{f}}}+a\left(\boldsymbol{u}_{h}^{n}, \boldsymbol{v}_{h}^{\mathrm{f}}\right)+b\left(p_{h}^{n}, \boldsymbol{v}_{h}^{\mathrm{f}}\right)-b\left(q_{h}, \boldsymbol{u}_{h}^{n}\right)+s_{h}\left(p_{h}^{n}, q_{h}\right) \\
+\rho^{\mathrm{s}}\left(\partial_{\tau} \dot{\boldsymbol{d}}_{h}^{n}, \boldsymbol{v}_{h}^{\mathrm{s}}\right)_{\Omega^{\mathrm{s}}, h}+a_{h}^{\mathrm{e}}\left(\boldsymbol{d}_{h}^{n}, \boldsymbol{v}_{h}^{\mathrm{s}}\right)+a_{h}^{\mathrm{v}}\left(\dot{\boldsymbol{d}}_{h}^{n}, \boldsymbol{v}_{h}^{\mathrm{s}}\right)=l\left(\boldsymbol{v}_{h}^{\mathrm{f}}\right)
\end{array}
$$

for all $\left(\boldsymbol{v}_{h}^{\mathrm{f}}, \boldsymbol{v}_{h}^{\mathrm{s}}\right) \in \boldsymbol{W}_{h}$ and $q_{h} \in Q_{h}$. The corresponding generalized Robin-Neumann iterations read as follows:

1. Initialize $\dot{\boldsymbol{d}}_{h, 0}$ and $\boldsymbol{d}_{h, 0}$.

2. For $k=1, \ldots$ until convergence: 
- Fluid (generalized Robin): find $\left(\boldsymbol{u}_{h, k}, p_{h, k}\right) \in \boldsymbol{V}_{h}^{\mathrm{f}} \times Q_{h}$ such that

$$
\left\{\begin{array}{l}
\frac{\rho^{\mathrm{f}}}{\tau}\left(\boldsymbol{u}_{h, k}-\boldsymbol{u}_{h}^{n-1}, \boldsymbol{v}_{h}^{\mathrm{f}}\right)_{\Omega^{\mathrm{f}}}+a\left(\boldsymbol{u}_{h, k}, \boldsymbol{v}_{h}^{\mathrm{f}}\right)+b\left(p_{h, k}, \boldsymbol{v}_{h}^{\mathrm{f}}\right)-b\left(q_{h}, \boldsymbol{u}_{h, k}\right) \\
+s_{h}\left(p_{h, k}, q_{h}\right)+\frac{\rho^{\mathrm{s}}}{\tau}\left(\boldsymbol{B}_{h} \boldsymbol{u}_{h, k}, \boldsymbol{v}_{h}^{\mathrm{f}}\right)_{\Sigma}=\frac{\rho^{\mathrm{s}}}{\tau}\left(\boldsymbol{B}_{h} \dot{\boldsymbol{d}}_{h, k-1}, \boldsymbol{v}_{h}^{\mathrm{f}}\right)_{\Sigma} \\
\quad-\frac{\rho^{\mathrm{s}}}{\tau}\left(\dot{\boldsymbol{d}}_{h, k-1}-\dot{\boldsymbol{d}}_{h}^{n-1}, \mathcal{L}_{h}^{\mathrm{s}} \boldsymbol{v}_{h}^{\mathrm{f}}\right)_{\Omega^{\mathrm{s}}, h}-a_{h}^{\mathrm{e}}\left(\boldsymbol{d}_{h, k-1}, \mathcal{L}_{h}^{\mathrm{s}} \boldsymbol{v}_{h}^{\mathrm{f}}\right) \\
\quad-a_{h}^{\mathrm{v}}\left(\dot{\boldsymbol{d}}_{h, k-1}, \mathcal{L}_{h}^{\mathrm{s}} \boldsymbol{v}_{h}^{\mathrm{f}}\right)+l\left(\boldsymbol{v}_{h}^{\mathrm{f}}\right)
\end{array}\right.
$$

for all $\left(\boldsymbol{v}_{h}^{\mathrm{f}}, q_{h}\right) \in \boldsymbol{V}_{h}^{\mathrm{f}} \times Q_{h}$.

- Solid (Neumann): find $\left(\dot{\boldsymbol{d}}_{h, k}, \boldsymbol{d}_{h, k}\right) \in \boldsymbol{V}_{h}^{\mathrm{s}} \times \boldsymbol{V}_{h}^{\mathrm{s}}$ such that

$$
\left\{\begin{array}{l}
\dot{\boldsymbol{d}}_{h, k}=\frac{\boldsymbol{d}_{h, k}-\boldsymbol{d}_{h}^{n-1}}{\tau}, \\
\frac{\rho^{\mathrm{s}}}{\tau}\left(\dot{\boldsymbol{d}}_{h, k}-\dot{\boldsymbol{d}}_{h}^{n-1}, \boldsymbol{v}_{h}^{\mathrm{s}}\right)_{\Omega^{\mathrm{s}}, h}+a_{h}^{\mathrm{e}}\left(\boldsymbol{d}_{h, k}, \boldsymbol{v}_{h}^{\mathrm{s}}\right)+a_{h}^{\mathrm{v}}\left(\dot{\boldsymbol{d}}_{h, k}, \boldsymbol{v}_{h}^{\mathrm{s}}\right) \\
=-\frac{\rho^{\mathrm{f}}}{\tau}\left(\boldsymbol{u}_{h, k}-\boldsymbol{u}_{h}^{n-1}, \mathcal{L}_{h}^{\mathrm{f}} \boldsymbol{v}_{h}^{\mathrm{s}}\right)_{\Omega^{\mathrm{f}}}-a\left(\boldsymbol{u}_{h, k}, \mathcal{L}_{h}^{\mathrm{f}} \boldsymbol{v}_{h}^{\mathrm{s}}\right) \\
\quad-b\left(p_{h, k}, \mathcal{L}_{h}^{\mathrm{f}} \boldsymbol{v}_{h}^{\mathrm{s}}\right)
\end{array}\right.
$$

for all $\boldsymbol{v}_{h}^{\mathrm{s}} \in \boldsymbol{V}_{h}^{\mathrm{s}}$.

\section{Algorithm 2 Partitioned Robin-Neumann iterations based on Algorithm 1}

1. Initialize $\left.\boldsymbol{d}_{0}\right|_{\Sigma}$ and $\left.\boldsymbol{\sigma}^{\mathrm{s}}\left(\boldsymbol{d}_{0}, \dot{\boldsymbol{d}}_{0}\right) \boldsymbol{n}^{\mathrm{s}}\right|_{\Sigma}$.

2. For $k=1, \ldots$ until convergence:

- Fluid: find $\boldsymbol{u}_{k}: \Omega^{\mathrm{f}} \times \mathbb{R}^{+} \rightarrow \mathbb{R}^{d}$ and $p_{k}: \Omega^{\mathrm{f}} \times \mathbb{R}^{+} \rightarrow \mathbb{R}$ such that

$$
\left\{\begin{array}{rc}
\frac{\rho^{\mathrm{f}}}{\tau}\left(\boldsymbol{u}_{k}-\boldsymbol{u}^{n-1}\right)-\operatorname{div} \boldsymbol{\sigma}^{\mathrm{f}}\left(\boldsymbol{u}_{k}, p_{k}\right)=\mathbf{0} & \text { in } \quad \Omega^{\mathrm{f}} \\
\operatorname{div} \boldsymbol{u}_{k}=0 & \text { in } \quad \Omega^{\mathrm{f}}, \\
\boldsymbol{\sigma}^{\mathrm{f}}\left(\boldsymbol{u}_{k}, p_{k}\right) \boldsymbol{n}^{\mathrm{f}}=\boldsymbol{f}^{\Gamma}\left(t_{n}\right) & \text { on } \quad \Gamma, \\
\boldsymbol{\sigma}^{\mathrm{f}}\left(\boldsymbol{u}_{k}, p_{k}\right) \boldsymbol{n}_{\mathrm{f}}+\frac{\rho^{\mathrm{s}}}{\tau} \boldsymbol{B}_{h} \boldsymbol{u}_{k}=\frac{\rho^{\mathrm{s}}}{\tau} \boldsymbol{B}_{h} \dot{\boldsymbol{d}}_{k-1} & \\
-\boldsymbol{\sigma}^{\mathrm{s}}\left(\boldsymbol{d}_{k-1}, \dot{\boldsymbol{d}}_{k-1}\right) \boldsymbol{n}^{\mathrm{s}} & \text { on } \Sigma .
\end{array}\right.
$$

- Solid: find $\boldsymbol{d}_{k}: \Omega^{\mathrm{s}} \times \mathbb{R}^{+} \rightarrow \mathbb{R}^{d}$ and $\dot{\boldsymbol{d}}_{k}: \Omega^{\mathrm{s}} \times \mathbb{R}^{+} \rightarrow \mathbb{R}^{d}$ such that $\dot{\boldsymbol{d}}_{k}=\left(\boldsymbol{d}_{k}-\boldsymbol{d}^{n-1}\right) / \tau$ and

$$
\left\{\begin{array}{rccc}
\frac{\rho^{\mathrm{s}}}{\tau}\left(\dot{\boldsymbol{d}}_{k}-\dot{\boldsymbol{d}}^{n-1}\right)+\alpha \rho^{\mathrm{s}} \dot{\boldsymbol{d}}_{k}-\operatorname{div} \boldsymbol{\sigma}^{\mathrm{s}}\left(\boldsymbol{d}_{k}, \dot{\boldsymbol{d}}_{k}\right)+c_{0} \boldsymbol{d}_{k}=\mathbf{0} & \text { in } & \Omega^{\mathrm{s}} \\
\boldsymbol{d}_{k}=\mathbf{0}, \beta \dot{\boldsymbol{d}}_{k}=\mathbf{0} & \text { on } & \Gamma^{\mathrm{d}}, \\
\boldsymbol{\sigma}^{\mathrm{s}}\left(\boldsymbol{d}_{k}, \dot{\boldsymbol{d}}_{k}\right) \boldsymbol{n}^{\mathrm{s}}=\mathbf{0} & \text { on } & \Gamma^{\mathrm{n}} \\
\boldsymbol{\sigma}^{\mathrm{s}}\left(\boldsymbol{d}_{k}, \dot{\boldsymbol{d}}_{k}\right) \boldsymbol{n}^{\mathrm{s}}=-\boldsymbol{\sigma}^{\mathrm{f}}\left(\boldsymbol{u}_{k}, p_{k}\right) \boldsymbol{n}^{\mathrm{f}} & \text { on } & \Sigma .
\end{array}\right.
$$


For the sake of clarity, the strong form of the above iterative procedure is reported in Algorithm 2 .

Remark 4 Unlike traditional Robin based procedures (see, e.g., [1]), Algorithm 2 is parameter free. This is of fundamental importance in practice, since inappropriate choices of free Robin parameters are known to yield slow convergence or even divergent behavior. Another key difference has to do with the interface operator $\boldsymbol{B}_{h}$, which here is not proportional to the identity (as usual). In fact, the underlying structure of $\boldsymbol{B}_{h}$ comes from the generalized Robin consistency (15) at the space semi-discrete level. In Section 4.3, we will see that this guarantees the convergence of the iterations.

\section{Numerical analysis}

This section is devoted to the numerical analysis of the generalized Robin-Neumann methods introduced above. The stability of the explicit coupling schemes (17)-(18) is the topic of Section 4.2. In Section 4.3 we address the convergence of the iterative procedure (24)-(25).

\subsection{Preliminaries}

In what follows, the symbols $\lesssim$ and $\gtrsim$ indicate inequalities up to a multiplicative constant (independent of the physical and discretization parameters). We denote by $\|\cdot\|_{\mathrm{e}},\|\cdot\|_{\mathrm{v}},\|\cdot\|_{\mathrm{e}, h}$, $\|\cdot\|_{\mathrm{v}, h}$ and $\|\cdot\|_{\mathrm{s}, h}$ the norms associated to the inner-products $a^{\mathrm{e}}, a^{\mathrm{v}}, a_{h}^{\mathrm{e}}, a_{h}^{\mathrm{v}}$ and $(\cdot, \cdot)_{\Omega^{\mathrm{s}}, h}$, respectively.

Remark 5 The norms $\|\cdot\|_{0, \Omega^{\mathrm{s}}}$ and $\|\cdot\|_{\mathrm{s}, h}$ are equivalent in $\boldsymbol{V}_{h}^{\mathrm{s}}$, uniformly in $h$ (see, e.g., [30, Chapter 15]). As a result, the same holds for $\|\cdot\|_{\mathrm{e}}\left(\right.$ resp. $\left.\|\cdot\|_{\mathrm{v}}\right)$ and $\|\cdot\|_{\mathrm{e}, h}\left(\right.$ resp. $\left.\|\cdot\|_{\mathrm{v}, h}\right)$.

We consider discrete reconstructions, $\boldsymbol{L}_{h}^{\mathrm{e}}: \boldsymbol{V}^{\mathrm{s}} \rightarrow \boldsymbol{V}_{h}^{\mathrm{s}}$ and $\boldsymbol{L}_{h}^{\mathrm{v}}: \boldsymbol{V}^{\mathrm{s}} \rightarrow \boldsymbol{V}_{h}^{\mathrm{s}}$, of the elastic and viscous solid operators, defined through the relations:

$$
\left(\boldsymbol{L}_{h}^{\mathrm{e}} \boldsymbol{d}, \boldsymbol{v}_{h}^{\mathrm{s}}\right)_{\Omega^{\mathrm{s}}, h}=a_{h}^{\mathrm{e}}\left(\boldsymbol{d}, \boldsymbol{v}_{h}^{\mathrm{s}}\right), \quad\left(\boldsymbol{L}_{h}^{\mathrm{v}} \dot{\boldsymbol{d}}, \boldsymbol{v}_{h}^{\mathrm{s}}\right)_{\Omega^{\mathrm{s}}, h}=a_{h}^{\mathrm{v}}\left(\dot{\boldsymbol{d}}, \boldsymbol{v}_{h}^{\mathrm{s}}\right)
$$

for all $\left(\boldsymbol{d}, \dot{\boldsymbol{d}}, \boldsymbol{v}_{h}^{\mathrm{s}}\right) \in \boldsymbol{V}^{\mathrm{s}} \times \boldsymbol{V}^{\mathrm{s}} \times \boldsymbol{V}_{h}^{\mathrm{s}}$. Owing to Remark 5 , there exists a positive constant $\beta^{\mathrm{e}}$ such that

$$
a_{h}^{\mathrm{e}}\left(\boldsymbol{d}_{h}, \boldsymbol{d}_{h}\right) \leq \beta^{\mathrm{e}}\left\|\boldsymbol{d}_{h}\right\|_{1, \Omega^{\mathrm{s}}}^{2}
$$

for all $\boldsymbol{d}_{h} \in \boldsymbol{V}_{h}^{\mathrm{s}}$. Furthermore, using an inverse inequality between the norms $\|\cdot\|_{1, \Omega^{\mathrm{s}}}$ and $\|\cdot\|_{\mathrm{s}, h}$, whose constant is denoted by $C_{\text {inv }}$, we have the following estimates

$$
\begin{aligned}
&\left\|\boldsymbol{v}_{h}^{\mathrm{s}}\right\|_{\mathrm{e}, h}^{2} \leq \frac{\beta_{\mathrm{e}} C_{\mathrm{inv}}^{2}}{h^{2}}\left\|\boldsymbol{v}_{h}^{\mathrm{s}}\right\|_{\mathrm{s}, h}^{2}, \quad\left\|\boldsymbol{L}_{h}^{\mathrm{e}} \boldsymbol{v}_{h}^{\mathrm{s}}\right\|_{\mathrm{e}, h} \leq \frac{\beta_{\mathrm{e}} C_{\mathrm{inv}}^{2}}{h^{2}}\left\|\boldsymbol{v}_{h}^{\mathrm{s}}\right\|_{\mathrm{e}, h}, \\
&\left\|\boldsymbol{L}_{h}^{\mathrm{e}} \boldsymbol{v}_{h}^{\mathrm{s}}\right\|_{\mathrm{s}, h}^{2} \leq \frac{\beta_{\mathrm{e}} C_{\mathrm{inv}}^{2}}{h^{2}}\left\|\boldsymbol{v}_{h}^{\mathrm{s}}\right\|_{\mathrm{e}, h}^{2}, \quad\left\|\boldsymbol{v}_{h}^{\mathrm{s}}\right\|_{\mathrm{v}, h}^{2} \leq\left(\alpha \rho^{\mathrm{s}}+\beta \frac{\beta_{\mathrm{e}} C_{\mathrm{inv}}^{2}}{h^{2}}\right)\left\|\boldsymbol{v}_{h}^{\mathrm{s}}\right\|_{\mathrm{s}, h}^{2}, \\
&\left\|\boldsymbol{L}_{h}^{\mathrm{v}} \boldsymbol{v}_{h}^{\mathrm{s}}\right\|_{\mathrm{v}, h} \leq\left(\alpha \rho^{\mathrm{s}}+\beta \frac{\beta_{\mathrm{e}} C_{\mathrm{inv}}^{2}}{h^{2}}\right)\left\|\boldsymbol{v}_{h}^{\mathrm{s}}\right\|_{\mathrm{v}, h} \\
&\left\|\boldsymbol{L}_{h}^{\mathrm{v}} \boldsymbol{v}_{h}^{\mathrm{s}}\right\|_{\mathrm{s}, h}^{2} \leq\left(\alpha \rho^{\mathrm{s}}+\beta \frac{\beta_{\mathrm{e}} C_{\mathrm{inv}}^{2}}{h^{2}}\right)\left\|\boldsymbol{v}_{h}^{\mathrm{s}}\right\|_{\mathrm{v}, h}^{2}
\end{aligned}
$$

for all $\boldsymbol{v}_{h}^{\mathrm{s}} \in \boldsymbol{V}_{h}^{\mathrm{s}}$.

The next result states a fundamental property of the generalized Robin-Neumann schemes that will be useful for the stability analysis of Section 4.2. 
Lemma 2 Let $\left\{\left(\boldsymbol{u}_{h}^{n}, p_{h}^{n}, \boldsymbol{d}_{h}^{n}, \dot{\boldsymbol{d}}_{h}^{n}\right)\right\}_{n>r+1}$ be the sequence given by (17)-(18). For $n \geq r+1$, there holds

$$
\boldsymbol{u}_{h}^{n}=\dot{\boldsymbol{d}}_{h}^{n}+\frac{\tau}{\rho^{\mathrm{s}}}\left(\boldsymbol{L}_{h}^{\mathrm{e}}\left(\boldsymbol{d}_{h}^{n}-\boldsymbol{d}_{h}^{\star}\right)+\boldsymbol{L}_{h}^{\mathrm{v}}\left(\dot{\boldsymbol{d}}_{h}^{n}-\dot{\boldsymbol{d}}_{h}^{\star}\right)\right) \quad \text { on } \quad \Sigma
$$

and

$$
\begin{aligned}
\rho^{\mathrm{f}}\left(\partial_{\tau} \boldsymbol{u}_{h}^{n}, \boldsymbol{v}_{h}^{\mathrm{f}}\right)_{\Omega^{\mathrm{f}}}+a\left(\boldsymbol{u}_{h}^{n}, \boldsymbol{v}_{h}^{\mathrm{f}}\right)+b\left(p_{h}^{n}, \boldsymbol{v}_{h}^{\mathrm{f}}\right)-b\left(q_{h}, \boldsymbol{u}_{h}^{n}\right)+s_{h}\left(p_{h}^{n}, q_{h}\right) \\
+\rho^{\mathrm{s}}\left(\partial_{t} \dot{\boldsymbol{d}}_{h}^{n}, \boldsymbol{v}_{h}^{\mathrm{s}}\right)_{\Omega^{\mathrm{s}}, h}+a_{h}^{\mathrm{e}}\left(\boldsymbol{d}_{h}^{n}, \boldsymbol{v}_{h}^{\mathrm{s}}\right)+a_{h}^{\mathrm{v}}\left(\dot{\boldsymbol{d}}_{h}^{n}, \boldsymbol{v}_{h}^{\mathrm{s}}\right)=l\left(\boldsymbol{v}_{h}^{\mathrm{f}}\right)
\end{aligned}
$$

for all $\left(\boldsymbol{v}_{h}^{\mathrm{f}}, \boldsymbol{v}_{h}^{\mathrm{s}}\right) \in \boldsymbol{W}_{h}$ and $q_{h} \in Q_{h}$.

Proof 2 Due to (12), the fluid step (17) can be reformulated as

$$
\begin{aligned}
\rho^{\mathrm{f}}\left(\partial_{\tau} \boldsymbol{u}_{h}^{n}, \boldsymbol{v}_{h}^{\mathrm{f}}\right)_{\Omega^{\mathrm{f}}}+a\left(\boldsymbol{u}_{h}^{n}, \boldsymbol{v}_{h}^{\mathrm{f}}\right)+b\left(p_{h}^{n}, \boldsymbol{v}_{h}^{\mathrm{f}}\right)-b\left(q_{h}, \boldsymbol{u}_{h}^{n}\right)+s_{h}\left(p_{h}^{n}, q_{h}\right)+\frac{\rho^{\mathrm{s}}}{\tau}\left(\boldsymbol{B}_{h} \boldsymbol{u}_{h}^{n}, \boldsymbol{v}_{h}^{\mathrm{f}}\right)_{\Sigma} \\
=\frac{\rho^{\mathrm{s}}}{\tau}\left(\boldsymbol{B}_{h} \dot{\boldsymbol{d}}_{h}^{n-1}, \boldsymbol{v}_{h}^{\mathrm{f}}\right)_{\Sigma}+a_{h}^{\mathrm{e}}\left(\boldsymbol{d}_{h}^{\star}, \mathcal{L}_{h}^{\mathrm{s}} \boldsymbol{v}_{h}^{\mathrm{f}}\right)+a_{h}^{\mathrm{v}}\left(\dot{\boldsymbol{d}}_{h}^{\star}, \mathcal{L}_{h}^{\mathrm{s}} \boldsymbol{v}_{h}^{\mathrm{f}}\right)+l\left(\boldsymbol{v}_{h}^{\mathrm{f}}\right) .
\end{aligned}
$$

for all $\left(\boldsymbol{v}_{h}^{\mathrm{f}}, q_{h}\right) \in \boldsymbol{V}_{h}^{\mathrm{f}} \times Q_{h}$ and $n \geq r+1$. Furthermore, by testing $(18)_{2}$ with $\boldsymbol{v}_{h}^{\mathrm{s}}=\mathcal{L}_{h}^{\mathrm{s}} \boldsymbol{\xi}_{h}$ (for $\left.\boldsymbol{\xi}_{h} \in \boldsymbol{\Lambda}_{\Sigma, h}\right)$ and using (8), we infer that

$$
\begin{aligned}
\rho^{\mathrm{s}}\left(\boldsymbol{B}_{h} \partial_{\tau} \dot{\boldsymbol{d}}_{h}^{n}, \boldsymbol{\xi}_{h}\right)_{\Sigma}+a_{h}^{\mathrm{e}}\left(\boldsymbol{d}_{h}^{n}, \mathcal{L}_{h}^{\mathrm{s}} \boldsymbol{\xi}_{h}\right)+a_{h}^{\mathrm{v}}\left(\dot{\boldsymbol{d}}_{h}^{n}, \mathcal{L}_{h}^{\mathrm{s}} \boldsymbol{\xi}_{h}\right) \\
\quad=-\rho^{\mathrm{f}}\left(\partial_{\tau} \boldsymbol{u}_{h}^{n}, \mathcal{L}_{h}^{\mathrm{f}} \boldsymbol{\xi}_{h}\right)_{\Omega^{\mathrm{f}}}-a\left(\boldsymbol{u}_{h}^{n}, \mathcal{L}_{h}^{\mathrm{f}} \boldsymbol{\xi}_{h}\right)-b\left(p_{h}^{n}, \mathcal{L}_{h}^{\mathrm{f}} \boldsymbol{\xi}_{h}\right) .
\end{aligned}
$$

Hence, taking $\left(\boldsymbol{v}_{h}^{\mathrm{f}}, q_{h}\right)=\left(\mathcal{L}_{h}^{\mathrm{f}} \boldsymbol{\xi}_{h}, 0\right)$ in (32) and subtracting the resulting expression from (33) yields

$$
\frac{\rho^{\mathrm{s}}}{\tau}\left(\boldsymbol{B}_{h}\left(\dot{\boldsymbol{d}}_{h}^{n}-\boldsymbol{u}_{h}^{n}\right), \boldsymbol{\xi}_{h}\right)_{\Sigma}+a^{\mathrm{e}}\left(\boldsymbol{d}_{h}^{n}-\boldsymbol{d}_{h}^{\star}, \mathcal{L}_{h}^{\mathrm{s}} \boldsymbol{\xi}_{h}\right)+a^{\mathrm{v}}\left(\dot{\boldsymbol{d}}_{h}^{n}-\dot{\boldsymbol{d}}_{h}^{\star}, \mathcal{L}_{h}^{\mathrm{s}} \boldsymbol{\xi}_{h}\right)=0
$$

for all $\boldsymbol{\xi}_{h} \in \boldsymbol{\Lambda}_{\Sigma, h}$ and $n \geq r+1$. Equivalently, from (28) and (8), we have

$$
\frac{\rho^{\mathrm{s}}}{\tau} \boldsymbol{B}_{h}\left(\dot{\boldsymbol{d}}_{h}^{n}-\boldsymbol{u}_{h}^{n}\right)+\boldsymbol{B}_{h} \boldsymbol{L}_{h}^{\mathrm{e}}\left(\boldsymbol{d}_{h}^{n}-\boldsymbol{d}_{h}^{\star}\right)+\boldsymbol{B}_{h} \boldsymbol{L}_{h}^{\mathrm{v}}\left(\dot{\boldsymbol{d}}_{h}^{n}-\dot{\boldsymbol{d}}_{h}^{\star}\right)=\mathbf{0} \quad \text { on } \quad \Sigma
$$

for $n \geq r+1$. The identity (30) then results from the invertibility of the interface operator $\boldsymbol{B}_{h}$.

At last, the relation (31) follows from (17) with $\boldsymbol{v}^{\mathrm{f}}=\widetilde{\boldsymbol{v}}^{\mathrm{f}} \in \boldsymbol{V}_{\Sigma, h}^{\mathrm{f}}$ and adding the resulting expression to (18). This concludes the proof. $\diamond$

Lemma 2 shows that the explicit coupling schemes (17)-(18) are kinematic perturbations of the implicit coupling scheme (23). Note that, owing to (30), we do not have $\left(\boldsymbol{u}_{h}^{n}, \dot{d}_{h}^{n}\right) \in \boldsymbol{W}_{h}$ in general. Note that the size of the perturbation (and hence accuracy) depends on the time-step length, the discrete solid operators and the extrapolations of the solid displacement and velocity. In the next section, the stability of (17)-(18) is analyzed by investigating the impact of the perturbed kinematic constraint (30) on the stability of the underlying implicit coupling scheme.

By applying to (24)-(25) the same arguments than in the proof of Lemma 2, we can state the following result, which will be useful for the convergence analysis of Section 4.3. 
Lemma 3 Let $\left\{\left(\boldsymbol{u}_{h, k}, p_{h, k}, \boldsymbol{d}_{h, k}, \dot{\boldsymbol{d}}_{h, k}\right)\right\}_{k \geq 1}$ be the sequence of approximations given by (24)-(25). Then, for $k \geq 1$, there holds

$$
\left\{\begin{array}{l}
\boldsymbol{u}_{h, k}=\dot{\boldsymbol{d}}_{h, k}+\frac{\tau}{\rho^{\mathrm{s}}}\left(\boldsymbol{L}_{h}^{\mathrm{e}}\left(\boldsymbol{d}_{h, k}-\boldsymbol{d}_{h, k-1}\right)+\boldsymbol{L}_{h}^{\mathrm{v}}\left(\dot{\boldsymbol{d}}_{h, k}-\dot{\boldsymbol{d}}_{h, k-1}\right)\right) \quad \text { on } \quad \Sigma, \\
\dot{\boldsymbol{d}}_{h, k}=\frac{\boldsymbol{d}_{h, k}-\boldsymbol{d}_{h}^{n-1}}{\tau} \text { in } \Omega^{\mathrm{s}}, \\
\frac{\rho^{\mathrm{f}}}{\tau}\left(\boldsymbol{u}_{h, k}-\boldsymbol{u}_{h}^{n-1}, \boldsymbol{v}_{h}^{\mathrm{f}}\right)_{\Omega^{\mathrm{f}}}+a\left(\boldsymbol{u}_{h, k}, \boldsymbol{v}_{h}^{\mathrm{f}}\right)+b\left(p_{h, k}, \boldsymbol{v}_{h}^{\mathrm{f}}\right)-b\left(q_{h}, \boldsymbol{u}_{h, k}\right)+s_{h}\left(p_{h, k}, q_{h}\right) \\
\quad+\frac{\rho^{\mathrm{s}}}{\tau}\left(\dot{\boldsymbol{d}}_{h, k}-\dot{\boldsymbol{d}}_{h}^{n-1}, \boldsymbol{v}_{h}^{\mathrm{s}}\right)_{\Omega^{\mathrm{s}}, h}+a_{h}^{\mathrm{e}}\left(\boldsymbol{d}_{h, k}, \boldsymbol{v}_{h}^{\mathrm{s}}\right)+a_{h}^{\mathrm{v}}\left(\dot{\boldsymbol{d}}_{h, k}, \boldsymbol{v}_{h}^{\mathrm{s}}\right)=l\left(\boldsymbol{v}_{h}^{\mathrm{f}}\right)
\end{array}\right.
$$

for all $\left(\boldsymbol{v}_{h}^{\mathrm{f}}, \boldsymbol{v}_{h}^{\mathrm{s}}\right) \in \boldsymbol{W}_{h}$ and $q_{h} \in Q_{h}$.

\subsection{Stability analysis of the explicit coupling schemes}

For $n \geq 0$, we define the discrete energy of the fluid-structure system, at time $t_{n}$, as

$$
E_{h}^{n} \stackrel{\text { def }}{=} \frac{\rho^{\mathrm{f}}}{2}\left\|\boldsymbol{u}_{h}^{n}\right\|_{0, \Omega^{\mathrm{f}}}^{2}+\frac{\rho^{\mathrm{s}}}{2}\left\|\dot{\boldsymbol{d}}_{h}^{n}\right\|_{0, \Omega^{\mathrm{s}}}^{2}+\frac{1}{2}\left\|\boldsymbol{d}_{h}^{n}\right\|_{\mathrm{e}}^{2}
$$

and, for $n \geq 1$, the total dissipation as

$$
\begin{aligned}
D_{h}^{n} \stackrel{\text { def }}{=} \frac{1}{2}\left(\rho^{\mathrm{f}}\left\|\boldsymbol{u}_{h}^{n}-\boldsymbol{u}_{h}^{n-1}\right\|_{0, \Omega^{\mathrm{f}}}^{2}+\rho^{\mathrm{s}}\left\|\dot{\boldsymbol{d}}_{h}^{n}-\dot{\boldsymbol{d}}_{h}^{n-1}\right\|_{0, \Omega^{\mathrm{s}}}^{2}\right. & \left.+\left\|\boldsymbol{d}_{h}^{n}-\boldsymbol{d}_{h}^{n-1}\right\|_{\mathrm{e}}^{2}\right) \\
& +2 \mu \tau\left\|\boldsymbol{\epsilon}\left(\boldsymbol{u}_{h}^{n}\right)\right\|_{0, \Omega^{\mathrm{f}}}^{2}+\tau\left|p_{h}^{n}\right|_{s_{h}}^{2}+\tau\left\|\dot{\boldsymbol{d}}_{h}^{n}\right\|_{\mathrm{v}}^{2},
\end{aligned}
$$

where $\left|p_{h}^{n}\right|_{s_{h}} \stackrel{\text { def }}{=}\left(s_{h}\left(p_{h}^{n}, p_{h}^{n}\right)\right)^{\frac{1}{2}}$. The following result states the energy stability of the explicit coupling schemes given by (17)-(18).

Theorem 4 Assume that $\boldsymbol{f}^{\Gamma}=\mathbf{0}$ (free system) and let $\left\{\left(\boldsymbol{u}_{h}^{n}, p_{h}^{n}, \boldsymbol{d}_{h}^{n}, \dot{\boldsymbol{d}}_{h}^{n}\right)\right\}_{n \geq r+1}$ be the sequence given by (17)-(18). The following a priori energy estimates hold:

- Schemes with $r=0$ or $r=1$ :

$$
E_{h}^{n}+\sum_{m=r+1}^{n} D_{h}^{m} \lesssim E_{h}^{0}
$$

for $n \geq r+1$.

- Scheme with $r=2$ :

$$
E_{h}^{n}+\sum_{m=3}^{n} D_{h}^{m} \lesssim \exp \left(\frac{t_{n} \gamma}{1-\gamma \tau}\right) E_{h}^{0}
$$

for $n \geq 3$, provided that the following conditions hold

$$
\left\{\begin{aligned}
\tau\left(\alpha+\beta\left(\frac{\omega_{\mathrm{e}}}{h}\right)^{2}\right) & <\delta, \\
\tau^{5}\left(\frac{\omega_{\mathrm{e}}}{h}\right)^{6}+\tau^{2}\left(\frac{\omega_{\mathrm{e}}}{h}\right)^{2}\left(\alpha+\beta\left(\frac{\omega_{\mathrm{e}}}{h}\right)^{2}\right) & <\gamma, \\
\tau \gamma & <1,
\end{aligned}\right.
$$

where $\omega_{\mathrm{e}} \stackrel{\text { def }}{=} C_{\text {inv }} \sqrt{\beta_{\mathrm{e}} / \rho^{\mathrm{s}}}, 0 \leq \delta \leq 1$ and $\gamma>0$. 
Proof 3 The proof is based on the generalization of the arguments used in [13, 10]. We denote by $\boldsymbol{w}_{h}^{n}$ the quantity given by

$$
\boldsymbol{w}_{h}^{n} \stackrel{\text { def }}{=} \dot{\boldsymbol{d}}_{h}^{n}+\frac{\tau}{\rho^{\mathrm{s}}}\left(\boldsymbol{L}_{h}^{\mathrm{e}}\left(\boldsymbol{d}_{h}^{n}-\boldsymbol{d}_{h}^{\star}\right)+\boldsymbol{L}_{h}^{\mathrm{v}}\left(\dot{\boldsymbol{d}}_{h}^{n}-\dot{\boldsymbol{d}}_{h}^{\star}\right)\right) .
$$

Owing to (30), we have $\left.\boldsymbol{w}_{h}^{n}\right|_{\Sigma}=\left.\boldsymbol{u}_{h}^{n}\right|_{\Sigma}$. Thus, we can take $\left(\boldsymbol{v}_{h}^{\mathrm{f}}, \boldsymbol{v}_{h}^{\mathrm{s}}\right)=\tau\left(\boldsymbol{u}_{h}^{n}, \boldsymbol{w}_{h}^{n}\right)$ and $q_{h}=\tau p_{h}^{n}$ in (31), which yields

$$
\begin{aligned}
\frac{\rho^{\mathrm{f}}}{2}\left(\left\|\boldsymbol{u}_{h}^{n}\right\|_{0, \Omega^{\mathrm{f}}}^{2}-\left\|\boldsymbol{u}_{h}^{n-1}\right\|_{0, \Omega^{\mathrm{f}}}^{2}+\| \boldsymbol{u}_{h}^{n}-\right. & \left.\boldsymbol{u}_{h}^{n-1} \|_{0, \Omega^{\mathrm{f}}}^{2}\right)+2 \mu \tau\left\|\boldsymbol{\epsilon}\left(\boldsymbol{u}_{h}^{n}\right)\right\|_{0, \Omega^{\mathrm{f}}}^{2}+\tau\left|p_{h}^{n}\right|_{\mathrm{s}, h}^{2} \\
& +\tau \rho^{\mathrm{s}}\left(\partial_{\tau} \dot{\boldsymbol{d}}_{h}^{n}, \boldsymbol{w}_{h}^{n}\right)_{\Omega^{\mathrm{s}}, h}+\tau a_{h}^{\mathrm{e}}\left(\boldsymbol{d}_{h}^{n}, \boldsymbol{w}_{h}^{n}\right)+\tau a_{h}^{\mathrm{v}}\left(\dot{\boldsymbol{d}}_{h}^{n}, \boldsymbol{w}_{h}^{n}\right)=0 .
\end{aligned}
$$

Furthermore, by inserting (38) in this equality, using (28) and Remark 5, we get

$$
\begin{gathered}
E_{h}^{n}-E_{h}^{n-1}+D_{h}^{n}+\underbrace{\tau\left(\dot{\boldsymbol{d}}_{h}^{n}-\dot{\boldsymbol{d}}_{h}^{n-1}, \boldsymbol{L}_{h}^{\mathrm{e}}\left(\boldsymbol{d}_{h}^{n}-\boldsymbol{d}_{h}^{\star}\right)+\boldsymbol{L}_{h}^{\mathrm{v}}\left(\dot{\boldsymbol{d}}_{h}^{n}-\dot{\boldsymbol{d}}_{h}^{\star}\right)\right)_{\Omega^{\mathrm{s}}, h}}_{I_{1}} \\
+\underbrace{\frac{\tau^{2}}{\rho^{\mathrm{s}}}\left(\boldsymbol{L}_{h}^{\mathrm{e}} \boldsymbol{d}_{h}^{n}+\boldsymbol{L}_{h}^{\mathrm{v}} \dot{\boldsymbol{d}}_{h}^{n}, \boldsymbol{L}_{h}^{\mathrm{e}}\left(\boldsymbol{d}_{h}^{n}-\boldsymbol{d}_{h}^{\star}\right)+\boldsymbol{L}_{h}^{\mathrm{v}}\left(\dot{\boldsymbol{d}}_{h}^{n}-\dot{\boldsymbol{d}}_{h}^{\star}\right)\right)_{\Omega^{\mathrm{s}}, h}}_{I_{2}} \lesssim 0 .
\end{gathered}
$$

Therefore, it only remains to control the terms $I_{1}$ and $I_{2}$. We proceed by treating each case separately, depending on the extrapolation order $r$.

(i) Scheme with $r=0$. We have

$$
\begin{aligned}
& I_{1} \geq-\frac{3 \tau^{2}}{4 \rho^{\mathrm{s}}}\left\|\boldsymbol{L}_{h}^{\mathrm{e}} \boldsymbol{d}_{h}^{n}+\boldsymbol{L}_{h}^{\mathrm{v}} \dot{\boldsymbol{d}}_{h}^{n}\right\|_{\mathrm{s}, h}^{2}-\frac{\rho^{\mathrm{s}}}{3}\left\|\dot{\boldsymbol{d}}_{h}^{n}-\dot{\boldsymbol{d}}_{h}^{n-1}\right\|_{\mathrm{s}, h}^{2}, \\
& I_{2}=\frac{\tau^{2}}{\rho^{\mathrm{s}}}\left\|\boldsymbol{L}_{h}^{\mathrm{e}} \boldsymbol{d}_{h}^{n}+\boldsymbol{L}_{h}^{\mathrm{v}} \dot{\boldsymbol{d}}_{h}^{n}\right\|_{\mathrm{s}, h}^{2}
\end{aligned}
$$

for $n \geq 1$. Hence, by inserting these estimates into (39) and summing over $m=1, \ldots, n$, we get

$$
E_{h}^{n}+\sum_{m=1}^{n}\left(D_{h}^{m}+D_{0, \mathrm{spl}}^{m}\right) \lesssim E_{h}^{0}
$$

for $n \geq 1$, and with the additional dissipation related to the splitting

$$
D_{0, \mathrm{spl}}^{m} \stackrel{\text { def }}{=} \frac{\tau^{2}}{\rho^{\mathrm{s}}}\left\|\boldsymbol{L}_{h}^{\mathrm{e}} \boldsymbol{d}_{h}^{m}+\boldsymbol{L}_{h}^{\mathrm{v}} \dot{\boldsymbol{d}}_{h}^{m}\right\|_{\mathrm{s}, h}^{2} .
$$

The estimate (35) with $r=0$ follows from (40).

(ii) Scheme with $r=1$. In this case, we have

$$
\begin{aligned}
I_{1} & =\frac{\tau^{2}}{2}\left(\left\|\dot{\boldsymbol{d}}_{h}^{n}\right\|_{\mathrm{e}, h}^{2}-\left\|\dot{\boldsymbol{d}}_{h}^{n-1}\right\|_{\mathrm{e}, h}^{2}+\left\|\dot{\boldsymbol{d}}_{h}^{n}-\dot{\boldsymbol{d}}_{h}^{n-1}\right\|_{\mathrm{e}, h}^{2}\right)+\tau\left\|\dot{\boldsymbol{d}}_{h}^{n}-\dot{\boldsymbol{d}}_{h}^{n-1}\right\|_{\mathrm{v}, h}^{2}, \\
I_{2} & =\frac{\tau^{2}}{2 \rho^{\mathrm{s}}}\left(\left\|\boldsymbol{L}_{h}^{\mathrm{e}} \boldsymbol{d}_{h}^{n}+\boldsymbol{L}_{h}^{\mathrm{v}} \dot{\boldsymbol{d}}_{h}^{n}\right\|_{\mathrm{s}, h}^{2}-\left\|\boldsymbol{L}_{h}^{\mathrm{e}} \boldsymbol{d}_{h}^{n-1}+\boldsymbol{L}_{h}^{\mathrm{v}} \dot{\boldsymbol{d}}_{h}^{n-1}\right\|_{\mathrm{s}, h}^{2}\right) .
\end{aligned}
$$

Hence, from (39), we infer that

$$
E_{h}^{n}+E_{1, \mathrm{spl}}^{n}+\sum_{m=2}^{n}\left(D_{h}^{m}+D_{1, \mathrm{spl}}^{m}\right) \lesssim E_{h}^{1}+D_{h}^{1}+D_{0, \mathrm{spl}}^{1}
$$

$\mathrm{RR} \mathrm{n}^{\circ} 8384$ 
for $n \geq 2$, and with the additional energy and dissipation introduced by the splitting

$$
\begin{aligned}
& E_{1, \mathrm{spl}}^{n} \stackrel{\text { def }}{=} \tau^{2}\left\|\dot{\boldsymbol{d}}_{h}^{n}\right\|_{\mathrm{e}, h}^{2}+\frac{\tau^{2}}{\rho^{\mathrm{s}}}\left\|\boldsymbol{L}_{h}^{\mathrm{e}} \boldsymbol{d}_{h}^{n}+\boldsymbol{L}_{h}^{\mathrm{v}} \dot{\boldsymbol{d}}_{h}^{n}\right\|_{\mathrm{s}, h}^{2}, \\
& D_{1, \mathrm{spl}}^{n} \stackrel{\text { def }}{=} \tau^{2}\left\|\dot{\boldsymbol{d}}_{h}^{n}-\dot{\boldsymbol{d}}_{h}^{n-1}\right\|_{\mathrm{e}, h}^{2}+\tau\left\|\dot{\boldsymbol{d}}_{h}^{n}-\dot{\boldsymbol{d}}_{h}^{n-1}\right\|_{\mathrm{v}, h}^{2} .
\end{aligned}
$$

Due to the initialization procedure, the estimate (35) for $r=1$ results from (41) and (40) with $n=1$.

(iii) Scheme with $r=2$. For the first term, We have

$$
\begin{aligned}
I_{1}= & \tau^{2}\left\|\dot{\boldsymbol{d}}_{h}^{n}-\dot{\boldsymbol{d}}_{h}^{n-1}\right\|_{\mathrm{e}, h}^{2} \\
& +\frac{\tau}{2}\left(\left\|\dot{\boldsymbol{d}}_{h}^{n}-\dot{\boldsymbol{d}}_{h}^{n-1}\right\|_{\mathrm{v}, h}^{2}-\left\|\dot{\boldsymbol{d}}_{h}^{n-1}-\dot{\boldsymbol{d}}_{h}^{n-2}\right\|_{\mathrm{v}, h}^{2}+\left\|\dot{\boldsymbol{d}}_{h}^{n}-2 \dot{\boldsymbol{d}}_{h}^{n-1}+\dot{\boldsymbol{d}}_{h}^{n-2}\right\|_{\mathrm{v}, h}^{2}\right)
\end{aligned}
$$

for $n \geq 3$. The term $I_{2}$ is split into three parts that we estimate separately:

$$
\begin{aligned}
I_{2}= & \underbrace{\frac{\tau^{3}}{\rho^{\mathrm{s}}} a_{h}^{\mathrm{e}}\left(\boldsymbol{L}_{h}^{\mathrm{e}} \boldsymbol{d}_{h}^{n}, \dot{\boldsymbol{d}}_{h}^{n}-\dot{\boldsymbol{d}}_{h}^{n-1}\right)}_{J_{1}}+\underbrace{\frac{\tau^{3}}{\rho^{\mathrm{s}}}\left(\boldsymbol{L}_{h}^{\mathrm{v}} \dot{\boldsymbol{d}}_{h}^{n}, \boldsymbol{L}_{h}^{\mathrm{e}}\left(\dot{\boldsymbol{d}}_{h}^{n}-\dot{\boldsymbol{d}}_{h}^{n-1}\right)\right)_{\mathrm{s}, h}}_{J_{2}} \\
& +\underbrace{\frac{\tau^{2}}{\rho^{\mathrm{s}}} a_{h}^{\mathrm{v}}\left(\boldsymbol{L}_{h}^{\mathrm{e}} \boldsymbol{d}_{h}^{n}+\boldsymbol{L}_{h}^{\mathrm{v}} \dot{\boldsymbol{d}}_{h}^{n}, \dot{\boldsymbol{d}}_{h}^{n}-2 \dot{\boldsymbol{d}}_{h}^{n-1}+\dot{\boldsymbol{d}}_{h}^{n-2}\right)}_{J_{3}}
\end{aligned}
$$

The first term is estimated, using (29), as follows

$$
\begin{aligned}
J_{1} & \geq-\frac{\tau^{3}}{\rho^{\mathrm{s}}}\left\|\boldsymbol{L}_{h}^{\mathrm{e}} \boldsymbol{d}_{h}^{n}\right\|_{\mathrm{e}, h}\left\|\dot{\boldsymbol{d}}_{h}^{n}-\dot{\boldsymbol{d}}_{h}^{n-1}\right\|_{\mathrm{e}, h} \geq-\frac{\tau^{3}}{\rho^{\mathrm{s}}} \frac{\beta_{\mathrm{e}}^{\frac{3}{2}} C_{\mathrm{inv}}^{3}}{h^{3}}\left\|\boldsymbol{d}_{h}^{n}\right\|_{\mathrm{e}, h}\left\|\dot{\boldsymbol{d}}_{h}^{n}-\dot{\boldsymbol{d}}_{h}^{n-1}\right\|_{\mathrm{s}, h} \\
& \geq-\frac{\tau^{6} \omega_{\mathrm{e}}^{6}}{h^{6}}\left\|\boldsymbol{d}_{h}^{n}\right\|_{\mathrm{e}, h}^{2}-\frac{\rho^{\mathrm{s}}}{4}\left\|\dot{\boldsymbol{d}}_{h}^{n}-\dot{\boldsymbol{d}}_{h}^{n-1}\right\|_{\mathrm{s}, h}^{2}
\end{aligned}
$$

Owing to the particular expression of the Rayleigh damping, the second term yields the following telescoping series

$$
\begin{aligned}
J_{2}= & \frac{\alpha \rho^{\mathrm{s}} \tau^{3}}{2}\left(\left\|\dot{\boldsymbol{d}}_{h}^{n}\right\|_{\mathrm{e}, h}^{2}-\left\|\dot{\boldsymbol{d}}_{h}^{n-1}\right\|_{\mathrm{e}, h}^{2}+\left\|\dot{\boldsymbol{d}}_{h}^{n}-\dot{\boldsymbol{d}}_{h}^{n-1}\right\|_{\mathrm{e}, h}^{2}\right) \\
& +\frac{\beta \tau^{3}}{2 \rho^{\mathrm{s}}}\left(\left\|\boldsymbol{L}_{h}^{\mathrm{e}} \dot{\boldsymbol{d}}_{h}^{n}\right\|_{\mathrm{s}, h}^{2}-\left\|\boldsymbol{L}_{h}^{\mathrm{e}} \dot{\boldsymbol{d}}_{h}^{n-1}\right\|_{\mathrm{s}, h}^{2}+\left\|\boldsymbol{L}_{h}^{\mathrm{e}}\left(\dot{\boldsymbol{d}}_{h}^{n}-\dot{\boldsymbol{d}}_{h}^{n-1}\right)\right\|_{\mathrm{s}, h}^{2}\right) .
\end{aligned}
$$

At last, using (29) once more, for the third term we get

$$
\begin{aligned}
J_{3} \geq & -\frac{\tau^{3}}{\left(\rho^{\mathrm{s}}\right)^{2}}\left\|\boldsymbol{L}_{h}^{\mathrm{e}} \boldsymbol{d}_{h}^{n}\right\|_{\mathrm{v}, h}^{2}-\frac{\tau^{3}}{\left(\rho^{\mathrm{s}}\right)^{2}}\left\|\boldsymbol{L}_{h}^{\mathrm{v}} \dot{\boldsymbol{d}}_{h}^{n}\right\|_{\mathrm{v}, h}^{2}-\frac{\tau}{4}\left\|\dot{\boldsymbol{d}}_{h}^{n}-2 \dot{\boldsymbol{d}}_{h}^{n-1}+\dot{\boldsymbol{d}}_{h}^{n-2}\right\|_{\mathrm{v}, h}^{2} \\
\geq & -\frac{\tau^{3} \omega_{\mathrm{e}}^{2}}{h^{2}}\left(\alpha+\beta \frac{\omega_{\mathrm{e}}^{2}}{h^{2}}\right)\left\|\boldsymbol{d}_{h}^{n}\right\|_{\mathrm{e}, h}^{2}-\tau^{3}\left(\alpha+\beta \frac{\omega_{\mathrm{e}}^{2}}{h^{2}}\right)^{2}\left\|\dot{\boldsymbol{d}}_{h}^{n}\right\|_{\mathrm{v}, h}^{2} \\
& -\frac{\tau}{2}\left\|\dot{\boldsymbol{d}}_{h}^{n}-2 \dot{\boldsymbol{d}}_{h}^{n-1}+\dot{\boldsymbol{d}}_{h}^{n-2}\right\|_{\mathrm{v}, h}^{2} .
\end{aligned}
$$

By summing over $m=1, \ldots, n$ and by applying the discrete Gronwall lemma, under conditions 
(37), we get the following bound, for $n \geq 3$,

$$
\begin{aligned}
E_{h}^{n}+\sum_{m=3}^{n} D_{h}^{m} & \\
& \lesssim e^{\left(\frac{t_{n} \gamma}{1-\gamma \tau}\right)}\left(E_{h}^{2}+\tau\left\|\dot{\boldsymbol{d}}_{h}^{2}-\dot{\boldsymbol{d}}_{h}^{1}\right\|_{\mathrm{v}, h}^{2}+\alpha \tau^{3}\left\|\dot{\boldsymbol{d}}_{h}^{2}\right\|_{\mathrm{e}, h}^{2}+\frac{\beta \tau^{3}}{\rho^{\mathrm{s}}}\left\|\boldsymbol{L}_{h}^{\mathrm{e}} \dot{\boldsymbol{d}}_{h}^{2}\right\|_{\mathrm{s}, h}^{2}\right) .
\end{aligned}
$$

The estimate (36) follows from (43), whose right-hand side can be bounded using the energy estimate (40) of the scheme with $r=1$, and the sability condition (37). More precisely, we clearly have $E_{h}^{2} \leq E_{h}^{0}, \tau\left\|\dot{\boldsymbol{d}}_{h}^{2}-\dot{\boldsymbol{d}}_{h}^{1}\right\|_{\mathrm{v}, h}^{2} \leq D_{1, \mathrm{spl}}^{2}$, and the stability condition and (29) yield

$$
\begin{aligned}
\alpha \tau^{3}\left\|\dot{\boldsymbol{d}}_{h}^{2}\right\|_{\mathrm{e}, h}^{2}+\frac{\beta \tau^{3}}{\rho^{\mathrm{s}}}\left\|\boldsymbol{L}_{h}^{\mathrm{e}} \dot{\boldsymbol{d}}_{h}^{2}\right\|_{\mathrm{s}, h}^{2} & \leq \tau^{3}\left(\alpha+\beta\left(\frac{\omega_{\mathrm{e}}}{h}\right)^{2}\right)\left\|\dot{\boldsymbol{d}}_{h}^{2}\right\|_{\mathrm{e}, h}^{2} \\
& \leq \delta \tau^{3}\left\|\dot{\boldsymbol{d}}_{h}^{2}\right\|_{\mathrm{e}, h}^{2} \lesssim E_{1, \mathrm{spl}}^{2} .
\end{aligned}
$$

Hence, the proof is complete. $\diamond$

We conclude this section with a series of obvservations. Theorem 4 guarantees the addedmass free stability of the generalized Robin-Neumann schemes (17)-(18). Unconditional stability is obtained for $r=0$ and $r=1$. Note that, in these cases, the results are independent of the structure of the solid viscous bilinear-form $a_{h}^{\mathrm{v}}$. In fact, only symmetry and positiveness are necessary. Therefore, the estimate (35) remains valid if, instead of $a_{h}^{\mathrm{v}}$, we consider the original bilinear-form $a^{\mathrm{v}}$ in (17)-(18), without mass-lumping approximation in the zeroth-order term.

Theorem 4 shows also that the scheme with second-order extrapolation $(r=2)$ and without solid damping $(\alpha=\beta=0)$ is conditionally stable under a $6 / 5$-CFL condition $\tau=\mathcal{O}\left(h^{6 / 5}\right)$. If solid damping effects are present, additional conditions are required. In particular, for $\beta \neq 0$, stability is guaranteed under a parabolic-CFL condition $\tau=\mathcal{O}\left(h^{2}\right)$, which enforces much more restrictive conditions on the discretization parameters.

Remark 6 Similar estimates were obtained in [13, Theorem 1] for the original Robin-Neumann schemes, in the case of the coupling with thin-walled structures. This shows that the extension proposed in this work preserve their stability properties.

\subsection{Convergence of the iterative solution procedure}

This section is devoted to the convergence analysis of the iterative solution procedure (24)-(25) towards the implicit coupling solution (23). The main result is stated in the next theorem.

Theorem 5 For $n \geq 1$, let $\left(\boldsymbol{u}_{h}^{n}, p_{h}^{n}, \boldsymbol{d}_{h}^{n}, \dot{\boldsymbol{d}}_{h}^{n}\right)$ be given by the implicit scheme (23) and $\left\{\left(\boldsymbol{u}_{h, k}, p_{h, k}, \boldsymbol{d}_{h, k}, \dot{\boldsymbol{d}}_{h, k}\right)\right\}_{k \geq 1}$ be the sequence of approximations given by (24)-(25). Then, there holds

$$
\begin{aligned}
& \sum_{k=1}^{\infty}\left(\rho^{\mathrm{f}}\left\|\boldsymbol{u}_{h}^{n}-\boldsymbol{u}_{h, k}\right\|_{0, \Omega^{\mathrm{f}}}^{2}+\rho^{\mathrm{s}}\left\|\dot{\boldsymbol{d}}_{h}^{n}-\dot{\boldsymbol{d}}_{h, k}\right\|_{0, \Omega^{\mathrm{s}}}^{2}+\left\|\boldsymbol{d}_{h}^{n}-\boldsymbol{d}_{h, k}\right\|_{\mathrm{e}}^{2}\right) \\
& \lesssim\left\|\boldsymbol{d}_{h}^{n}-\boldsymbol{d}_{h, 0}\right\|_{\mathrm{e}}^{2}+\tau\left\|\dot{\boldsymbol{d}}_{h, 0}-\dot{\boldsymbol{d}}_{h}^{n}\right\|_{\mathrm{v}}^{2} \\
&+\frac{\tau^{2}}{\rho^{\mathrm{s}} \epsilon}\left\|\boldsymbol{L}_{h}^{\mathrm{e}}\left(\boldsymbol{d}_{h}^{n}-\boldsymbol{d}_{h, 0}\right)+\boldsymbol{L}_{h}^{\mathrm{v}}\left(\dot{\boldsymbol{d}}_{h}^{n}-\dot{\boldsymbol{d}}_{h, 0}\right)\right\|_{0, \Omega^{\mathrm{s}}}^{2} .
\end{aligned}
$$

In particular, we have

$$
\rho^{\mathrm{f}}\left\|\boldsymbol{u}_{h}^{n}-\boldsymbol{u}_{h, k}\right\|_{0, \Omega^{\mathrm{f}}}+\rho^{\mathrm{s}}\left\|\dot{\boldsymbol{d}}_{h}^{n}-\dot{\boldsymbol{d}}_{h, k}\right\|_{0, \Omega^{\mathrm{s}}}+\left\|\boldsymbol{d}_{h}^{n}-\boldsymbol{d}_{h, k}\right\|_{\mathrm{e}} \underset{k \rightarrow \infty}{\longrightarrow} 0
$$


Proof 4 We introduce the following errors between the $k$-th iteration of (24)-(25) and the $n$-th step of (23):

$$
\boldsymbol{e}_{h, k}^{\boldsymbol{u}} \stackrel{\text { def }}{=} \boldsymbol{u}_{h}^{n}-\boldsymbol{u}_{h, k}, \quad e_{h, k}^{p} \stackrel{\text { def }}{=} p_{h}^{n}-p_{h, k}, \quad \boldsymbol{e}_{h, k}^{\boldsymbol{d}} \stackrel{\text { def }}{=} \boldsymbol{d}_{h}^{n}-\boldsymbol{d}_{h, k}, \quad \boldsymbol{e}_{h, k}^{\dot{\boldsymbol{d}}} \stackrel{\text { def }}{=} \dot{\boldsymbol{d}}_{h}^{n}-\dot{\boldsymbol{d}}_{h, k} .
$$

Since $\left.\boldsymbol{u}_{h}^{n}\right|_{\Sigma}=\left.\dot{\boldsymbol{d}}_{h}^{n}\right|_{\Sigma}$, the subtraction of (34) from (23) yields the following error equation

$$
\left\{\begin{array}{l}
\boldsymbol{e}_{h, k}^{\boldsymbol{u}}=\boldsymbol{e}_{h, k}^{\dot{\boldsymbol{d}}}+\frac{\tau}{\rho^{\mathrm{s}}}\left(\boldsymbol{L}_{h}^{\mathrm{e}}\left(\boldsymbol{e}_{h, k}^{\boldsymbol{d}}-\boldsymbol{e}_{h, k-1}^{\boldsymbol{d}}\right)+\boldsymbol{L}_{h}^{\mathrm{v}}\left(\boldsymbol{e}_{h, k}^{\dot{\boldsymbol{d}}}-\boldsymbol{e}_{h, k-1}^{\dot{\boldsymbol{d}}}\right)\right) \quad \text { on } \Sigma, \\
\boldsymbol{e}_{h, k}^{\dot{\boldsymbol{d}}}=\frac{1}{\tau} \boldsymbol{e}_{h, k}^{\boldsymbol{d}} \text { in } \Omega^{\mathrm{s}}, \\
\frac{\rho^{\mathrm{f}}}{\tau}\left(\boldsymbol{e}_{h, k}^{\boldsymbol{u}}, \boldsymbol{v}_{h}^{\mathrm{f}}\right)_{\Omega^{\mathrm{f}}}+a\left(\boldsymbol{e}_{h, k}^{\boldsymbol{u}}, \boldsymbol{v}_{h}^{\mathrm{f}}\right)+b\left(e_{h, k}^{p}, \boldsymbol{v}_{h}^{\mathrm{f}}\right)-b\left(q_{h}, \boldsymbol{e}_{h, k}^{\boldsymbol{u}}\right)+s_{h}\left(e_{h, k}^{p}, q_{h}\right) \\
\quad+\frac{\rho^{\mathrm{s}}}{\tau}\left(\boldsymbol{e}_{h, k}^{\dot{\boldsymbol{d}}}, \boldsymbol{v}_{h}^{\mathrm{s}}\right)_{\mathrm{s}, h}+a_{h}^{\mathrm{e}}\left(\boldsymbol{e}_{h, k}^{\boldsymbol{d}}, \boldsymbol{v}_{h}^{\mathrm{s}}\right)+a_{h}^{\mathrm{v}}\left(\boldsymbol{e}_{h, k}^{\dot{\boldsymbol{d}}}, \boldsymbol{v}_{h}^{\mathrm{s}}\right)=0
\end{array}\right.
$$

for all $\left(\boldsymbol{v}_{h}^{\mathrm{f}}, \boldsymbol{v}_{h}^{\mathrm{s}}\right) \in \boldsymbol{W}_{h}$ and $q_{h} \in Q_{h}$.

We proceed by taking $\boldsymbol{v}_{h}^{\mathrm{f}}=\tau \boldsymbol{e}_{h, k}^{\boldsymbol{u}}, q_{h}=\tau e_{h, k}^{p}$ and

$$
\boldsymbol{v}_{h}^{\mathrm{s}}=\tau \boldsymbol{e}_{h, k}^{\dot{\boldsymbol{d}}}+\frac{\tau^{2}}{\rho^{\mathrm{s}}}\left(\boldsymbol{L}_{h}^{\mathrm{e}}\left(\boldsymbol{e}_{h, k}^{\boldsymbol{d}}-\boldsymbol{e}_{h, k-1}^{\boldsymbol{d}}\right)+\boldsymbol{L}_{h}^{\mathrm{v}}\left(\boldsymbol{e}_{h, k}^{\dot{\boldsymbol{d}}}-\boldsymbol{e}_{h, k-1}^{\dot{\boldsymbol{d}}}\right)\right)
$$

in $(45)_{3}$. Note that we do have $\left(\boldsymbol{v}_{h}^{\mathrm{f}}, \boldsymbol{v}_{h}^{\mathrm{s}}\right) \in \boldsymbol{W}_{h}$, thanks to $(45)_{1}$. We then get

$$
\begin{aligned}
\rho^{\mathrm{f}}\left\|\boldsymbol{e}_{h, k}^{\boldsymbol{u}}\right\|_{0, \Omega^{\mathrm{f}}}^{2}+ & \mu \tau\left\|\boldsymbol{e}_{h, k}^{\boldsymbol{u}}\right\|_{1, \Omega^{\mathrm{f}}}^{2}+\rho^{\mathrm{s}}\left\|\boldsymbol{e}_{h, k}^{\dot{\boldsymbol{d}}}\right\|_{\mathrm{s}, h}^{2}+\left\|\boldsymbol{e}_{h, k}^{\boldsymbol{d}}\right\|_{\mathrm{e}, h}^{2} \\
+ & \tau\left\|\boldsymbol{e}_{h, k}^{\dot{\boldsymbol{d}}}\right\|_{\mathrm{v}, h}^{2}+\underbrace{\tau\left(\boldsymbol{e}_{h, k}^{\boldsymbol{d}}, \boldsymbol{L}_{h}^{\mathrm{e}}\left(\boldsymbol{e}_{h, k}^{\boldsymbol{d}}-\boldsymbol{e}_{h, k-1}^{\boldsymbol{d}}\right)+\boldsymbol{L}_{h}^{\mathrm{v}}\left(\boldsymbol{e}_{h, k}^{\dot{\boldsymbol{d}}}-\boldsymbol{e}_{h, k-1}^{\dot{\boldsymbol{d}}}\right)\right)_{\Omega^{\mathrm{s}}, h}}_{I_{1}} \\
& +\underbrace{\frac{\tau^{2}}{\rho^{\mathrm{s}}}\left(\boldsymbol{L}_{h}^{\mathrm{e}} \boldsymbol{e}_{h, k}^{\boldsymbol{d}}+\boldsymbol{L}_{h}^{\mathrm{v}} \boldsymbol{e}_{h, k}^{\boldsymbol{d}}, \boldsymbol{L}_{h}^{\mathrm{e}}\left(\boldsymbol{e}_{h, k}^{\boldsymbol{d}}-\boldsymbol{e}_{h, k-1}^{\boldsymbol{d}}\right)+\boldsymbol{L}_{h}^{\mathrm{v}}\left(\boldsymbol{e}_{h, k}^{\dot{\boldsymbol{d}}}-\boldsymbol{e}_{h, k-1}^{\dot{\boldsymbol{d}}}\right)\right)_{\Omega^{\mathrm{s}}, h}}_{I_{2}} \leq 0 .
\end{aligned}
$$

The last terms can be controlled following the same argument that in the proof of Theorem 4 with $r=1$. Hence, using $(45)_{2}$, we get

$$
\begin{aligned}
I_{1}= & \frac{1}{2}\left(\left\|\boldsymbol{e}_{k}^{\boldsymbol{d}}\right\|_{\mathrm{e}, h}^{2}-\left\|\boldsymbol{e}_{k-1}^{\boldsymbol{d}}\right\|_{\mathrm{e}, h}^{2}+\left\|\boldsymbol{e}_{k}^{\boldsymbol{d}}-\boldsymbol{e}_{k-1}^{\boldsymbol{d}}\right\|_{\mathrm{e}, h}^{2}\right) \\
& +\frac{\tau}{2}\left(\left\|\boldsymbol{e}_{k}^{\dot{\boldsymbol{d}}}\right\|_{\mathrm{v}, h}^{2}-\left\|\boldsymbol{e}_{k-1}^{\dot{\boldsymbol{d}}}\right\|_{\mathrm{v}, h}^{2}+\left\|\boldsymbol{e}_{k}^{\dot{\boldsymbol{d}}}-\boldsymbol{e}_{k-1}^{\dot{\boldsymbol{d}}}\right\|_{\mathrm{v}, h}^{2}\right) \\
I_{2}= & \frac{\tau^{2}}{2 \rho^{\mathrm{s}}}\left(\left\|\boldsymbol{L}_{h}^{\mathrm{e}} \boldsymbol{e}_{k}^{\boldsymbol{d}}+\boldsymbol{L}_{h}^{\mathrm{v}} \boldsymbol{e}_{k}^{\dot{\boldsymbol{d}}}\right\|_{\mathrm{s}, h}^{2}-\left\|\boldsymbol{L}_{h}^{\mathrm{e}} \boldsymbol{e}_{k-1}^{\boldsymbol{d}}+\boldsymbol{L}_{h}^{\mathrm{v}} \boldsymbol{e}_{k-1}^{\dot{\boldsymbol{d}}}\right\|_{\mathrm{s}, h}^{2}\right. \\
& \left.+\left\|\boldsymbol{L}_{h}^{\mathrm{e}}\left(\boldsymbol{e}_{k}^{\boldsymbol{d}}-\boldsymbol{e}_{k-1}^{\boldsymbol{d}}\right)+\boldsymbol{L}_{h}^{\mathrm{v}}\left(\boldsymbol{e}_{k}^{\dot{\boldsymbol{d}}}-\boldsymbol{e}_{k-1}^{\dot{\boldsymbol{d}}}\right)\right\|_{\mathrm{s}, h}^{2}\right)
\end{aligned}
$$

The estimate (44) then follows by inserting this expressions into (46), summing over $k=1, \ldots, \infty$ and using Remark 5. This concludes the proof. $\diamond$

To the best of our knowledge, Theorem 5 is the first result which guarantees the convergence of a Robin-Neumann iterative procedure towards the implicit coupling solution (23). From the above proof, one can infer that the structure of the interface Robin operator $\left(\rho^{\mathbf{s}}\right) / \tau \boldsymbol{B}_{h}$ is a fundamental ingredient in the convergence of the iterations. Furthermore, for the initialization $\left(\boldsymbol{d}_{h, 0}, \dot{\boldsymbol{d}}_{h, 0}\right)=\left(\boldsymbol{d}_{h}^{n-1}, \dot{\boldsymbol{d}}_{h}^{n-1}\right)$, the estimate (44) shows that reducing the time-step length $\tau$ increases the convergence speed of the iterations. This will be illustrated in Section 6.1.2 through numerical experiments. 


\section{The non-linear case}

In this section, we formulate the generalized Robin-Neumann schemes of Section 3.3 within a fully non-linear framework, involving a viscous incompressible fluid and a thick-walled non-linear structure. The fluid is described by the incompressible Navier-Stokes equations in ALE formalism and the structure by the non-linear (visco-)elastodynamics equations.

\subsection{The non-linear coupled problem}

Let $\Omega=\Omega^{\mathrm{f}} \cup \Omega^{\mathrm{s}}$ be a reference configuration of the system. The current configuration of the fluid domain, $\Omega^{\mathrm{f}}(t)$, is parametrized by the ALE map $\mathcal{A} \stackrel{\text { def }}{=} \boldsymbol{I}_{\Omega_{\mathrm{f}}}+\boldsymbol{d}_{\mathrm{f}}$ as $\Omega^{\mathrm{f}}(t)=\mathcal{A}\left(\Omega^{\mathrm{f}}, t\right)$, where $\boldsymbol{d}^{\mathrm{f}}: \Omega^{\mathrm{f}} \times \mathbb{R}^{+} \rightarrow \mathbb{R}^{d}$ stands for the displacement of the fluid domain. In practice, $\boldsymbol{d}^{\mathrm{f}}=\operatorname{Ext}\left(\left.\boldsymbol{d}\right|_{\Sigma}\right)$, where $\operatorname{Ext}(\cdot)$ denotes any reasonable lifting operator from the (reference) interface $\Sigma$ into the (reference) fluid domain $\Omega^{\mathrm{f}}$. The strong form of the non-linear fluid-structure problem reads as follows: find the fluid velocity $\boldsymbol{u}: \Omega^{\mathrm{f}} \times \mathbb{R}^{+} \rightarrow \mathbb{R}^{d}$, the fluid pressure $p: \Omega^{\mathrm{f}} \times \mathbb{R}^{+} \rightarrow \mathbb{R}$, the structure displacement $\boldsymbol{d}: \Omega^{\mathrm{s}} \times \mathbb{R}^{+} \rightarrow \mathbb{R}^{d}$ and the structure velocity $\dot{\boldsymbol{d}}: \Omega^{\mathrm{s}} \times \mathbb{R}^{+} \rightarrow \mathbb{R}^{d}$ such that

$$
\begin{aligned}
& \left\{\begin{aligned}
\left.\rho^{\mathrm{f}} \partial_{t}\right|_{\mathcal{A}} \boldsymbol{u}+\rho^{\mathrm{f}}(\boldsymbol{u}-\boldsymbol{w}) \cdot \nabla \boldsymbol{u}-\operatorname{div} \boldsymbol{\sigma}^{\mathrm{f}}(\boldsymbol{u}, p)=\mathbf{0} & \text { in } \quad \Omega^{\mathrm{f}}(t), \\
\operatorname{div} \boldsymbol{u}=0 & \text { in } \quad \Omega^{\mathrm{f}}(t), \\
\boldsymbol{\sigma}^{\mathrm{f}}(\boldsymbol{u}, p) \boldsymbol{n}^{\mathrm{f}}=\boldsymbol{f}^{\Gamma} & \text { on } \quad \Gamma,
\end{aligned}\right. \\
& \begin{array}{r}
\left\{\begin{array}{rlc}
\rho^{\mathrm{s}} \partial_{t} \dot{\boldsymbol{d}}+\alpha \rho^{\mathrm{s}} \dot{\boldsymbol{d}}-\operatorname{div} \boldsymbol{\Pi}(\boldsymbol{d}, \dot{\boldsymbol{d}})=\mathbf{0} & \text { in } & \Omega^{\mathrm{s}}, \\
\dot{\boldsymbol{d}}=\partial_{t} \boldsymbol{d} & \text { in } & \Omega^{\mathrm{s}}, \\
\boldsymbol{d}=\mathbf{0}, \beta \dot{\boldsymbol{d}}=\mathbf{0} & \text { on } & \Gamma^{\mathrm{d}}, \\
\boldsymbol{\Pi}(\boldsymbol{d}, \dot{\boldsymbol{d}}) \boldsymbol{n}^{\mathrm{s}}=\mathbf{0} & \text { on } & \Gamma^{\mathrm{n}},
\end{array}\right. \\
\left\{\begin{array}{rll}
\boldsymbol{d}^{\mathrm{f}}=\operatorname{Ext}\left(\left.\mathrm{d}\right|_{\Sigma}\right), \quad \mathrm{w}=\partial_{\mathrm{t}} \mathrm{d}^{\mathrm{f}} & \text { on } & \Omega^{\mathrm{f}}, \\
\boldsymbol{u}=\boldsymbol{w} & \text { on } & \Sigma(t), \\
\boldsymbol{\Pi}(\boldsymbol{d}, \dot{\boldsymbol{d}}) \boldsymbol{n}^{\mathrm{s}}=-J \boldsymbol{\sigma}^{\mathrm{f}}(\boldsymbol{u}, p) \boldsymbol{F}^{-\mathrm{T}} \boldsymbol{n}^{\mathrm{f}} & \text { on } & \Sigma,
\end{array}\right.
\end{array}
\end{aligned}
$$

where $\left.\partial_{t}\right|_{\mathcal{A}}$ represents the ALE time derivative, $\boldsymbol{F} \stackrel{\text { def }}{=} \boldsymbol{\nabla} \mathcal{A}$ the fluid domain gradient of deformation and $J \stackrel{\text { def }}{=} \operatorname{det} \boldsymbol{F}$ the Jacobian. As usual, a field defined in the reference fluid domain, $\Omega^{\mathrm{f}}$, is evaluated in the current fluid domain, $\Omega^{\mathrm{f}}(t)$, by composition with $\mathcal{A}^{-1}(\cdot, t)$.

In (48), the stress tensor $\Pi(\boldsymbol{d}, \dot{\boldsymbol{d}})$ is defined by the relation $\boldsymbol{\Pi}(\boldsymbol{d}, \dot{\boldsymbol{d}}) \stackrel{\text { def }}{=} \boldsymbol{\pi}(\boldsymbol{d})+\beta \boldsymbol{\pi}^{\prime}(\boldsymbol{d}) \dot{\boldsymbol{d}}$, where $\boldsymbol{\pi}(\boldsymbol{d})$ denotes the first Piola-Kirchhoff tensor of the structure (related to the displacement $\boldsymbol{d}$ through an appropriate constitutive law) and $\boldsymbol{\pi}^{\prime}(\boldsymbol{d})$ stands for Fréchet derivative of $\boldsymbol{\pi}$ at $\boldsymbol{d}$. Physical damping in the solid is hence described through the Rayleigh-like term $\alpha \rho^{\mathrm{s}} \dot{\boldsymbol{d}}-$ $\operatorname{div}\left(\beta \boldsymbol{\pi}^{\prime}(\boldsymbol{d}) \dot{\boldsymbol{d}}\right)$ in $(48)_{1}$, with $\alpha, \beta \geq 0$.

\subsection{Explicit coupling schemes}

The proposed fully explicit coupling schemes combine the explicit treatment of the interface geometrical compatibility $(49)_{1}$ with the following Robin-Neumann time-stepping of the interface kinematical/kinetic coupling $(49)_{2,3}$ on $\Sigma$ :

$$
\left\{\begin{aligned}
J^{n} \boldsymbol{\sigma}^{\mathrm{f}}\left(\boldsymbol{u}^{n}, p^{n}\right)\left(\boldsymbol{F}^{n}\right)^{-\mathrm{T}} \boldsymbol{n}^{\mathrm{f}}+\frac{\rho^{\mathrm{s}}}{\tau} \boldsymbol{B}_{h} \boldsymbol{u}^{n} & =\frac{\rho^{\mathrm{s}}}{\tau} \boldsymbol{B}_{h}\left(\dot{\boldsymbol{d}}^{n-1}+\tau \partial_{\tau} \dot{\boldsymbol{d}}^{\star}\right)-\boldsymbol{\Pi}^{\star} \boldsymbol{n}^{\mathrm{s}}, \\
\boldsymbol{\Pi}^{n} \boldsymbol{n}^{\mathrm{s}} & =-J^{n} \boldsymbol{\sigma}^{\mathrm{f}}\left(\boldsymbol{u}^{n}, p^{n}\right)\left(\boldsymbol{F}^{n}\right)^{-\mathrm{T}} \boldsymbol{n}^{\mathrm{f}},
\end{aligned}\right.
$$

$\mathrm{RR} \mathrm{n}^{\circ} 8384$ 
Algorithm 3 Generalized Robin-Neumann schemes (non-linear version).

For $n \geq r+1$ :

1. Fluid domain update:

$$
\boldsymbol{d}^{\mathrm{f}, n}=\operatorname{Ext}\left(\left.\boldsymbol{d}^{n-1}\right|_{\Sigma}\right), \quad \boldsymbol{w}^{n}=\partial_{\tau} \boldsymbol{d}^{\mathrm{f}, n}, \quad \mathcal{A}^{n} \stackrel{\text { def }}{=} \boldsymbol{I}_{\Omega}+\boldsymbol{d}^{\mathrm{f}, n}, \quad \Omega^{n} \stackrel{\text { def }}{=} \mathcal{A}^{n}(\Omega)
$$

and we set $\boldsymbol{F}^{n}=\boldsymbol{\nabla} \mathcal{A}^{n}$ and $J^{n}=\operatorname{det} \boldsymbol{F}^{n}$.

2. Fluid step: find $\boldsymbol{u}^{n}: \Omega \times \mathbb{R}^{+} \rightarrow \mathbb{R}^{d}$ and $p^{n}: \Omega \times \mathbb{R}^{+} \rightarrow \mathbb{R}$ such that

$$
\left\{\begin{array}{rccc}
\left.\rho^{\mathrm{f}} \partial_{\tau}\right|_{\mathcal{A}} \boldsymbol{u}^{n}+\rho^{\mathrm{f}}\left(\boldsymbol{u}^{n-1}-\boldsymbol{w}^{n}\right) \cdot \boldsymbol{\nabla} \boldsymbol{u}^{n}-\operatorname{div} \boldsymbol{\sigma}^{\mathrm{f}}\left(\boldsymbol{u}^{n}, p^{n}\right)=\mathbf{0} & \text { in } & \Omega^{\mathrm{f}, n} \\
\operatorname{div} \boldsymbol{u}^{n}=0 & \text { in } & \Omega^{\mathrm{f}, n} \\
\boldsymbol{\sigma}^{\mathrm{f}}\left(\boldsymbol{u}^{n}, p^{n}\right) \boldsymbol{n}^{\mathrm{f}}=\boldsymbol{f}^{\Gamma}\left(t_{n}\right) & \text { on } & \Gamma, \\
J^{n} \boldsymbol{\sigma}^{\mathrm{f}}\left(\boldsymbol{u}^{n}, p^{n}\right)\left(\boldsymbol{F}^{n}\right)^{-\mathrm{T}} \boldsymbol{n}^{\mathrm{f}}+\frac{\rho^{\mathrm{s}}}{\tau} \boldsymbol{B}_{h} \boldsymbol{u}^{n}=\frac{\rho^{\mathrm{s}}}{\tau} \boldsymbol{B}_{h}\left(\dot{\boldsymbol{d}}^{n-1}+\tau \partial_{\tau} \dot{\boldsymbol{d}}^{\star}\right) & & \\
-\boldsymbol{\Pi}^{\star} \boldsymbol{n}^{\mathrm{s}} & \text { on } \quad \Sigma .
\end{array}\right.
$$

3. Solid step: find $\boldsymbol{d}^{n}: \Omega^{\mathrm{s}} \times \mathbb{R}^{+} \rightarrow \mathbb{R}^{d}$ and $\dot{\boldsymbol{d}}^{n}: \Omega^{\mathrm{s}} \times \mathbb{R}^{+} \rightarrow \mathbb{R}^{d}$ such that

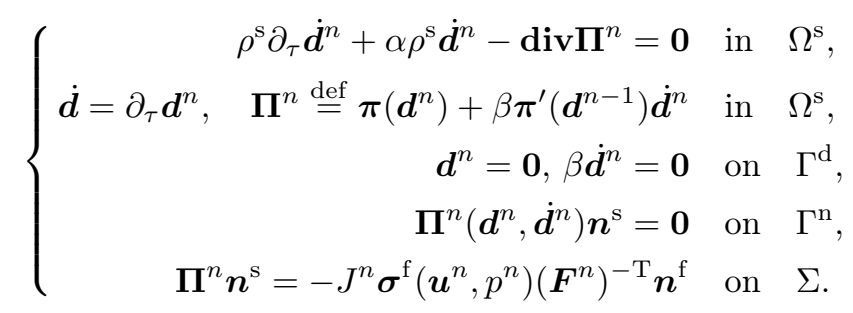

derived from the arguments introduced in Section 3.3. The solid stress tensor is given by the expression $\boldsymbol{\Pi}^{n} \stackrel{\text { def }}{=} \boldsymbol{\pi}\left(\boldsymbol{d}^{n}\right)+\beta \boldsymbol{\pi}^{\prime}\left(\boldsymbol{d}^{n-1}\right) \dot{\boldsymbol{d}}^{n}$, which involves a semi-implicit treatment of the viscous contribution.

The resulting time-marching procedures are detailed in Algorithm 3.

\section{$6 \quad$ Numerical experiments}

In this section, we investigate through numerical experiments the properties of the explicit coupling scheme introduce above. Several fluid-structure interaction examples from the literature have been considered. Section 6.1 presents a convergence study in 2D, using the linear model problem (1)-(3). Numerical results based on the non-linear model (49)-(49), with a Saint VenantKirchhoff constitutive law for the solid and 3D geometries, are presented in the subsequent sections.

\subsection{Numerical study in a two-dimensional test-case}

The first example is the popular two-dimensional pressure-wave propagation benchmark (see, e.g., [1]). We consider (1)-(3) with $\Omega^{\mathrm{f}}=[0, L] \times[0, R], \Omega^{\mathrm{s}}=[0, L] \times[R, R+\epsilon], L=6, R=0.5$ 
and $\epsilon=0.1$. All the units are given in the CGS sytem. At the fluid boundary $x=0$ we impose a sinusoidal pressure of maximal amplitude $2 \times 10^{4}$ during $5 \times 10^{-3}$ time instants, corresponding to half a period. Zero traction is enforced at $x=6$ and a symmetry condition is applied on the lower wall. The solid is clamped at its extremities and zero traction is enforced on its upper boundary. The fluid physical parameters are given by $\rho^{\mathrm{f}}=1$ and $\mu=0.035$. For the solid we have $\rho^{\mathrm{s}}=1.1, L_{1}=1.15 \cdot 10^{6}, L_{2}=1.7 \cdot 10^{6}, c_{0}=4 \cdot 10^{6}, \alpha \rho^{\mathrm{s}}=10^{-3}, \beta=10^{-3}$. All the computations have been performed with FreeFem ++ (see [19]).

For illustration purposes, we have reported in Figure 2 a few snapshots of the pressure field obtained using Algorithm 1 with $r=1, \tau=10^{-4}$ and $h=0.05$. The fluid and solid domains have been displayed in deformed configuration (amplified by a factor 10). The numerical solution remains stable, as predicted by Theorem 4, and a propagating pressure-wave is observed.

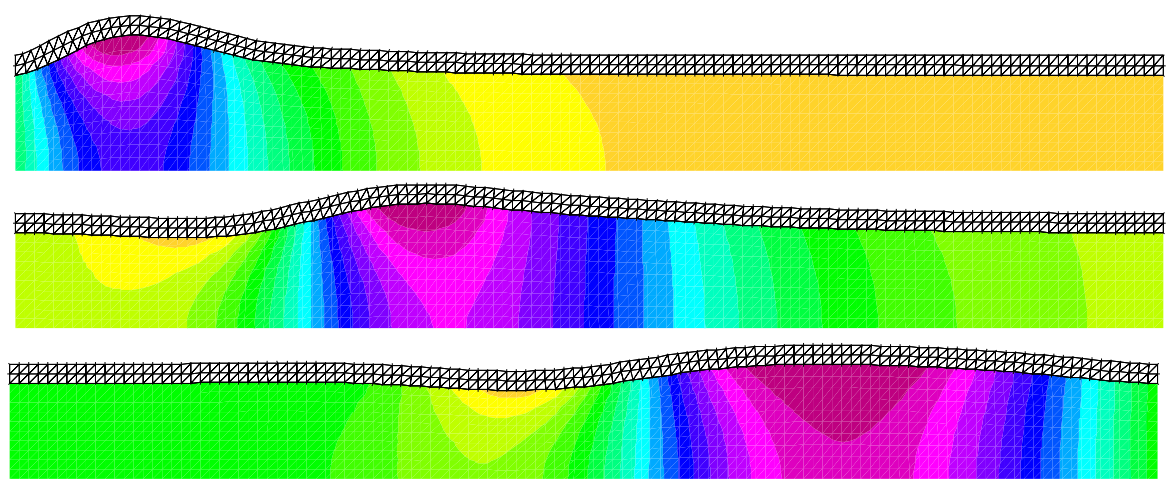

Figure 2: Snapshots of the fluid pressure and solid deformation at $t=4 \cdot 10^{-3}, 9 \cdot 10^{-3}$ and $15 \cdot 10^{-3}$ (from top to bottom). Algorithm 1 with $r=1, \tau=10^{-4}$ and $h=0.05$.

\subsubsection{Accuracy of the explicit coupled schemes}

We first compare the interface vertical displacement obtained with Algorithm $1(r=0$ and $r=1)$ and the implicit scheme for $h=0.05$ and $\tau=10^{-4}, 5 \cdot 10^{-5}, 2 \cdot 10^{-5}$. A reference solution has been generated using the implicit scheme and a high grid resolution $\left(\tau=10^{-6}, h=3.125 \cdot 10^{-3}\right)$. The corresponding results are reported in Figure 3 . We can clearly observe that the extrapolation order $r$ has a major impact on the accuracy of Algorithm 1, as suggested by (30). The choice $r=0$ yields a very poor accuracy, while for $r=1$ seems to be only slightly less accurate than the implicit scheme. Accordingly with (30), as the time step $\tau$ tends to zero, the numerical solution obtained with $r=1$ reaches the implicit coupling solution.

The results of Algorithm 1 with $r=2$ are not reported in Figure 3 since the scheme is unstable for the set of physical and discretization parameters considered. The stability condition (37) is very restrictive in this case (parabolic-CFL condition). In order to provide a global overview of all the variants (including the case $r=2$ ) we propose to switch off the solid damping (i.e., $\alpha=\beta=0$ ). According to Theorem 4 this yields a weakened $6 / 5$-CFL stability condition. The results obtained with $\tau=2 \cdot 10^{-5}$ and $h=0.05$ are reported in Figure 4. Once more, for $r=0$ we get a very poor approximation. Algorithm 1 with $r=1$ and $r=2$ yields practically the same solution than the implicit scheme.

We now investigate the impact of the spatial discretization on the size of the kinematic perturbation (30). For this purpose, we present in Figure 5 the results obtained with $\tau=10^{-4}$ and $h=0.025$. By comparing with Figure 3(a) we see that, for a fixed time-step length, the 


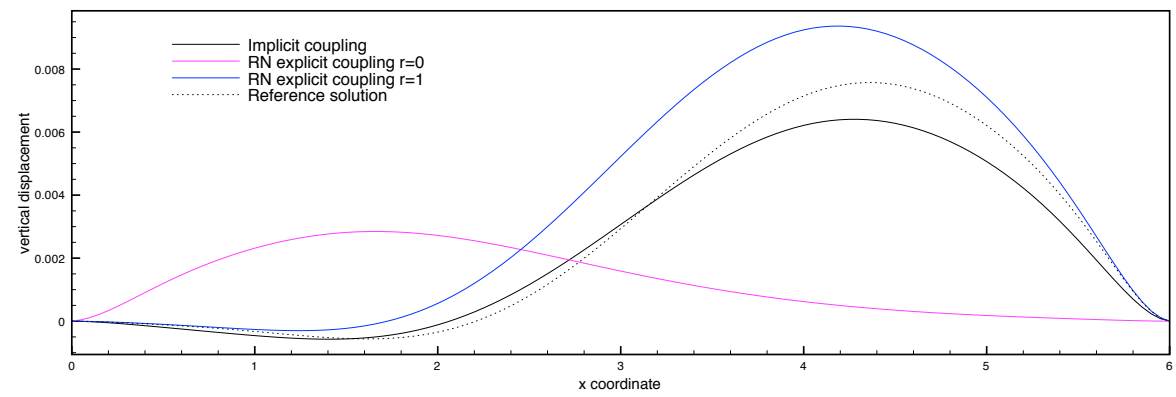

(a) $\tau=10^{-4}$.

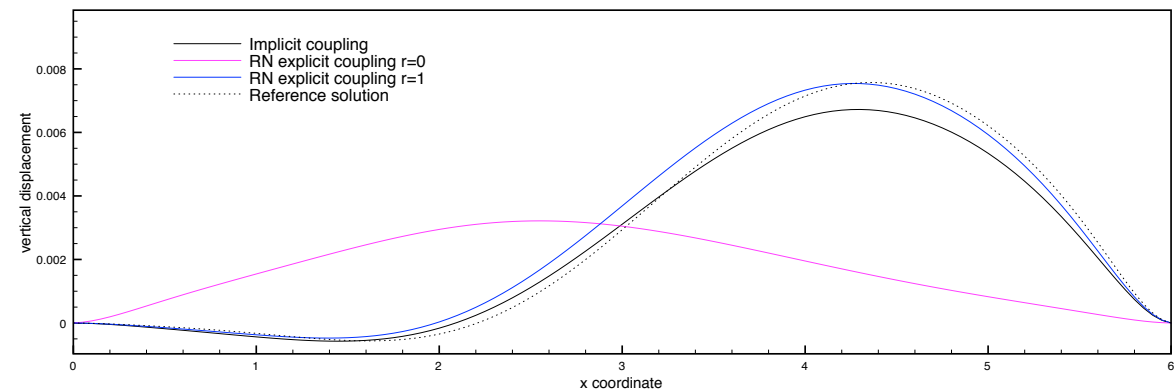

(b) $\tau=5 \cdot 10^{-5}$.

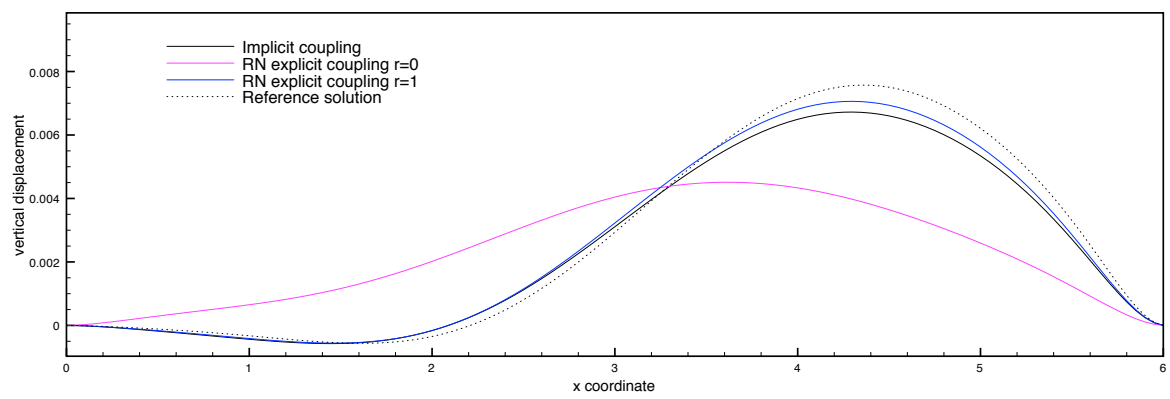

(c) $\tau=2 \cdot 10^{-5}$

Figure 3: Interface vertical displacement at time $t=1.5 \cdot 10^{-2}$, with $h=0.05$ (damped solid, $\left.\beta=10^{-3}\right)$.

accuracy of Algorithm 1 deteriorates under spatial refinement. This behavior is even more striking in Figure 6 where we report the vertical displacement obtained for several values of $h$ (and $\tau=10^{-4}$ fixed). This clearly indicates a non-uniformity in $h$ of the truncation error induced by the kinematic perturbation (30).

In order to provide a complete insight on the accuracy of the schemes, Figure 7(a) presents the convergence histories of the solid displacement relative energy error at time $t=1.5 \cdot 10^{-2}$ obtained with Algorithm 1 and the implicit coupling scheme, by refining both in space and in time under a hyperbolic-CFL constraint $(\tau=h / 200)$. The variant with $r=0$ is unable to show a convergent behavior towards the reference solution. On the contrary, the scheme with $r=1$ shows a convergence rate between $1 / 2$ and 1 . The superior accuracy of the the implicit scheme is clearly visible, for which we recover the expected first-order optimal rate. Note that Algorithm 1 


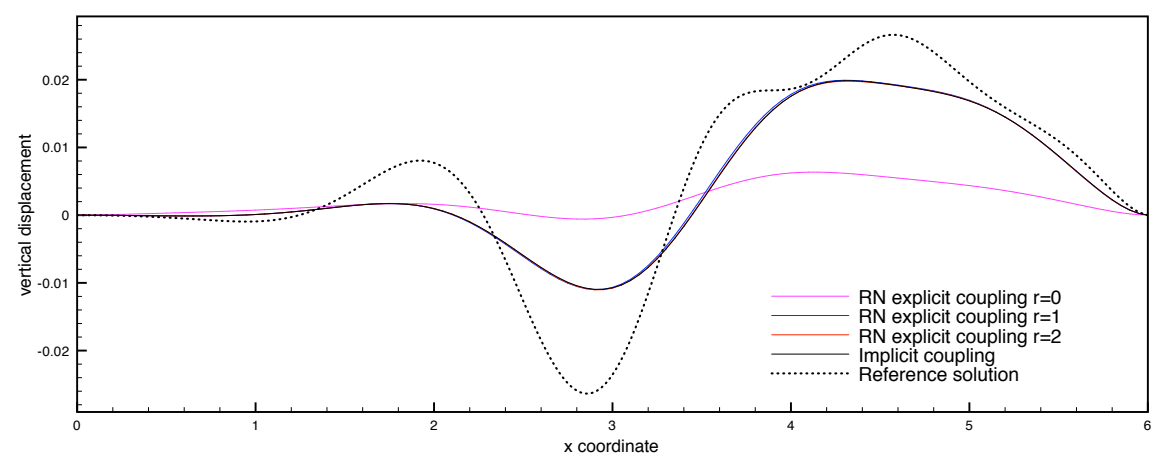

Figure 4: Interface vertical displacement at time $t=1.5 \cdot 10^{-2}$, with $\tau=2 \cdot 10^{-5}$ and $h=0.05$, (undamped solid, $\beta=0$ ).

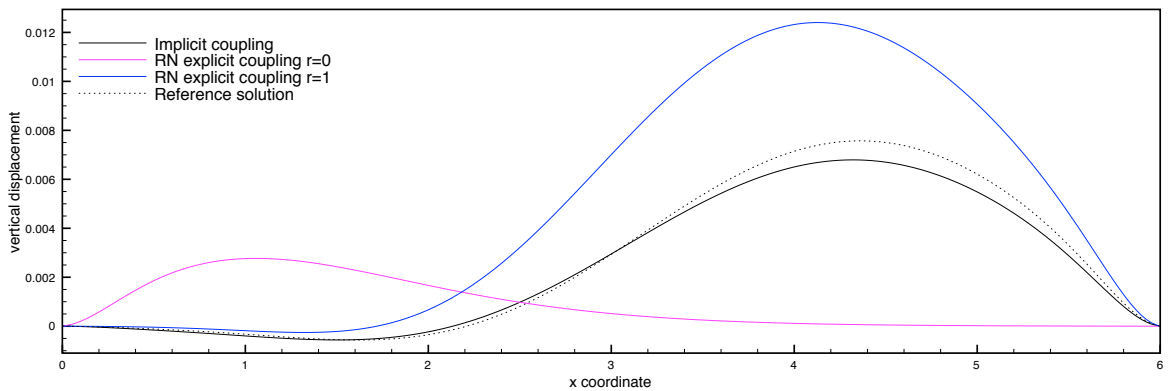

Figure 5: Interface vertical displacement at time $t=1.5 \cdot 10^{-2}$ for $\tau=10^{-4}$ and $h=0.01$.

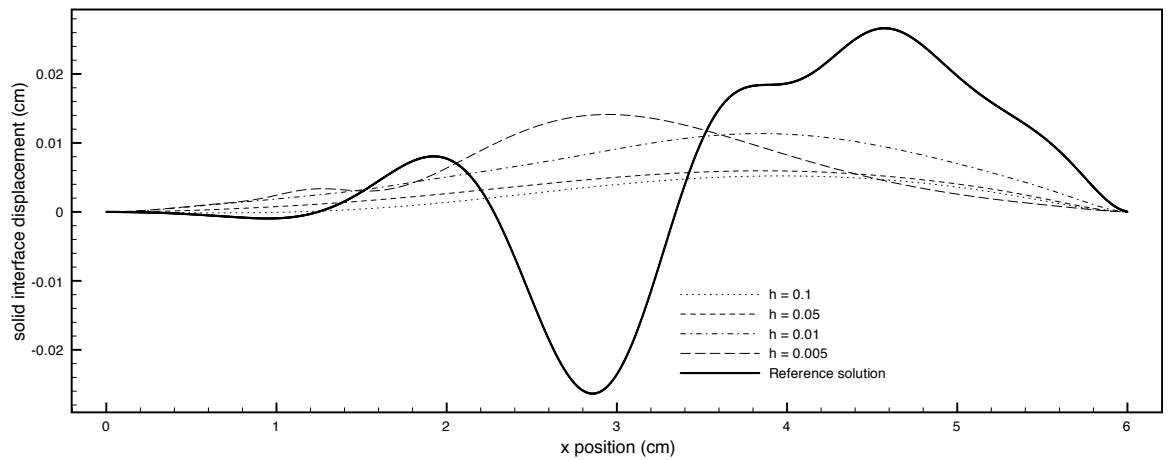

Figure 6: Interface vertical displacement at time $t=1.5 \cdot 10^{-2}$. Algorithm 1 with $r=1$ and $\tau=10^{-4}$.

with $r=1$ yields convergence under the standard hyperbolic-CFL constraint (without the need of corrections iterations). This is a significant progress with respect to the stabilized explicit coupling scheme reported in [4], for which convergence demands strengthened CFL conditions $($ see $[9,5])$.

In Figure 7(b), we report the convergence histories obtained under a parabolic-CFL constraint $\left(\tau=h^{2} / 100\right)$ and without damping in the solid $(\alpha=\beta=0)$. In this case, the explicit variant 


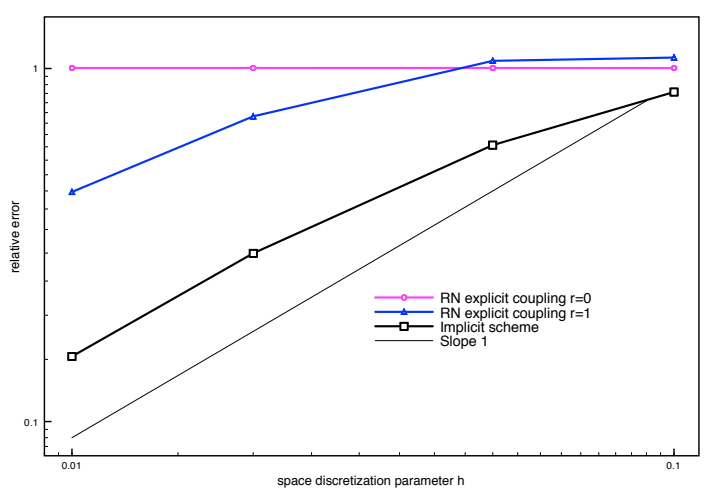

(a) $\tau=\mathcal{O}(h)$ and damped solid.

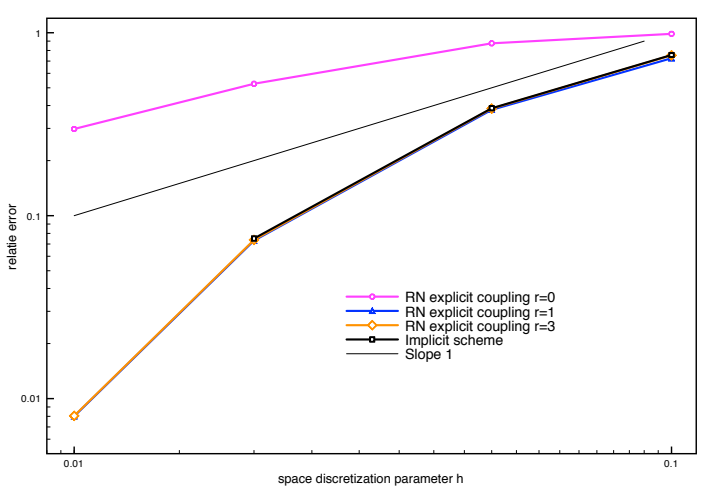

(b) $\tau=\mathcal{O}\left(h^{2}\right)$ and undamped solid.

Figure 7: Convergence history of the solid displacement relative energy error at time $t=1.5 \cdot 10^{-2}$.

with $r=0$ shows a convergent behavior, with a rate between $1 / 2$ and 1 . A superior convergent behavior is observed for the explicit schemes with $r=1$ and $r=2$ and the implicit scheme, which yield practically the same rate.

In view of the results reported in Figure 7, we postulate the following rates for the consistency of the kinematic perturbation (30):

- $r=0: \mathcal{O}\left((\tau / h)^{\frac{1}{2}}\right)$;

- $r=1: \mathcal{O}\left(\tau / h^{\frac{1}{2}}\right)$;

- $r=2: \mathcal{O}\left(\tau^{2} / h^{\frac{1}{2}}\right)$.

The factor $h^{-\frac{1}{2}}$ could be related to a non-uniformity in $h$ of the solid discrete operators $\boldsymbol{L}_{h}^{\mathrm{e}}$ and $\boldsymbol{L}_{h}^{\mathrm{v}}$. It is worth noting that this sub-optimality is not present in the original Robin-Neumann explicit schemes (see $[13,10]$ ), in the case of the coupling with a thin-walled structure.

\subsubsection{Partitioned solution of implicit coupling}

In this section we investigate numerically the convergence properties of the (parameter free) iterative procedure given by Algorithm 2. Figure 8 reports the mean number of iterations per time-step needed to simulate the wave propagation until $t=5 \cdot 10^{-3}$, for different values of $\tau, h, \rho^{\mathrm{s}}$, Young Modulus $E$ and domain length $L$. We compare the performance of Algorithm 2 with the standard Robin-Neumann procedure introduced in [26], using the Robin coefficient $\alpha=\rho^{\mathrm{s}} \epsilon / \tau+c_{0} \tau$ proposed therein. The iterations are initialized from the data of the previous time-step.

Both procedures yield a similar behavior with respect to the solid density and the domain length (see Figures 8(a)-(b)). Figures 8(c)-(d), on the contrary, show that Algorithm 2 is much less sensitive to $\tau$ and $E$ than the standard Robin-Neumann procedure. In fact, as suggested by the error estimate of Theorem 5, reducing $\tau$ enhances the convergence speed of Algorithm 2. This can also be explained in terms of the kinematic relation $(34)_{1}$, since the size of the perturbation is proportional to $\tau E / \rho^{\mathrm{s}}$. Note that the convergence of the standard Robin-Neumann method degrades as $\tau$ goes to zero. This behavior is also highlighted by Figure 9, where we have reported the relative error per iteration for different values of $\tau$. 


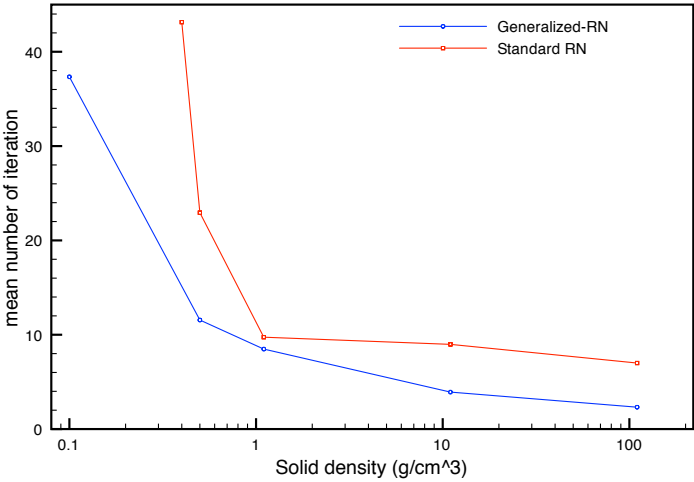

(a) Impact of $\rho^{\mathrm{s}}$.

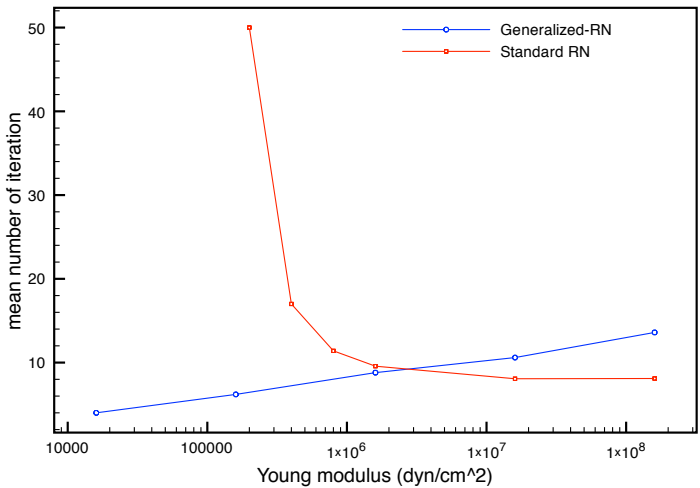

(c) Impact of $E$.

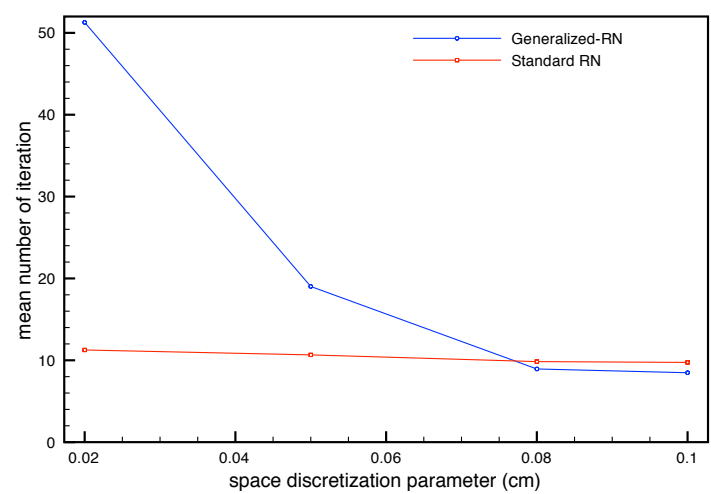

(e) Impact of $h$.

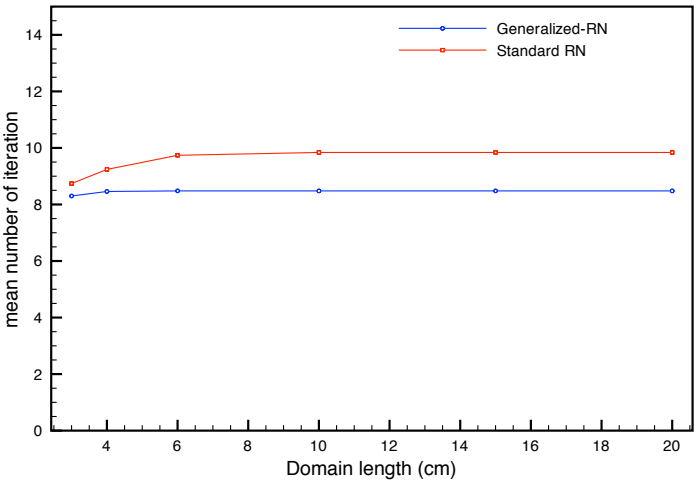

(b) Impact of $L$.

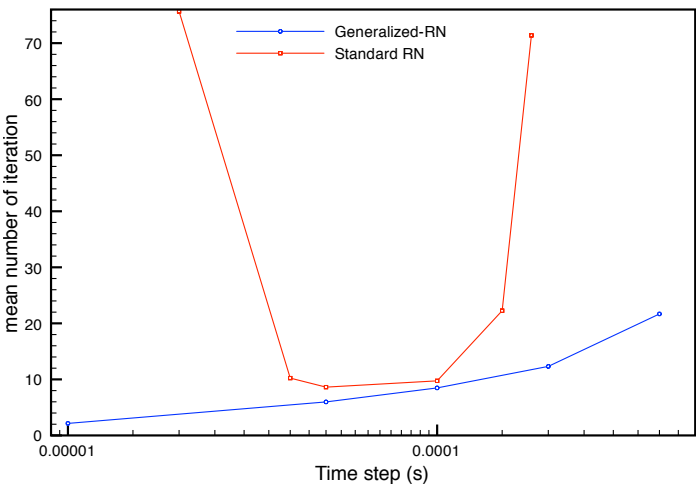

(d) Impact of $\tau$.

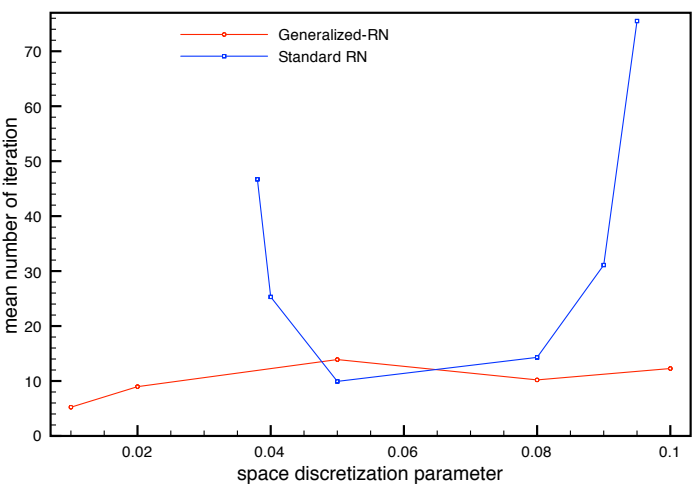

(f) Impact of $h$ and $\tau, \tau=\mathcal{O}\left(h^{2}\right)$.

Figure 8: Sensitivity of the convergence speed of the iterative procedure to the physical and discretization parameters.

In line with the non-uniformity in $h$ observed for the accuracy of Algorithm 1 in Section 6.1.1, the convergence of Algorithm 2 degrades as $h$ goes to zero ( $\tau$ fixed), as shown in Figure 8(e). Finally, Figure 8(f) shows that under a parabolic-CFL condition, the standard Robin-Neumann method losses convergence, whereas the proposed Algorithm 2 keeps a reduced number of itera- 


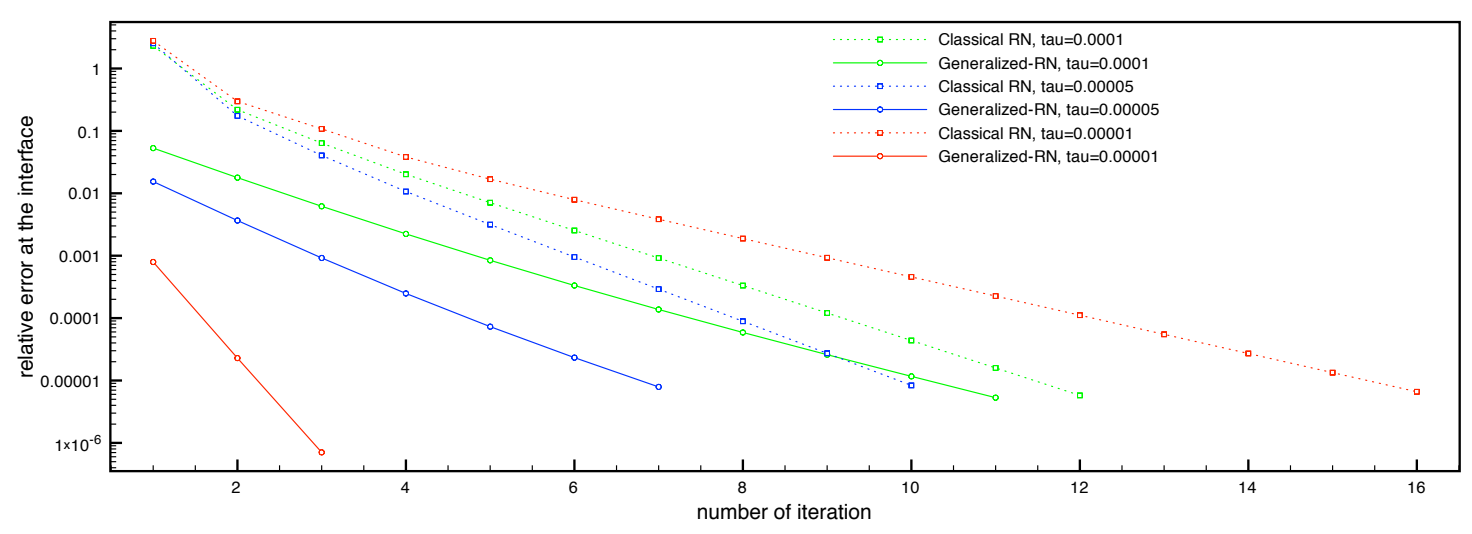

Figure 9: Relative error on the interface against the number of sub-iterations for the RobinNeumann scheme and the corrected scheme with $h=0.1$.

tions.

\subsection{Pressure wave propagation in a straight tube}

We consider the example proposed in in [14] (see also [15, Chapter 12]). The fluid-structure system is modeled by the non-linear coupled problem (47)-(49). The fluid domain is a straight tube of radius 0.5 and length 5 . All the units are given in the CGS system. The vessel wall has a thickness of 0.1 and is clamped at its extremities. The physical parameters for the fluid are $\rho^{\mathrm{f}}=1$ and $\mu=0.035$. For the solid we have $\rho^{\mathrm{s}}=1.2$, Young modulus $E=3 \times 10^{6}$ and Poisson's ratio $\nu=0.3$. The overall system is initially at rest and an over pressure of $1.3332 \times 10^{4}$ is imposed on the inlet boundary during the time interval [0,0.005]. The fluid and solid equations are discretized in space using continuous $\mathbb{P}_{1}$ finite elements (a SUPG/PSPG stabilized formulation is considered in the fluid).
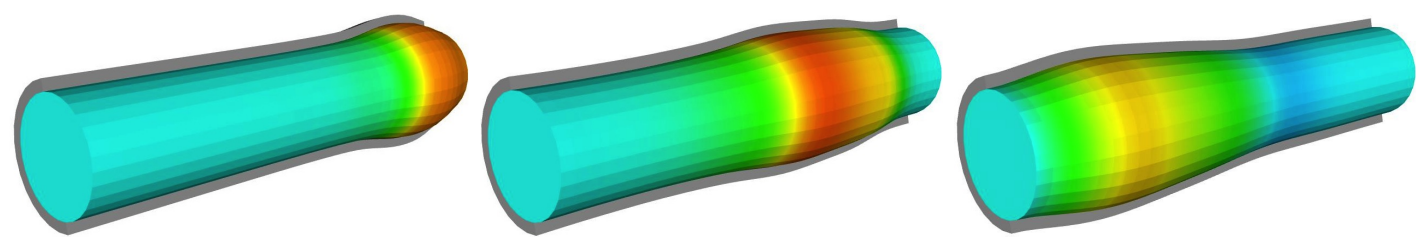

(a) Undamped solid $(\alpha=\beta=0)$.
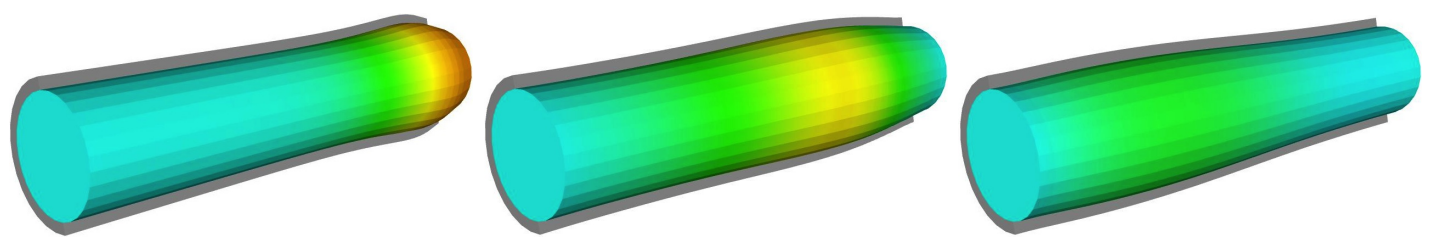

(b) Damped solid $\left(\alpha=1, \beta=10^{-3}\right)$.

Figure 10: Snapshots of the fluid pressure and solid deformation at $t=0.003,0.007,0.012$ (from left to right) obtained with Algorithm $3(r=1)$ and $\tau=10^{-4}$. 
In Figure 10 we have reported some snapshots of the fluid pressure and solid deformation (amplified by a factor 10) obtained with Algorithm $3(r=1)$ and $\tau=10^{-4}$. A stable pressure wave propagation is observed. The impact of the solid damping $\left(\alpha=1, \beta=10^{-3}\right)$ is noticeable.
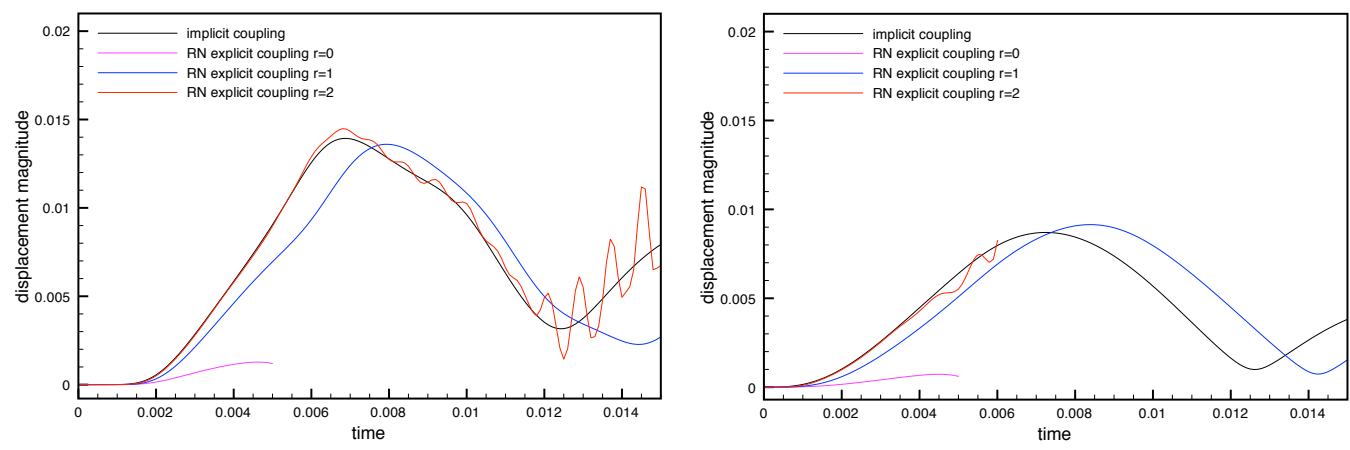

(a) $\tau=10^{-4}$.
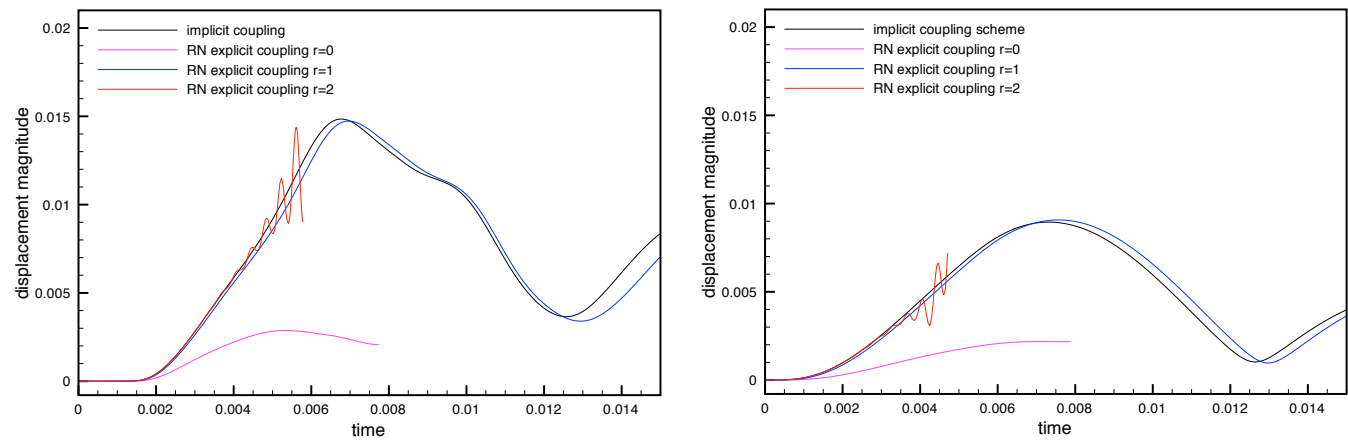

(b) $\tau=4.4721 \cdot 10^{-5}$.
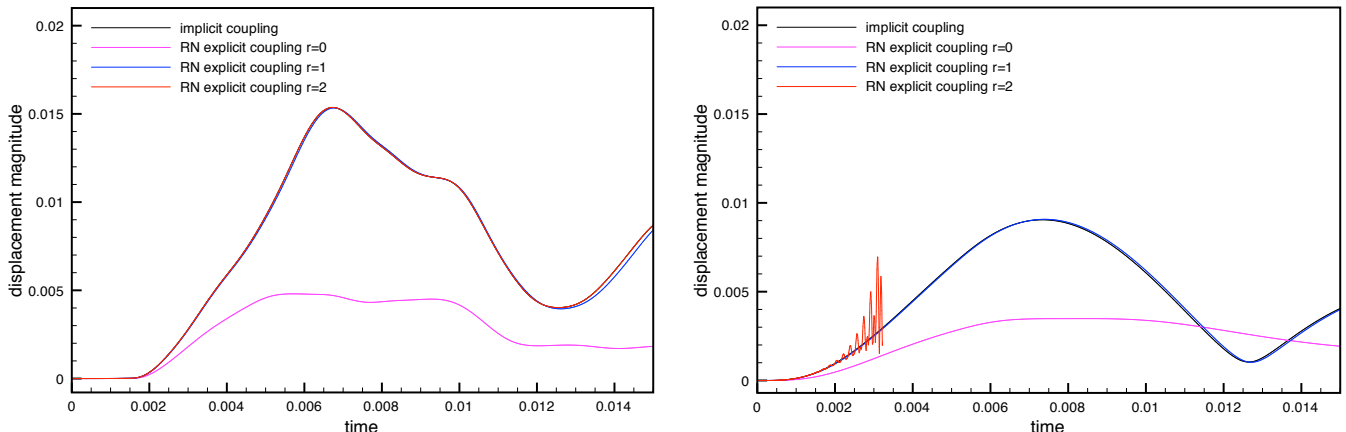

(c) $\tau=2 \cdot 10^{-5}$.

Figure 11: Interface mid-point displacement magnitudes obtained with the implicit coupling scheme and Algorithm 3. Left: undamped solid $(\alpha=\beta=0)$. Right: damped solid $(\alpha=1$, $\left.\beta=10^{-3}\right)$.

For comparison purposes, Figure 11 reports the interface mid-point displacement magnitudes obtained with Algorithm 3 and the implicit coupling scheme. Algorithm 3 with $r=1$ yields an stable numerical solution in all the cases considered, which confirms the unconditional stability stated in Theorem 4. As regards accuracy, the scheme retrieves the overall dynamics of the 
solution provided implicit method, particularly, with the smallest time-step lengths. A phase mismatch is clearly visible with $\tau=10^{-4}$.

Algorithm 3 with $r=0$ yields either a poor approximation or instability at the level of the inlet boundary. The accuracy issue was already observed in Section 6.1.1 with a linear problem. Numerical investigations (not reported here) indicate that the instabilities are the result of an intricate interaction between the low-order perturbation of the kinematic constraint, the nonlinearity of the fluid equation and the natural character of the inlet boundary conditions. This explains the discrepancy with the stability result of Theorem 4 (linear case) and the fact that the spurious oscillations are not visible in Figure 11 (interface mid-point displacement).

Figure 11 points out the restrictive time-step restrictions required by Algorithm 3 with $r=2$. A stable numerical approximation is observed only in the case without solid damping and for the smallest time-step length. This confirms the hybrid hyperbolic/parabolic characteristics of the stability condition (37) in Theorem 4. Though unstable, the high-order perturbation of the kinematic constraint introduced by the explicit scheme with $r=2$ is clearly visible in Figure 11(a).

\subsection{Flow around an elastic object}

In this example we consider the two-dimensional benchmark proposed in [31], describing the flow of a fluid around a cylinder with an attached elastic structure. The fluid-structure system
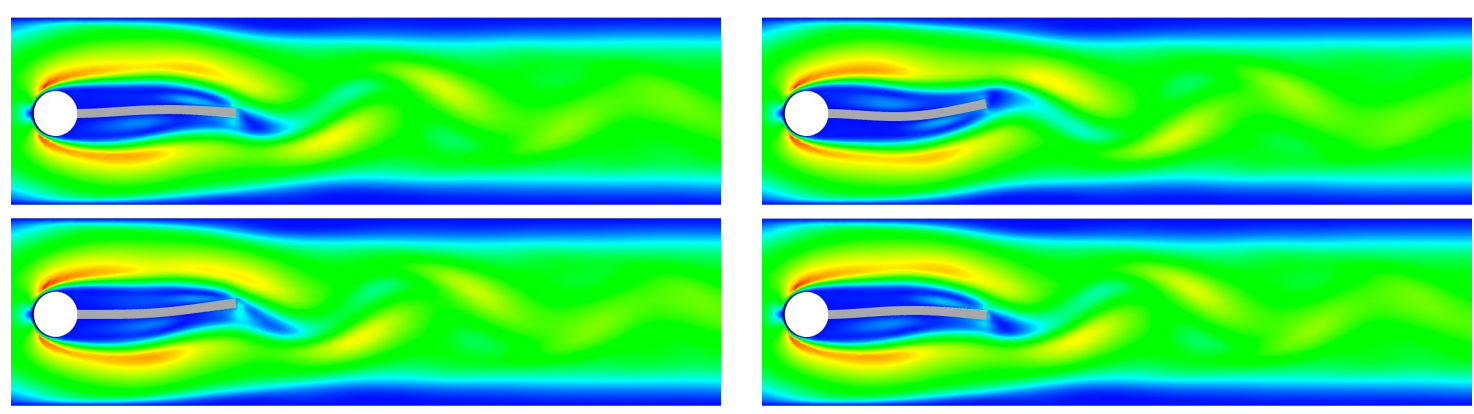

Figure 12: Fluid velocity magnitude and solid deformation at $t=9.65,9.722,9.848$ and 10 (from left to right and top to bottom) obtained with Algorithm $3(r=1)$.

is modeled by the non-linear coupled problem (47)-(49). The reader is referred to [31] for the complete description of the geometry. The fluid and solid are supposed to be initially at rest. A parabolic velocity profile is imposed on the inlet boundary. The mean inflow velocity is denoted by $\bar{U}$. The physical parameters for the fluid are $\rho^{\mathrm{f}}=10^{3}, \mu=10^{-3}$ and $\bar{U}=2$ (i.e., $\operatorname{Re}=200$ ), while for the solid we have $\rho^{\mathrm{s}}=10^{3}, E=5.6 \cdot 10^{6}, \nu=0.4$ and $\alpha=\beta=0$ (undamped solid). All the units are given in the SI system. Among the test-cases proposed in [31], this physical setting (termed FSI3 in [31]) is the most difficult from the point of view of the added-mass effect issues $\left(\rho^{\mathrm{s}}=\rho^{\mathrm{f}}\right)$.

The simulations are performed in three-dimensions, by imposing symmetry conditions along the extrusion direction. The fluid and solid equations are discretized in space using continuous $\mathbb{P}_{1}$ finite elements (a SUPG/PSPG stabilized formulation is considered in the fluid). The timestep length is $\tau=10^{-3}$ and a total number of 86912 and 1830 degrees-of-freedom is considered, respectively, for the fluid and the solid. This is among the coarsest space-time resolutions considered in [31]. For illustration purposes, some snapshots of the fluid velocity magnitude and solid 


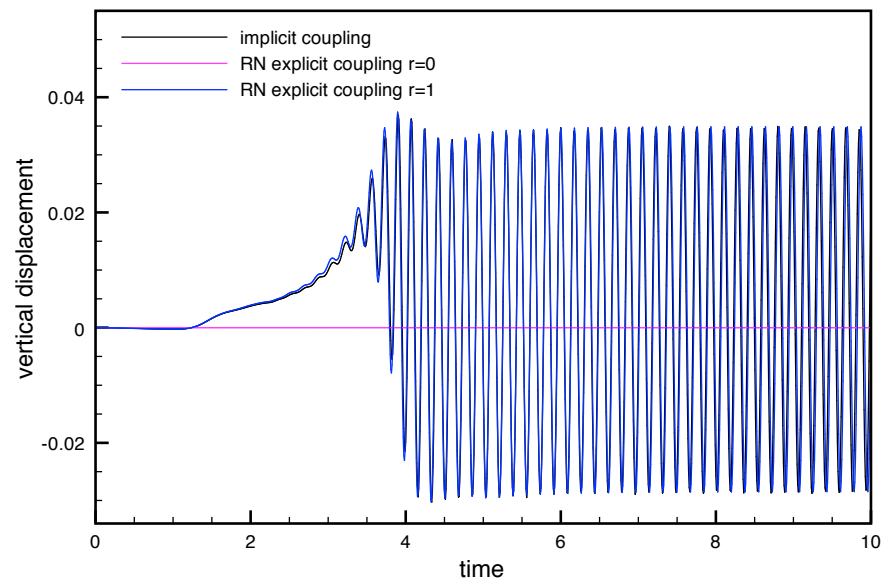

Figure 13: Vertical displacement at an interface node obtained with the implicit coupling scheme and Algorithm $3\left(\tau=10^{-3}\right)$.

deformation obtained with Algorithm $3(r=1)$ are reported in Figure 12. An stable solution involving periodic self-excited oscillations of large amplitude is observed.

Figure 13 reports the interface mid-point displacement magnitudes obtained with Algorithm 3 ( $r=0$ and $r=1)$ and the implicit coupling scheme. The poor accuracy of the explicit coupling scheme with $r=0$ is striking. On the contrary, the solution obtained with $r=1$ is practically indistinguishable from the one provided by the implicit coupling scheme. This enhanced accuracy, with respect to the results reported in Section 6.2, can be explained by the fact that increasing the solid density reduces the impact of the kinematic perturbation in (30). Numerical investigations (not reported here) showed that, for this set of discretization parameters, Algorithm 3 with $r=2$ is unstable and that smaller time-steps are needed for stability.

\subsection{Damped structural instability}

We consider an adaptation of the balloon-type fluid-structure example proposed in [23]. A curved

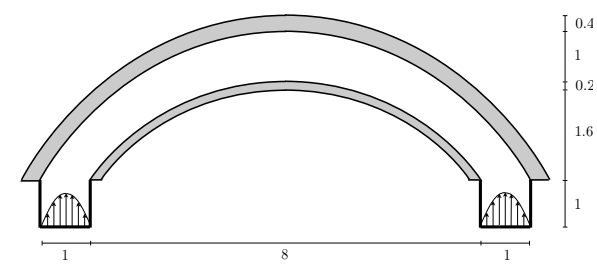

Figure 14: A bended fluid domain surrounded by two structures.

fluid domain is surrounded by two structures with different stiffness (see Figure 14). Both structures are fixed on their extremities. A parabolic velocity profile is prescribed on the left and right inflow boundaries, with maximal magnitudes 10 and 10.2, respectively (to avoid perfect symmetry). All the units are given in the SI system. Zero velocity is enforced on the remaining fluid boundaries. The fluid-structure system is modeled by the coupled problem (47)-(49). The fluid is loaded with the volume force $\boldsymbol{f}=(0,-1)^{\mathrm{T}}$. The fluid physical parameters are given by $\rho^{\mathrm{f}}=1.0$ and $\mu=9$, while for the top and bottom (undamped) structures we have $\rho^{\mathrm{s}}=500$, 

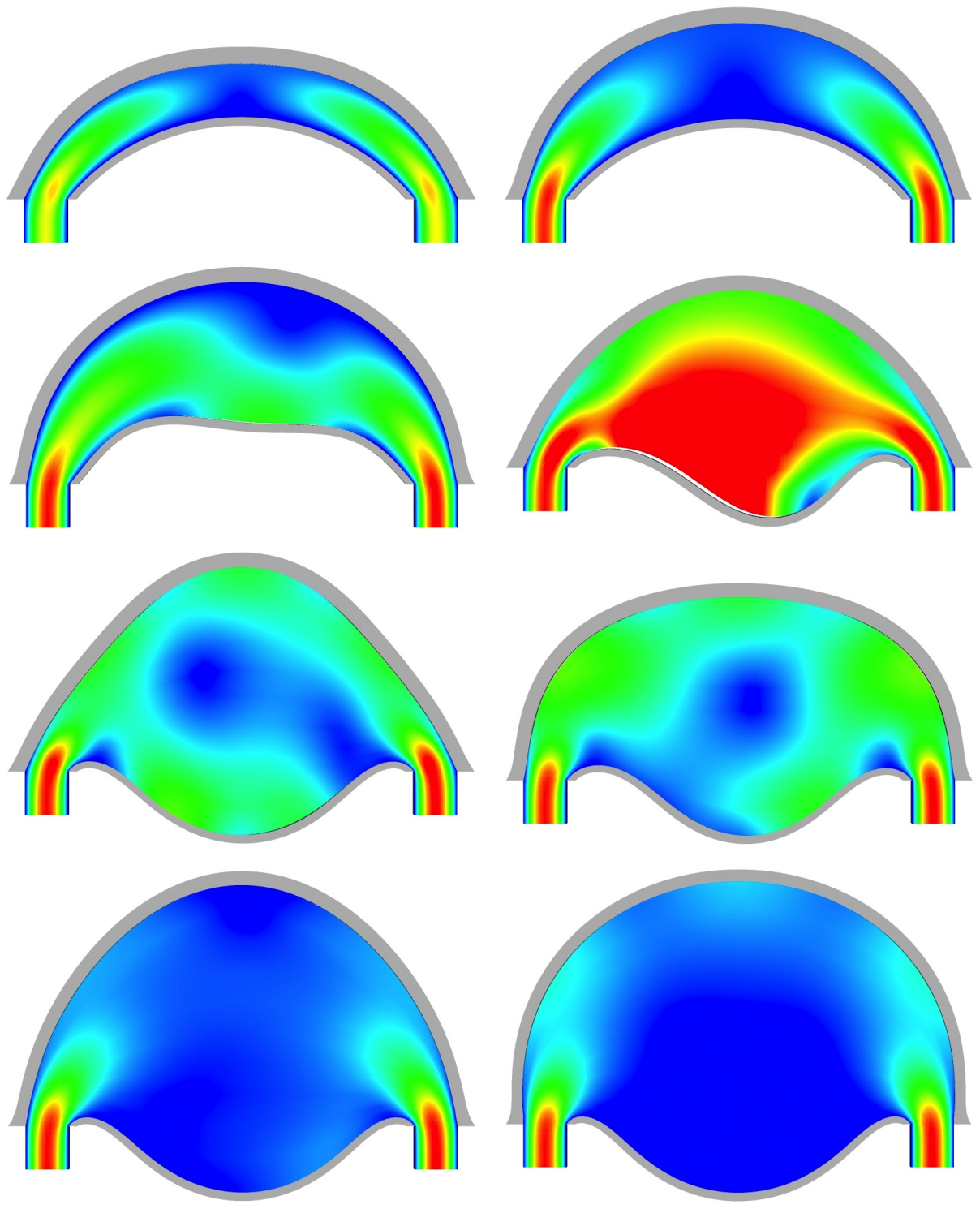

Figure 15: Snapshots of the fluid velocity and solid deformation at $t=$ $0.5,1.15,1.65,1.9,2.4,2.5,3.25,3.75$ (from left to right and top to bottom). Algorithm 3 with $r=1$ and $\tau=0.005$.

$E_{\mathrm{top}}=9 \cdot 10^{5}, E_{\mathrm{bottom}}=9 \cdot 10^{7}, \nu=0.3$ and $\alpha=\beta=0$. The simulations are performed in three-dimensions, by imposing symmetry conditions along the extrusion direction. The fluid and solid equations are discretized in space using continuous $\mathbb{P}_{1}$ finite elements (a SUPG/PSPG stabilized formulation is considered in the fluid).

It is well known that this type of problem cannot be solved via standard Dirichlet-Neumann partitioned procedures, since (at each iteration) the interface solid velocity does not necessarily satisfy the compatibility condition enforced by the incompressibility of the fluid (unless directly prescribed in the structure [23]). Algorithm 3 circumvents this issue in a natural fashion since the 
generalized Robin condition on the fluid removes the constraint on the interface solid velocity.

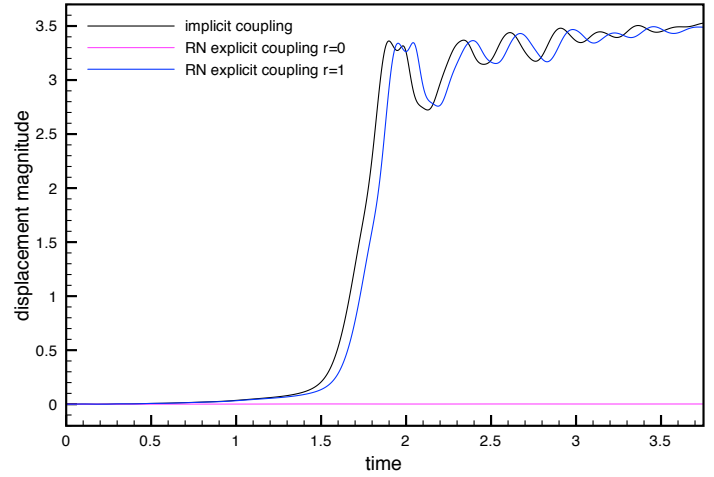

(a) $\tau=0.005$.

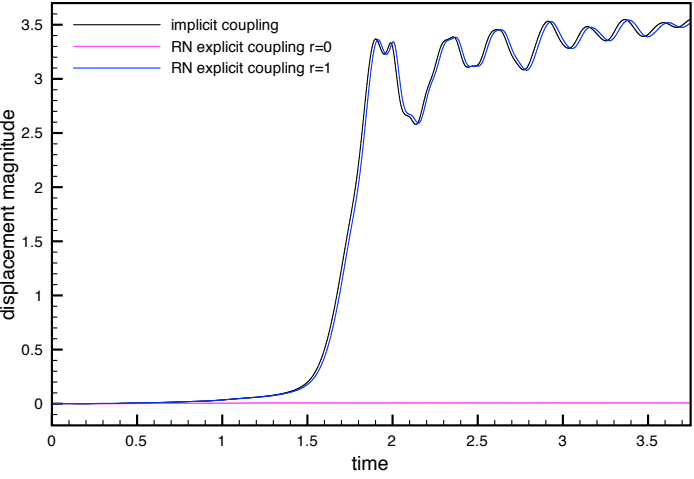

(b) $\tau=0.0025$.

Figure 16: Interface mid-point displacement magnitudes of the bottom structure obtained with the implicit coupling scheme and Algorithm 3.

Figure 16 shows the fluid velocity magnitude snapshots and the solid deformations at different time instants, obtained with Algorithm $3(r=1)$ and a time-step of $\tau=0.005$. As in [23, 13], the deformation is first mainly visible in the upper (more flexible) structure and then the lower structure buckles. In Figure 16, we have reported the interface mid-point displacement magnitude of the bottom structure obtained with Algorithm $3(r=0$ and $r=1)$ and the implicit coupling scheme, for $\tau=0.005$ and $\tau=0.0025$. The results of Algorithm 3 with $r=2$ are not reported in Figure 16 due to the lack of stability, smaller time-steps are required. The poor accuracy of the explicit coupling scheme without extrapolation $r=0$ is, once more, striking. The excess of massloss across the interface induced by the low-order perturbation of the kinematic coupling prevents the buckling of the bottom structure. On the contrary, the implicit scheme and Algorithm 3 with $r=1$ predict the collapse of the bottom structure for all the values of $\tau$ considered. The better accuracy of the implicit scheme with respect to Algorithm 3 with $r=1$ is visible for the largest time-step length.

\section{Conclusion}

In this paper we have proposed a generalization of the explicit Robin-Neumann schemes introduced in $[10,13]$ to the case of the coupling with thick-walled structures. The schemes are based on the following ingredients:

- Generalized notion of interface Robin consistency, using a mass-lumping approximation in the structure (Section 3.2);

- Implicit treatment of the sole interface solid inertia within the fluid;

- Appropriate extrapolations of the interface solid velocity and stress.

The second guarantees added-mass free stability (Theorem 4), while the third enables the staggered fluid-solid time-marching in a genuinely partitioned fashion.

Though the proposed extension retains the main stability properties of the original explicit Robin-Neumann schemes [13, 10], numerical evidence suggests that their optimal (first-order) 
accuracy is not necessarily preserved. Indeed, the order of the kinematic perturbation induced by the splitting is expected to be $\mathcal{O}\left((\tau / h)^{\frac{1}{2}}\right), \mathcal{O}\left(\tau / h^{\frac{1}{2}}\right)$ or $\mathcal{O}\left(\tau^{2} / h^{\frac{1}{2}}\right)$, depending on the order $r=0,1$ or 2 of the extrapolations. The factor $h^{-\frac{1}{2}}$ seems to be intrinsically related to the thin-walled character of the structure, through the non-uniformity of the discrete viscoelastic operator.

The comparison of the different methods has shown that the best robustness is obtained with the first-order extrapolation $(r=1)$. A salient feature of this scheme is that it simultaneously yields unconditional stability and convergence under a standard hyperbolic-CFL constraint. Furthermore, overall first-order accuracy is expected under a strengthened $3 / 2$-CFL constraint, $\tau=\mathcal{O}\left(h^{\frac{3}{2}}\right)$, without the need of correction iterations.

The schemes have been interpreted as a single iteration of a new, parameter free, RobinNeumann iterative procedure for the partitioned solution of implicit coupling. The convergence of this iterative method has been established (Theorem 5). Numerical evidence has confirmed that, unlike standard Robin-Neumann approaches, reducing the time-step length increases the convergence speed of the proposed iterations.

\section{References}

[1] S. Badia, F. Nobile, and C. Vergara. Fluid-structure partitioned procedures based on Robin transmission conditions. J. Comp. Phys., 227:7027-7051, 2008.

[2] Y. Bazilevs, V.M. Calo, T.J.R. Hughes, and Y. Zhang. Isogeometric fluid-structure interaction: theory, algorithms, and computations. Comput. Mech., 43(1):3-37, 2008.

[3] E. Burman and M.A. Fernández. Galerkin finite element methods with symmetric pressure stabilization for the transient Stokes equations: stability and convergence analysis. SIAM J. Numer. Anal., 47(1):409-439, 2008.

[4] E. Burman and M.A. Fernández. Stabilization of explicit coupling in fluid-structure interaction involving fluid incompressibility. Comput. Methods Appl. Mech. Engrg., 198(5-8):766$784,2009$.

[5] Erik Burman and Miguel Angel Fernández. Explicit strategies for incompressible fluidstructure interaction problems: Nitsche type mortaring versus Robin-Robin coupling. Research Report RR-8296, INRIA, May 2013.

[6] P. Causin, J.-F. Gerbeau, and F. Nobile. Added-mass effect in the design of partitioned algorithms for fluid-structure problems. Comput. Methods Appl. Mech. Engrg., 194(4244):4506-4527, 2005.

[7] P. Crosetto, S. Deparis, G. Fourestey, and A. Quarteroni. Parallel algorithms for fluidstructure interaction problems in haemodynamics. SIAM J. Sci. Comput., 33(4):1598-1622, 2011.

[8] C. Farhat, K. van der Zee, and Ph. Geuzaine. Provably second-order time-accurate looselycoupled solution algorithms for transient nonlinear aeroelasticity. Comput. Methods Appl. Mech. Engrg., 195(17-18):1973-2001, 2006.

[9] M.A. Fernández. Coupling schemes for incompressible fluid-structure interaction: implicit, semi-implicit and explicit. S $\overrightarrow{\mathrm{e}} M A$ J., (55):59-108, 2011. 
[10] M.A. Fernández. Incremental displacement-correction schemes for incompressible fluidstructure interaction: stability and convergence analysis. Numer. Math., 123(1):21-65, 2013.

[11] M.A. Fernández, J.F. Gerbeau, and C. Grandmont. A projection semi-implicit scheme for the coupling of an elastic structure with an incompressible fluid. Int. J. Num. Meth. Engrg., 69(4):794-821, 2007.

[12] M.A. Fernández and J. Mullaert. Displacement-velocity correction schemes for incompressible fluid-structure interaction. C. R. Math. Acad. Sci. Paris, 349(17-18):1011-1015, 2011.

[13] M.A. Fernández, J. Mullaert, and M. Vidrascu. Explicit Robin-Neumann schemes for the coupling of incompressible fluids with thin-walled structures. Comput. Methods Appl. Mech. Engrg. To appear.

[14] L. Formaggia, J.-F. Gerbeau, F. Nobile, and A. Quarteroni. On the coupling of 3D and 1D Navier-Stokes equations for flow problems in compliant vessels. Comp. Meth. Appl. Mech. Engrg., 191(6-7):561-582, 2001.

[15] L. Formaggia, A. Quarteroni, and A. Veneziani, editors. Cardiovascular Mathematics. Modeling and simulation of the circulatory system, volume 1 of Modeling, Simulation and Applications. Springer, 2009.

[16] C. Förster, W.A. Wall, and E. Ramm. Artificial added mass instabilities in sequential staggered coupling of nonlinear structures and incompressible viscous flows. Comput. Methods Appl. Mech. Engrg., 196(7):1278-1293, 2007.

[17] M.W. Gee, U. Küttler, and W. Wall. Truly monolithic algebraic multigrid for fluid-structure interaction. Int. J. Numer. Meth. Engng., 85(8):987-1016, 2011.

[18] G. Guidoboni, R. Glowinski, N. Cavallini, and S. Canic. Stable loosely-coupled-type algorithm for fluid-structure interaction in blood flow. J. Comp. Phys., 228(18):6916-6937, 2009 .

[19] F. Hecht. New development in FreeFem++. J. Numer. Math., 20(3-4):251-265, 2012.

[20] G. Hou, J. Wang, and A. Layton. Numerical methods for fluid-structure interaction - a review. Commun. Comput. Phys., 12(2):337-377, 2012.

[21] T.J.R. Hughes. The finite element method. Prentice Hall, 1987.

[22] U. Küttler and W.A. Wall. Fixed-point fluid-structure interaction solvers with dynamic relaxation. Comp. Mech., 43(1):61-72, 2008.

[23] U. Küttler, C. Förster, and W.A. Wall. A solution for the incompressibility dilemma in partitioned fluid-structure interaction with pure Dirichlet fluid domains. Comput. Mech., 38:417-429, 2006.

[24] M. Lukáčová, G. Rusnáková, and A. Hundertmark. Kinematic splitting algorithm for fluid-structure interaction in hemodynamics. Comput. Methods Appl. Mech. Engrg., 265(1):83-106, 2013.

[25] R.L. Muddle, M. Mihajlović, and M. Heil. An efficient preconditioner for monolithicallycoupled large-displacement fluid-structure interaction problems with pseudo-solid mesh updates. J. Comput. Phys., 231(21):7315-7334, 2012. 
[26] F. Nobile and C. Vergara. Partitioned Algorithms for Fluid-Structure Interaction Problems in Haemodynamics. Milan J. Math., 80(2):443-467, 2012.

[27] K.C. Park, C.A. Felippa, and J.A. Deruntz. Stabilization of staggered solution procedures for fluid-structure interaction analysis. In Belytschko. T. and T.L. Geers, editors, Computational Methods for Fluid-Structure Interaction Problems, volume 26, pages 95-124. American Society of Mechanical Engineers, 1977.

[28] S. Piperno. Explicit/implicit fluid/structure staggered procedures with a structural predictor and fluid subcycling for 2D inviscid aeroelastic simulations. Internat. J. Numer. Methods Fluids, 25(10):1207-1226, 1997.

[29] A. Quaini and A. Quarteroni. A semi-implicit approach for fluid-structure interaction based on an algebraic fractional step method. Math. Models Methods Appl. Sci., 17(6):957-983, 2007.

[30] V. Thomée. Galerkin finite element methods for parabolic problems. Springer, 2006.

[31] S. Turek and J. Hron. Proposal for numerical benchmarking of fluid-structure interaction between an elastic object and laminar incompressible flow. In Fluid-structure interaction, volume 53 of Lect. Notes Comput. Sci. Eng., pages 371-385. Springer, Berlin, 2006. 


\section{Contents}

1 Introduction $\quad 3$

2 A linear model problem $\quad 4$

3 Generalized Robin-Neumann methods 5

3.1 Space semi-discretization . . . . . . . . . . . . . . . . . . 5

3.2 Generalized interface Robin consistency . . . . . . . . . . . . . . . . . 7

3.3 Time discretization: explicit coupling schemes . . . . . . . . . . . . . . 8

3.4 Partitioned solution of implicit coupling . . . . . . . . . . . . . . . . 10

4 Numerical analysis $\quad 12$

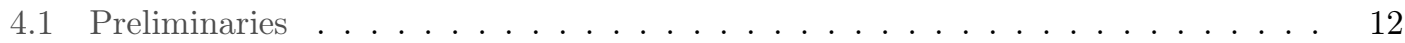

4.2 Stability analysis of the explicit coupling schemes . . . . . . . . . . . . . . 14

4.3 Convergence of the iterative solution procedure . . . . . . . . . . . . . . 17

5 The non-linear case $\quad 19$

5.1 The non-linear coupled problem . . . . . . . . . . . . . . . . . 19

5.2 Explicit coupling schemes . . . . . . . . . . . . . . . . . 19

6 Numerical experiments $\quad \mathbf{2 0}$

6.1 Numerical study in a two-dimensional test-case . . . . . . . . . . . . . . . . 20

6.1.1 Accuracy of the explicit coupled schemes . . . . . . . . . . . . . 21

6.1.2 Partitioned solution of implicit coupling . . . . . . . . . . . . . . 24

6.2 Pressure wave propagation in a straight tube . . . . . . . . . . . . . . 26

6.3 Flow around an elastic object . . . . . . . . . . . . . . . . . . . . . . . . . . . . . . . . . . .

6.4 Damped structural instability . . . . . . . . . . . . . . . . . . . . 29

7 Conclusion $\quad 31$ 


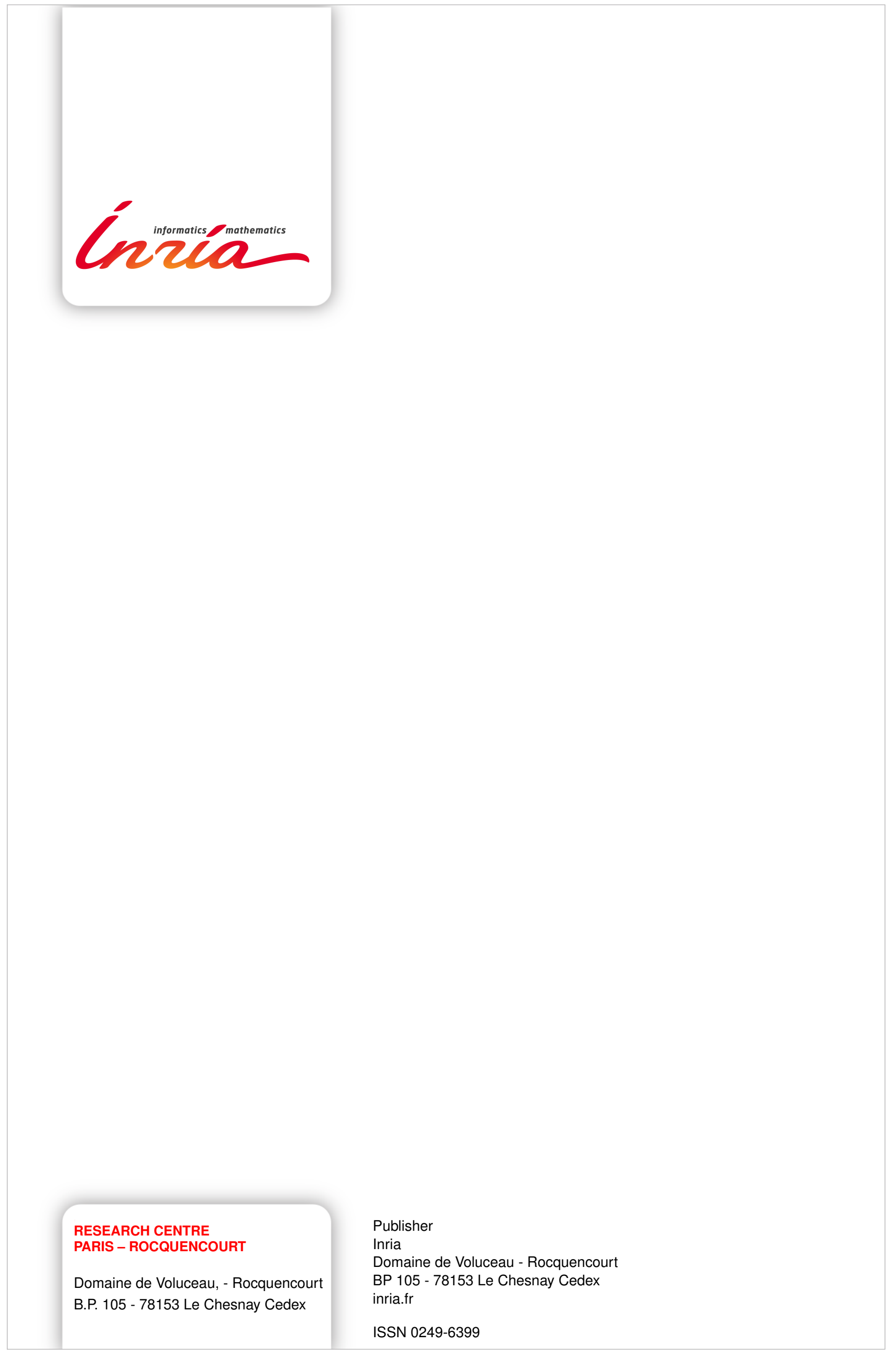

